\title{
Comprehensive Profiling and Characterization of The Absorbed Components and Metabolites in Mice Serum and Tissues Following Oral Administration of Qingfei Paidu Decoction by UHPLC-Q-Exactive-Orbitrap HRMS
}

\section{Wei Liu}

Key Laboratory of Liver and Kidney Diseases (Ministry of Education), Institute of Liver Diseases, Shanghai Key Laboratory of Traditional Chinese Clinical Medicine, Shuguang Hospital Affiliated to Shanghai University of Traditional Chinese Medicine

Jian Huang

Institute of Interdisciplinary Integrative Medicine Research, Shanghai University of Traditional Chinese Medicine

\section{Feng Zhang}

Institute of Interdisciplinary Integrative Medicine Research, Shanghai University of Traditional Chinese Medicine

\section{Congcong Zhang}

Institute of Chinese Materia Medica, Shanghai University of Traditional Chinese Medicine

\section{Rongsheng Li}

Key Laboratory of Liver and Kidney Diseases (Ministry of Education), Institute of Liver Diseases, Shanghai Key Laboratory of Traditional Chinese Clinical Medicine, Shuguang Hospital Affiliated to Shanghai University of Traditional Chinese Medicine

Yongli Wang

Institute of Chinese Materia Medica, Shanghai University of Traditional Chinese Medicine

Chaoran Wang

Dalian Institute of Chemical Physics, Chinese Academy of Sciences

Xinmiao Liang

Dalian Institute of Chemical Physics, Chinese Academy of Sciences

\section{Weidong Zhang}

Institute of Interdisciplinary Integrative Medicine Research, Shanghai University of Traditional Chinese Medicine

\section{Ling Yang}

Institute of Interdisciplinary Integrative Medicine Research, Shanghai University of Traditional Chinese Medicine

Ping Liu

Key Laboratory of Liver and Kidney Diseases (Ministry of Education), Institute of Liver Diseases, Shanghai Key Laboratory of Traditional Chinese Clinical Medicine, Shuguang Hospital Affiliated to Shanghai University of Traditional Chinese Medicine

Guang-Bo Ge ( $\sim$ geguangbo@dicp.ac.cn)

Institute of Interdisciplinary Integrative Medicine Research, Shanghai University of Triditional Chinese Medicine

\section{Research}

Keywords: Qingfei Paidu decoction (QFPDD), Corona virus disease 2019 (COVID-19), Chemical profiling, Absorbed components, High-Resolution Mass Spectrometry (HRMS)

Posted Date: December 30th, 2020

DOI: https://doi.org/10.21203/rs.3.rs-136211/v1

License: (a) (1) This work is licensed under a Creative Commons Attribution 4.0 International License. Read Full License 


\section{Abstract}

Background: Qingfei Paidu decoction (QFPDD) is a Chinese medicine compound formula recommended for combating corona virus disease 2019 (COVID-19) by National Health Commission of the People's Republic of China. This study aims to identify the main constituents in QFPDD and the absorbed components (including prototypes and metabolites) in serum and tissues after oral administration of QFPDD to mice.

Methods: A practical and sensitive method of UHPLC-Q-Exactive-Orbitrap HRMS was developed to identify the chemical constituents in QFPDD and the absorbed prototypes as well as the metabolites in mice serum and tissues following oral administration of QFPDD.

Results: A total of 405 chemicals, including 40 kinds of alkaloids, 162 kinds of flavonoids, 44 kinds of organic acids, 71 kinds of triterpene saponins and 88 kinds of other compounds in the water extract of QFPDD were tentatively identified via comparison with the retention times and MS/MS spectra of the standards or refereed by literature. With the help of the standards and in vitro metabolites, 195 chemical components (including 104 prototypes and 91 metabolites) were identified in mice serum after oral administration of QFPDD. In addition, 165, 177, 112, 120, 44, 53 constituents were identified in the lung, liver, heart, kidney, brain, and spleen of QFPDD-treated mice, respectively.

Conclusions: An UHPLC-Q-Orbitrap HRMS based method was established for chemical profiling the constituents in QFPDD, while the absorbed prototypes and metabolites occurring in mice serum and tissues were investigated following oral administration of QFPDD. These findings provided key information and guidance for further investigation on the pharmacologically active substances and clinical applications of QFPDD.

\section{Background}

Corona Virus Disease 2019 (COVID-19), a newly emerged infective disease, has spread all over the world, with a long incubation period, high infectivity and general susceptibility to all types of people [1-3]. The COVID-19 global pandemic has brought significantly negative effects for human health, economic and social stability. Researchers are now trying to find effective medications (including western therapeutics and herbal medicines) for combating COVID-19, while some therapeutics and herbal medicines have been used for treating COVID-19 in clinical settings [4-6]. In China, several Chinese medicine compound formulas, such as Qingfei Paidu decoction (QFPDD), Jingyin granules and Lianhua Qingwen capsule, have been validated playing an active role in combating this epidemic, especially alleviating the symptoms of some moderate and mild patients [7]. Among all recommend Chinese medicines for combating COVID19 in China, QFPDD has drawn much attention owing to its effectiveness of COVID-19. As the first Chinese medicine compound formula recommended by National Health Commission of the People's Republic of China for combating COVID-19, QFPDD has been used to treat thousands of COVID-19 patients with the total effective rate of $97 \%$ [8-10]. Following oral administration of QFPDD, the major symptoms and imaging manifestations of more than $60 \%$ patients were significantly improved, while the symptoms of $30 \%$ patients were stable and did not aggravate [11]. Early treatment with QFPDD is associated with favorable outcomes for patient recovery, viral shedding, hospital stay, and course of the disease. And early treatment with QFPDD may serve as an effective strategy in controlling the epidemic [12].

Unlike Western therapeutics, Chinese medicines are always the mixtures of a wide range of ingredients, these ingredients as well as their in vivo metabolites may interact with a wide range of targets in the human body in an extremely complex way $[13,14]$. To better understand the material basis for efficacy of QFPDD, the chemical constituents in this anti-COVID-19 Chinese medicine and the key components (including prototypes and metabolites) occurring in serum and target organs (such as the lung and the liver) following QFPDD treatment should be carefully investigated. Notably, QFPDD is deriving from four classic Chinese medicine prescriptions (including Maxing Shigan decoction, Shegan Mahuang decoction, Xiaochaihu decoction, and Wuling powder) that are used for treating epidemic diseases and the related inflammatory symptoms for thousands of years [15]. As a super combination of 20 herbs and a mineral drug (Gypsum Fibrosum), QFPDD is composed by hundreds of ingredients and parts of them may be absorbed in circulation system and then exert their bioactivites, such as anti-inflammatory and anticoagulation effects $[16,17]$. However, the chemical constituents in QFPDD and the absorbed components of this Chinese medicine have not been well-investigated yet, which strongly block further investigations on the pharmacological and toxicological studies, the quality control and clinical applications of this anti-COVID-19 Chinese medicine.

This study aims to investigate the main chemical constituents in QFPDD and the absorbed components (including prototypes and metabolites) in serum and tissues after oral administration of QFPDD to mice. To this end, an UHPLC-Q-Orbitrap HRMS based method was well-established for systematic profiling the constituents in QFPDD, while the absorbed components (including the prototypes and the metabolites) occurring in mouse serum and tissues were also identified via profiling the serum and tissue samples from QFPDD-treated mice. The prototype components in both QFPDD and mouse serum were tentatively identified via comparison with the retention times and MS/MS spectra of the standards or refereed by literature, while the in vivo metabolites were tentatively identified via comparison with the retention times and MS/MS spectra of the in vitro metabolites generated in mice liver microsomes (MLMs). Finally, a total of 405 chemicals (including 40 kinds of alkaloids, 162 kinds of flavonoids, 44 kinds of organic acids, 71 kinds of triterpene saponins and 88 kinds of other compounds) in the water extract of QFPDD were identified, while 195, 165, 177, 112, 120, 44, 53 constituents were identified in the serum, lung, liver, heart, kidney, brain, and spleen of QFPDD-treated mice, respectively. All these findings are very helpful for deep understanding the fates of the constituents in QFPDD, as well as the key information of the tissue distribution of the absorbed components of this anti-COVID-19 Chinese medicine.

\section{Materials And Exprimentals Chemicals and materials}

Herba Ephedrae (Mahuang, No. 191119-1), Ramulus Cinnamoni (Guizhi, No. 190802-1), Rhizoma Alismatis (Zixie, No. 191031-1), Polyporus (Zhuling, No. 191109-1), Flos Farfarae (Kuandonghua, No. 191207-1), Herba Asari (Xixin, No. 191220-1) and Rhizoma Dioscoreae (Shanyao, No. 191023-1) were purchased from Shanghai Wanshicheng Pharmaceutical Co., Ltd.(Shanghai, China). Gypsum Fibrosum (Shengshigao, No. 190801), Poria (Fuling, No. 191218), Radix 
Bupleuri (Chaihu, No. 200103), Rhizoma Pinelliae prepared with ginger juice (Jiangbanxia, No. 191009) and Herba Agastachis (Huoxiang, No. 190814) were purchased from Shanghai Hongqiao Traditional Chinese Medicine Decoction Piecess Co., Ltd. (Shanghai, China). Radix Glycyrrhizae Praeparata (Zhigancao, No. 191213), Rhizoma Belamcandae (Shegan, No. 191022), Fructus Aurantii Immaturus (Zhishi, No. 200119), Semen Armeniaceae Amarum (Xingren, No. 200108) were purchased from Shanghai Kangqiao Traditional Chinese Medicine Decoction Piecess Co., Ltd. (Shanghai, China). Radix Scutellariae (Huangqin, No. 19112307), Radix Asteris (Ziwan, No. 1909247) and Pericarpium Citri Reticulatae (Chenpi, No. 191119) were purchased from Shanghai Yanghetang Traditional Chinese Medicine Decoction Piecess Co., Ltd. (Shanghai, China). Rhizoma Atractylodis Macrocephalae (Baizhu, No. 190830) was purchased from Shanghai Qingpu Traditional Chinese Medicine Decoction Piecess Co., Ltd. (Shanghai, China)., Fresh Rhizoma Zingiberis Recens (Shengjiang) was purchased from the supermarket. All of the herbs were authenticated by prof. Changhong Wang, from the Institute of Chinese Materia Medica, Shanghai University of Traditional Chinese Medicine.

Arginine, uridine, uracil, tyrosine, leucine, isoleucine, adenine, 6-hydroxypurine, guanine, guanosine, adenosine, xanthosine,L-phenylalanine, tryptophan were purchased from Dalian Meilun Biotechnology Co. LTD. (Dalian, China). Neochlorogenic acid, caffeic acid, chlorogenic acid, cryptochlorogenic acid, Lamygdalin, prunasin, 1,3-O-dicaffeoylquinic acid, ferulic acid, isochlorogenic acid B, isochlorogenic acid A, resveratrol, isochlorogenic acid C, tilianin, 6Gingerol, 8-gingerol, 10-gingerol, alisol B-23-acetate and pachymic acid were purchased from Chengdu Biopurify Phytochemicals Ltd. (Chengdu, China). Quinic acid, stachydrine, citric acid, succinic acid, gallic acid, synephrine, phenylpropanolamine, D-demethyl pseudoephedrine, vanillic acid, ephedrine, pseudoephedrine, methylephedrine, catechin, epicatechin, neoliquiritin, 3,4-dihydroxybenzaldehyde, luteolin-7-O- $\beta$-D-glucoside, liquiritin, vitexin, tectoridin, isovitexin, hyperin, scutellarin, quercetin, isoquercitrin, verbascoside, hesperetin, isoaceteoside, hesperidin, astragalin, neohesperidin, cinnamaldehyde, liquiritigenin, isoliquiritin, ononin, naringin, luteolin, baicalin, cinnamic acid, naringenin, licochalcone A, licochalcone B, wogonoside, kaempferol, rosmarinic acid, isoliquiritigenin, baicalein, formononetin, sinensetin, irisflorentin, oroxylin A, nobiletin, atractylenolide-l, tangeretin, asarinin, glycyrrhizic acid, tussilagone and alisol A were provided by prof. Xinmiao Liang from Dalian Institute of Chemical Physics, Chinese Academy of Sciences (Dalian, China). The purities of those reference substances were more than $98 \%$.

Glucose 6-phosphate (G-6-P), glucose-6-phosphate dehydrogenase (G-6-PDH), nicotinamide adenine dinucleotide phosphate disodium salt ( $\beta$-NADP ${ }^{+}$), uridine di-phosphate glucuronic acid (UDPGA), alamethicin, S-adenosyl-L-methionine (SAM) and 3-phosphoadenosine 5-phosphosulfate (PAPS) were purchased from Sigma Aldrich Co. (St. Louis, MO, USA). Tris base and $\mathrm{MgCl}_{2}$ were purchased from Majorbio Biotech Corp., Ltd. (Shanghai, China). Pooled liver microsomes from male ICR/CD-1 mouse (MLM, Lot no. STOM) was ordered from Research Institute for Liver Diseases (RILD, Shanghai, China). Acetonitrile, methanol and formic acid of HPLC grade were purchased from Fisher Scientific Co. (Santa Clara, USA). Deionized water was produced with a Milli-Q Academic System (Millipore, Billerica, MA). All other reagents and solvents were either analytical or HPLC grade.

\section{Preparation of the Chinese multi-herbal formula QFPDD}

The Chinese multi-herbal formula Qingfei Paidu decoction (QFPDD) was prepared according to the following procedure, pieces of Herba Ephedrae (Mahuang) $45 \mathrm{~g}$, Radix Glycyrrhizae Praeparata (Zhigancao) $30 \mathrm{~g}$, Semen Armeniaceae Amarum (Xingren) $45 \mathrm{~g}$, Gypsum Fibrosum (Shengshigao) $75 \mathrm{~g}$ (fried first), Ramulus Cinnamoni (Guizhi) 45 g, Rhizoma Alismatis (Zixie) 45 g, Polyporus (Zhuling) 45 g, Rhizoma Atractylodis Macrocephalae (Baizhu) 45 g, Poria (Fuling) 75 g, Radix Bupleuri (Chaihu) 80 g, Radix Scutellariae (Huangqin) 30 g, Rhizoma Pinelliae prepared with ginger juice (Jiangbanxia) 45 g, Rhizoma Zingiberis Recens (Shengjiang) 45 g, Radix Asteris (Ziwan) 45 g, Flos Farfarae (Kuandonghua) 45 g, Rhizoma Belamcandae (Shegan) 45 g, Herba Asari (Xixin) $30 \mathrm{~g}$, Rhizoma Dioscoreae (Shanyao) $60 \mathrm{~g}$, Fructus Aurantii Immaturus (Zhishi) $30 \mathrm{~g}$, Pericarpium Citri Reticulatae (Chenpi) $30 \mathrm{~g}$ and Herba Agastachis (Huoxiang) $45 \mathrm{~g}$, were mixed together and macerated in $12 \mathrm{~L}$ deionized water for $40 \mathrm{~min}$ before being decocted. After then, $980 \mathrm{~g}$ of the herbal mixture was decocted for $45 \mathrm{~min}$. A total of 100 batches of QFPDD were decocted repeatly and then combined. After filtration, the decoction ( $180 \mathrm{~L})$ was further concentrated to $20 \mathrm{~L}$ by rotary evaporation at $40-50{ }^{\circ} \mathrm{C}$ in vacuum with a $93.98 \%$ concentrated yield. Finally, $6.52 \mathrm{~kg}$ powder was obtained by spray-drying with a $78.55 \%$ spray-dried yield.

\section{Preparation of QFPDD extract}

QFPDD $(1.0 \mathrm{~g})$ was weighed and dissolved in $125 \mathrm{~mL}$ deionized water by ultrasonic $(250 \mathrm{~W}, 50 \mathrm{HZ})$ for $1 \mathrm{~h}$. Then, the sample solution was centrifuged at 10000 $\times \mathrm{g}$ for $15 \mathrm{~min}$. The supernatant solution was filtered through a $0.22 \mu \mathrm{m}$ membrane for further analysis.

\section{Animal handling and sampling}

Twelve male C57BL/6J mice (SPF grade, weight of $20 \pm 2 \mathrm{~g}$ ) were purchased from Shanghai Slac Laboratory Animal Co. Ltd. (Shanghai, China) and were fed in Experimental Animal Center of Shanghai University of Traditional Chinese Medicine. All of animals were housed in an environment-controlled breeding room at $25^{\circ} \mathrm{C}$ with a 12 hours dark/light cycle. All experiments performed in mice were in accordance with the State Committee of Science and Technology of P.R. of China, and the protocol for this study was approved and monitored by the Animal Ethics Committee of Shanghai University of Traditional Chinese Medicine (PZSHUTCM200717018).

After seven days of acclimatization, twelve male mice were randomly divided into two groups (6 mice for each group). QFPDD group was oral administered QFPDD (18 g dried material $/ \mathrm{kg}$ ); control group was oral administered deionized water. All animals were fasted overnight before the experiments and had free access to water. One hour after the once oral administration, all mice were anesthetized, blood samples were collected from the abdominal aorta, and their various tissues (lung, liver, heart, kidney, brain, spleen) were removed rapidly. Blood samples were placed at room temperature for $1 \mathrm{~h}$ until solidifcation. Then, serum samples were obtained by centrifuged at $5000 \mathrm{rpm}$ for $15 \mathrm{~min}$ at $4{ }^{\circ} \mathrm{C}$. All samples were stored at $-80^{\circ} \mathrm{C}$ until analysis. The serum samples ( $\left.200 \mu \mathrm{L}\right)$ were added five times methanol $(1 \mathrm{~mL}$ ) was to the serum samples, vortexed and then, centrifuged at $10000 \times \mathrm{g}$ for $15 \mathrm{~min}$. The supernatant ( $960 \mu \mathrm{L})$ was dried with nitrogen gas. The residue was redissolved in $80 \mu \mathrm{L} \mathrm{10 \%} \mathrm{methanol,} \mathrm{vortexed} \mathrm{and} \mathrm{then,} \mathrm{centrifuged} \mathrm{at} 10000 \times \mathrm{g}$ for $15 \mathrm{~min}$, and the filtrate was used as the LC/MS sample. The $100 \mathrm{mg}$ various tissues (lung, liver, heart, kidney, brain, spleen) were homogenized in $1 \mathrm{~mL}$ methanol on ice, the homogenate of them was centrifuged at $4{ }^{\circ} \mathrm{C}$ at $10000 \times \mathrm{g}$ for $15 \mathrm{~min}$, The supernatant $(800 \mu \mathrm{L})$ was dried with nitrogen gas. The residue was redissolved in $80 \mu \mathrm{L} 10 \%$ methanol, 
vortexed and then, centrifuged at $10000 \times \mathrm{g}$ for $15 \mathrm{~min}$, and the filtrate was used as the LC/MS sample. $2 \mu \mathrm{L}$ aliquot was injected for UHPLC-Q-Exactive Orbitrap HRMS analysis.

In vitro metabolism of the major constituents isolated from QFPDD in MLMs

Microsomal incubation with NADPH: The incubation was performed in a $50 \mathrm{mM}$ Tris- $\mathrm{HCl}$ buffer (pH 7.4) containing MLMs (1.0 mg protein/mL), the NADPHgenerating system contained $10 \mathrm{mM} \mathrm{G-6-P,} 1 \mathrm{mM} \mathrm{NADP}+, 4 \mathrm{mM} \mathrm{MgCl}_{2}$, and 1 unit/mL G-6-PDH, and $200 \mu \mathrm{M}$ analyte (nobiletin) which was previously dissolved in methanol with concentration $200 \mu \mathrm{M}$ in a total volume of $200 \mu \mathrm{L}[18,19]$. The reactions were terminated with $600 \mu \mathrm{L}$ ice-cold acetonitrile after $60 \mathrm{~min}$ incubation. The mixture was centrifuged at $10,000 \times \mathrm{g}$ for $15 \mathrm{~min}$, and then the supernatant $(720 \mu \mathrm{L})$ was evaporated to dryness by a gentle stream of nitrogen $\left(37^{\circ} \mathrm{C}\right)$. The residue was dissolved by $90 \mu \mathrm{L}$ of $5 \%$ methanol and centrifuged at $10,000 \times \mathrm{g}$ for 10 min. $2 \mu \mathrm{L}$ of the supernatant was injected for UHPLC-Q-Exactive Orbitrap HRMS system. Control samples containing no NADPH or substrates were prepared. Incubation was performed in duplicate.

Microsomal incubation with NADPH and UDPGA: $200 \mu \mathrm{M}$ analyte (baicalein, scutellarin, wogonin, norwogonin, formononetin, liquiritigenin, hesperetin, naringenin, quercetin, oroxylin A and caffeic acid, each compound was incubated separately ) was mixed with alamethicin ( $25 \mu \mathrm{g} / \mathrm{mg}$ protein) and the microsomes (1.0 mg protein/mL) in $100 \mathrm{mM}$ Tris- $\mathrm{HCl}$ buffer $(50 \mathrm{mM}, \mathrm{pH} 7.4)$. After 5 min of pre-incubation at $37^{\circ} \mathrm{C}$, the incubation reaction was initiated by the addition of G-6-P (10 mM), G-6-PDH (1 unit/mL), NADP ${ }^{+}(1.0 \mathrm{mM})$, and UDPGA (5.0 mM). The total incubation volume was $200 \mu \mathrm{L}$. The reactions were terminated with $600 \mu \mathrm{L}$ ice-cold acetonitrile after $1 \mathrm{~h}$ of incubation. The mixture was centrifuged at $10,000 \times g$ for 15 min, and then the supernatant $(720 \mu \mathrm{L})$ was evaporated to dryness by a gentle stream of nitrogen at $37^{\circ} \mathrm{C}$. The residue was dissolved by $90 \mu \mathrm{L}$ of $5 \%$ methanol and centrifuged at $10,000 \times g$ for $15 \mathrm{~min}$. The supernatant $(2 \mu \mathrm{L})$ was then injected for UHPLC-Q-Exactive Orbitrap HRMS system. Control samples without NADPH and UDPGA or substrates were prepared. Incubation was performed in duplicate.

Microsomal incubation with NADPH and PAPS: The incubation was performed in a $50 \mathrm{mM}$ Tris- $\mathrm{HCl}$ buffer at pH 7.4 containing microsomes (1.0 mg protein/mL), the NADPH-generating system contained $10 \mathrm{mM} \mathrm{G-6-P,} 1 \mathrm{mM} \mathrm{NADP}+, 4 \mathrm{mM} \mathrm{MgCl} 2$, and 1 unit/mL of G-6-PDH, $100 \mu \mathrm{M}$ PAPS, and $200 \mu \mathrm{M}$ analyte (baicalein, wogonin, formononetin, liquiritigenin, hesperetin and caffeic acid, each compound was incubated separately) in a total volume of $200 \mu \mathrm{L}$. The reactions were terminated with $600 \mu \mathrm{L}$ ice-cold acetonitrile after $60 \mathrm{~min}$ incubation. The next process was as same as above and the control samples containing no NADPH, no PAPS or substrates were prepared. Each of the incubations was performed in duplicate.

Microsoma incubation with NADPH and SAM: The incubation was performed in a $50 \mathrm{mM}$ Tris- $\mathrm{HCl}$ buffer at pH 7.4 containing microsomes (1.0 mg protein/mL), the NADPH-generating system contained $10 \mathrm{mM} \mathrm{G}-6-\mathrm{P}, 1 \mathrm{mM} \mathrm{NADP}{ }^{+}, 4 \mathrm{mM} \mathrm{MgCl}{ }_{2}$, and 1 unit/mL of G-6-PDH, $100 \mu \mathrm{M} \mathrm{SAM}$, and $200 \mu \mathrm{M}$ analyte (baicalein, scutellarin, chlorogenic acid and caffeic acid, each compound was incubated separately) in a total volume of $200 \mu \mathrm{L}$. The reactions were terminated with $600 \mu \mathrm{L}$ ice-cold acetonitrile after 60 min incubation. The next process was as same as above and the control samples containing no NADPH, no SAM or substrates were prepared. Each of the incubations was performed in duplicate.

\section{Chromatography conditions for UHPLC-Q-Exactive Orbitrap HRMS}

Chromatographic separation was performed on a UHPLC-Q-Exactive Orbitrap system (Thermo Fisher Scientific Inc., Grand Island, NY, USA). The UHPLC system consisted of a Thermo Scientific Dionex Ultimate 3000 Series RS pump coupled with a Thermo Scientific Dionex Ultimate 3000 Series TCC-3000RS column compartments and WPS-3000 autosampler controlled by Chromeleon 7.2 Software. The cooling autosampler was set at $4{ }^{\circ} \mathrm{C}$ and protected from light, and the column heater was set at $40^{\circ} \mathrm{C}$. A Waters ACQUITY UPLC BEH C18 column $(2.1 \times 100 \mathrm{~mm}, 1.7 \mu \mathrm{m})$ was employed with the temperature set at $40{ }^{\circ} \mathrm{C}$. The mobile phase consisted of $A$ (methanol) and $B\left(0.1 \%\right.$ formic acid) at a flow rate of $0.3 \mathrm{~mL} \cdot \mathrm{min}^{-1}$ and eluted with gradient elution: $0-4 \mathrm{~min}(4 \% \mathrm{~A})$, 4-10 min $(4 \%-12 \% A), 10-30 \min (12 \%-70 \% A), 30-35 \min (70 \% A), 35-38 \min (70-95 \% A), 38-42 \min (95 \%$ A), $42-45$ min $(4 \% A)$. The injection volume was $2 \mu \mathrm{L}$.

The mass spectrometer Q-Exactive Orbitrap system was connected to the UHPLC system via heated electrospray ionization and controlled by Xcalibur 4.1 software that was used for data collection and analysis. The electrospray ionization source was operated and optimized in negative and positive ionization mode. The optimized parameters of mass spectrometry were: capillary temperature: $325^{\circ} \mathrm{C}$; sheath gas $\left(\mathrm{N}_{2}\right)$ flow rate: 45 arbitrary units; auxiliary gas $\left(\mathrm{N}_{2}\right)$ flow rate: 8 arbitrary units; sweep gas flow rate: 0 arbitrary units; spray voltage: 2.5 kV (negative), 3.5 kV (positive); S-lens RF level: $50 \mathrm{~V}$; auxilliary gas heater temperature, $300^{\circ} \mathrm{C}$; scan mode: Full MS/SIM and Full MS/dd-MS2 mode, which includes 1 first-level full scan (resolution 70000 FWHM) and 1 datadependent secondary scan (resolution $17500 \mathrm{FWHM}$ ) 2 events, the scanning range is $80-1200 \mathrm{~m} / \mathrm{z}$, and the collision energy gradient is $20 \mathrm{~V}$, $50 \mathrm{~V}$, $100 \mathrm{~V}$.

\section{Establishment of QFPDD chemical database and chemical profiling}

By searching databases, such as PubMed of the US National Library Medicine and the National Institutes of Health, SciFinder Scholar of American Chemical Society and the Chinese National Knowledge Infrastructure (CNKI) of Tsinghua University, the Encyclopedia of Traditional Chinese Medicine Database (ETCM, http://www.tcmip.cn/ETCM/index.php/Home/Index/index.html), Traditional Chinese Medicine Integrated Database (TCMID,

http://www.megabionet.org/tcmid/), and all components reported in the literature on 21 herbs of QFPDD were summarized in a TraceFinder software (version 3.3) to establish a database, which includes the name, molecular formula, chemical structure and literature of each published known compound. After the sample was injected, the quasi-molecular ion information was obtained, and the molecular formula was fitted by Xcalibar 3.0 software, and matched with the $\mathrm{CD}$ (Compound discovery, 2.1) compound analysis and identification software and self-built database, and the possible chemical composition was inferred. Combined with the relative retention time and fragment ion information of reference substances and literature, the chemical composition was identified.

\section{Results}

\section{Identification and characterization of chemical constituents from QFPDD}


Under the optimal conditions described above, the constituents in the crude extract of QFPDD were comprehensively analyzed by UHPLC-Q-Exactive Orbitrap HRMS, with a running time of $45 \mathrm{~min}$. The total ion chromatograms (TICs) of the extract of QFPDD under both positive and negative ion modes are shown in Fig. 1. The protonated molecular weights of all identified compounds were calculated within an error of 10 ppm. Following carefully comparison with the retention times and MS/MS spectra of the reference standards, reference literature, Chemical Book and self-bulit databases, a total of 405 chemicals were identified or tentatively characterized from QFPDD, including 40 kinds of alkaloids, 162 kinds of flavonoids, 44 kinds of organic acids, 71 kinds of triterpene saponins and 88 kinds of other compounds (Table 1). The detailed information of the identified chemical constituents in QFPDD including retention time, precise molecular weight, MS/MS fragment ions and the source of each constituent, have been listed in Table 1. Among all identified chemical constituents, ninety-one constituents are confirmed by the authentic standards. 
Table 1

Ingredient identification of QFPDD by UHPLC-Q-Exactive Orbitrap HRMS

\begin{tabular}{|c|c|c|c|c|c|c|c|c|}
\hline No. & $\begin{array}{l}\mathrm{RT} \\
/ \mathrm{min}\end{array}$ & $\begin{array}{l}\text { lon } \\
\text { model }\end{array}$ & $\begin{array}{l}\text { Measured } \\
\text { mass /Da }\end{array}$ & $\begin{array}{l}\text { Calculated } \\
\text { mass /Da }\end{array}$ & $\begin{array}{l}\text { Error } \\
\text { /ppm }\end{array}$ & $\begin{array}{l}\text { Molecular } \\
\text { formula }\end{array}$ & Identification & Fragment ions \\
\hline $1^{*}$ & 0.85 & {$[\mathrm{M}-\mathrm{H}]^{-}$} & 173.1035 & 173.1033 & 1.085 & $\mathrm{C}_{6} \mathrm{H}_{14} \mathrm{~N}_{4} \mathrm{O}_{2}$ & Arginine & $131.0825 ; 96.9672$ \\
\hline 2 & 0.88 & $\begin{array}{l}{[\mathrm{M}+} \\
\mathrm{FA}-\mathrm{H}]^{-}\end{array}$ & 195.0502 & 195.0499 & 1.491 & $\mathrm{C}_{5} \mathrm{H}_{10} \mathrm{O}_{5}$ & Arabinose & $\begin{array}{l}\text { 195.0561; } 179.0589 ; 165.04 \\
\text { 151.0635; } 135.0313 ; 75.009 \\
\text { 71.0117; }\end{array}$ \\
\hline 3 & 0.89 & $\begin{array}{l}{[\mathrm{M}+} \\
\mathrm{H}]^{+}\end{array}$ & 118.0865 & 118.0863 & 0.205 & $\mathrm{C}_{5} \mathrm{H}_{11} \mathrm{NO}_{2}$ & Betaine & 58.0655 \\
\hline 4 & 0.90 & {$[\mathrm{M}-\mathrm{H}]^{-}$} & 149.0080 & 149.0081 & -0.084 & $\mathrm{C}_{4} \mathrm{H}_{6} \mathrm{O}_{6}$ & Tartaric acid & $\begin{array}{l}149.0092 ; 87.0087 ; 72.9927 \\
59.0134\end{array}$ \\
\hline 5 & 0.90 & {$[\mathrm{M}-\mathrm{H}]^{-}$} & 157.0356 & 157.0356 & -0.017 & $\mathrm{C}_{4} \mathrm{H}_{6} \mathrm{O}_{3} \mathrm{~N}_{4}$ & Allantoin & 59.03; 97.00; 71.03; \\
\hline 6 & 0.90 & {$[\mathrm{M}-\mathrm{H}]^{-}$} & 179.0552 & 179.0550 & 0.980 & $\mathrm{C}_{6} \mathrm{H}_{12} \mathrm{O}_{6}$ & Glucose & $\begin{array}{l}\text { 179.0570; } 161.0461 ; 87.009 \\
71.0137\end{array}$ \\
\hline 7 & 0.91 & $\begin{array}{l}{[\mathrm{M}+} \\
\mathrm{H}]^{+}\end{array}$ & 138.0549 & 138.0550 & -0.085 & $\mathrm{C}_{7} \mathrm{H}_{7} \mathrm{NO}_{2}$ & Trigonelline & $92.05 ; 78.03 ; 65.04 ; 51.02$ \\
\hline $8^{*}$ & 0.92 & {$[\mathrm{M}-\mathrm{H}]^{-}$} & 191.0553 & 191.0550 & 1.389 & $\mathrm{C}_{7} \mathrm{H}_{12} \mathrm{O}_{6}$ & Quinic acid & $191.0563 ; 85.0297$ \\
\hline $9 *$ & 0.95 & $\begin{array}{l}{[\mathrm{M}+} \\
\mathrm{H}]^{+}\end{array}$ & 144.1018 & 144.1019 & -0.125 & $\mathrm{C}_{7} \mathrm{H}_{13} \mathrm{NO}_{2}$ & Stachydrine & $144.1022 ; 84.0807 ; 56.0491$ \\
\hline 10 & 0.96 & {$[\mathrm{M}-\mathrm{H}]^{-}$} & 207.0141 & 207.0135 & 2.543 & $\mathrm{C}_{6} \mathrm{H}_{8} \mathrm{O}_{8}$ & Hydroxycitric acid & $\begin{array}{l}207.0082 ; 189.0049 ; 127.00 \\
83.0146\end{array}$ \\
\hline 11 & 0.98 & {$[\mathrm{M}-\mathrm{H}]^{-}$} & 341.1091 & 341.1078 & 3.788 & $\mathrm{C}_{12} \mathrm{H}_{22} \mathrm{O}_{11}$ & Sucrose & $\begin{array}{l}179.0563 ; 161.0456 ; 119.03 \\
89.0249\end{array}$ \\
\hline 12 & 1.02 & $\begin{array}{l}{[\mathrm{M}+} \\
\mathrm{FA}-\mathrm{H}]^{-}\end{array}$ & 549.1677 & 549.1661 & 2.767 & $\mathrm{C}_{18} \mathrm{H}_{32} \mathrm{O}_{16}$ & Raffinose & $503.1624 ; 221.0672 ; 179.05$ \\
\hline 13 & 1.05 & {$[\mathrm{M}-\mathrm{H}]^{-}$} & 115.0023 & 115.0026 & -2.479 & $\mathrm{C}_{4} \mathrm{H}_{4} \mathrm{O}_{4}$ & Maleic acid & 71.0142 \\
\hline 14 & 1.05 & {$[\mathrm{M}-\mathrm{H}]^{-}$} & 133.0129 & 133.0131 & -0.230 & $\mathrm{C}_{4} \mathrm{H}_{6} \mathrm{O}_{5}$ & Malic acid & $115.0054 ; 71.0150$ \\
\hline 15 & 1.06 & $\begin{array}{l}{[\mathrm{M}+} \\
\left.\mathrm{NH}_{4}\right]^{+}\end{array}$ & 136.0617 & 136.0604 & 1.276 & $\mathrm{C}_{4} \mathrm{H}_{6} \mathrm{O}_{4}$ & Succinic acid & $\begin{array}{l}136.0609 ; 92.0239 ; 72.9924 \\
65.0131\end{array}$ \\
\hline 16 & 1.13 & $\begin{array}{l}{[\mathrm{M}+} \\
\mathrm{FA}-\mathrm{H}]^{-}\end{array}$ & 711.2212 & 711.2190 & 3.130 & $\mathrm{C}_{24} \mathrm{H}_{42} \mathrm{O}_{21}$ & Maltotetraose isomer & $\begin{array}{l}665.2108 ; 485.1475 ; 443.14 \\
383.1172 ; 341.1066\end{array}$ \\
\hline 17 & 1.14 & $\begin{array}{l}{[\mathrm{M}+} \\
\mathrm{H}]^{+}\end{array}$ & 118.0864 & 118.0863 & 0.175 & $\mathrm{C}_{5} \mathrm{H}_{11} \mathrm{NO}_{2}$ & Valine & $72.0805 ; 55.0539$ \\
\hline 18 & 1.27 & $\begin{array}{l}{[\mathrm{M}+} \\
\mathrm{Na}]^{+}\end{array}$ & 851.2632 & 851.2639 & -0.778 & $\mathrm{C}_{30} \mathrm{H}_{52} \mathrm{O}_{26}$ & Pentaose & $851.2523 ; 527.1444$ \\
\hline 19 & 1.28 & {$[\mathrm{M}-\mathrm{H}]^{-}$} & 827.2685 & 827.2663 & 2.638 & $\mathrm{C}_{30} \mathrm{H}_{52} \mathrm{O}_{26}$ & Maltopentaose & $\begin{array}{l}647.2048 ; 503.1619 ; 341.10 \\
323.1004 ; 179.0565 ; 161.04\end{array}$ \\
\hline 20 & 1.31 & $\begin{array}{l}{[\mathrm{M}+} \\
\mathrm{H}]^{+}\end{array}$ & 123.0554 & 123.0553 & 0.131 & $\mathrm{C}_{6} \mathrm{H}_{6} \mathrm{~N}_{2} \mathrm{O}$ & Nicotinamide & $78.03 ; 63.01 ; 53.04$ \\
\hline $21^{\star}$ & 1.34 & {$[\mathrm{M}-\mathrm{H}]^{-}$} & 191.0183 & 191.0186 & 1.052 & $\mathrm{C}_{6} \mathrm{H}_{8} \mathrm{O}_{7}$ & Citric acid & 191.0203; 111.0100; 87.009 \\
\hline 22 & 1.39 & $\begin{array}{l}{[\mathrm{M}+} \\
\mathrm{H}]^{+}\end{array}$ & 127.0390 & 127.0390 & 0.049 & $\mathrm{C}_{6} \mathrm{H}_{6} \mathrm{O}_{3}$ & 5-Hydroxymethylfurfural & $\begin{array}{l}\text { 127.0384; } 109.0276 ; 81.033 \\
68.9974\end{array}$ \\
\hline 23 & 1.42 & {$[\mathrm{M}-\mathrm{H}]^{-}$} & 1151.3737 & 1151.3720 & 1.481 & $\mathrm{C}_{42} \mathrm{H}_{72} \mathrm{O}_{36}$ & Fructo-oligosaccharide Dp7/Gf6 & $\begin{array}{l}\text { 1151.3765; } 1031.3220 ; 989 . \\
827.2672\end{array}$ \\
\hline 24 & 1.44 & {$[\mathrm{M}-\mathrm{H}]^{-}$} & 827.2687 & 827.2663 & 2.868 & $\mathrm{C}_{30} \mathrm{H}_{52} \mathrm{O}_{26}$ & Fructo-oligosaccharide Dp5/Gf4 & $827.2575 ; 503.1631 ; 179.05$ \\
\hline 25 & 1.46 & {$[\mathrm{M}-\mathrm{H}]^{-}$} & 989.3212 & 989.3191 & 2.122 & $\mathrm{C}_{36} \mathrm{H}_{62} \mathrm{O}_{31}$ & $\begin{array}{l}\text { Maltohexaose,fructo-oligosaccharide } \\
\text { Dp6/Gf5 }\end{array}$ & $\begin{array}{l}989.3319 ; 827.2717 ; 665.21 \\
503.1650 ; 341.1082\end{array}$ \\
\hline 26 & 1.48 & $\begin{array}{l}{[\mathrm{M}+} \\
\mathrm{H}]^{+}\end{array}$ & 130.0499 & 130.0499 & 0.010 & $\mathrm{C}_{5} \mathrm{H}_{7} \mathrm{NO}_{3}$ & Pyroglutamic acid & $84.0441 ; 56.0490$ \\
\hline 27 & 1.55 & $\begin{array}{l}{[\mathrm{M}+} \\
\mathrm{FA}-\mathrm{H}]^{-}\end{array}$ & 711.2216 & 711.2190 & 3.650 & $\mathrm{C}_{24} \mathrm{H}_{42} \mathrm{O}_{21}$ & Maltotetraose & $\begin{array}{l}665.2161 ; 545.1857 ; 341.10 \\
179.0555 ; 89.0249\end{array}$ \\
\hline
\end{tabular}




\begin{tabular}{|c|c|c|c|c|c|c|c|c|}
\hline No. & $\begin{array}{l}\mathrm{RT} \\
/ \mathrm{min}\end{array}$ & $\begin{array}{l}\text { lon } \\
\text { model }\end{array}$ & $\begin{array}{l}\text { Measured } \\
\text { mass /Da }\end{array}$ & $\begin{array}{l}\text { Calculated } \\
\text { mass } / \mathrm{Da}\end{array}$ & $\begin{array}{l}\text { Error } \\
\text { /ppm }\end{array}$ & $\begin{array}{l}\text { Molecular } \\
\text { formula }\end{array}$ & Identification & Fragment ions \\
\hline $28^{*}$ & 1.57 & {$[\mathrm{M}-\mathrm{H}]^{-}$} & 243.0621 & 243.0612 & 3.692 & $\mathrm{C}_{9} \mathrm{H}_{12} \mathrm{~N}_{2} \mathrm{O}_{6}$ & Uridine & $128.0393 ; 110.0238$ \\
\hline $29 *$ & 1.58 & $\begin{array}{l}{[\mathrm{M}+} \\
\mathrm{H}]^{+}\end{array}$ & 113.0348 & 113.0346 & 2.177 & $\mathrm{C}_{4} \mathrm{H}_{4} \mathrm{~N}_{2} \mathrm{O}_{2}$ & Uracil & $\begin{array}{l}113.0369 ; 96.0086 ; 80.9743 \\
68.0126 ; 52.0186\end{array}$ \\
\hline 30 & 1.61 & $\begin{array}{l}{[\mathrm{M}+} \\
\mathrm{FA}-\mathrm{H}]^{-}\end{array}$ & 549.1677 & 549.1661 & 2.876 & $\mathrm{C}_{18} \mathrm{H}_{32} \mathrm{O}_{16}$ & Melezitose & $\begin{array}{l}503.1603 ; 323.0967 ; 179.05 \\
89.0243\end{array}$ \\
\hline $31 *$ & 1.64 & $\begin{array}{l}{[\mathrm{M}+} \\
\mathrm{H}]^{+}\end{array}$ & 182.0811 & 182.0812 & -0.438 & $\mathrm{C}_{9} \mathrm{H}_{11} \mathrm{NO}_{3}$ & Tyrosine & $\begin{array}{l}134.06 ; 107.05 ; 91.05 ; 77.04 \\
65.04\end{array}$ \\
\hline $32^{*}$ & 1.72 & {$[\mathrm{M}-\mathrm{H}]^{-}$} & 117.0197 & 117.0182 & -2.266 & $\mathrm{C}_{4} \mathrm{H}_{6} \mathrm{O}_{4}$ & Succinic acid & $73.0294 ; 55.0216$ \\
\hline 33 & 1.79 & $\stackrel{[\mathrm{M}+}{\mathrm{Na}]+}$ & 289.1391 & 289.1410 & -6.780 & $\mathrm{C}_{15} \mathrm{H}_{22} \mathrm{O}_{4}$ & 4-Gingerol & $\begin{array}{l}\text { 177.1019; } 163.0854 ; 145.07 \\
133.0757\end{array}$ \\
\hline 34 & 1.8 & {$[\mathrm{M}-\mathrm{H}]-$} & 115.0023 & 115.0026 & -2.218 & $\mathrm{C}_{4} \mathrm{H}_{4} \mathrm{O}_{4}$ & Fumaric acid or its isomers & $\begin{array}{l}\text { 115.004; } 99.9255 ; 83.9301 \\
\text { 53.0040 }\end{array}$ \\
\hline 35 & 1.85 & $\begin{array}{l}{[\mathrm{M}+} \\
\left.\mathrm{NH}_{4}\right]^{+}\end{array}$ & 194.1175 & 194.1176 & -0.079 & $\mathrm{C}_{11} \mathrm{H}_{12} \mathrm{O}_{2}$ & Ethyl cinnamate & $115.0548 ; 103.0547 ; 77.039$ \\
\hline $36^{*}$ & 1.95 & $\begin{array}{l}{[\mathrm{M}+} \\
\mathrm{H}]^{+}\end{array}$ & 132.1019 & 132.1019 & -0.025 & $\mathrm{C}_{6} \mathrm{H}_{13} \mathrm{NO}_{2}$ & Leucine or Isoleucine & $132.1016 ; 86.0961$ \\
\hline 37 & 1.96 & $\begin{array}{l}{[\mathrm{M}+} \\
\mathrm{H}]^{+}\end{array}$ & 152.1070 & 152.1070 & -0.041 & $\mathrm{C}_{9} \mathrm{H}_{13} \mathrm{NO}$ & N-Methyltyramine & $103.0549 ; 91.0546 ; 77.0387$ \\
\hline 38 & 2.06 & $\begin{array}{l}{[\mathrm{M}+} \\
\mathrm{H}]^{+}\end{array}$ & 166.1225 & 166.1226 & -0.111 & $\mathrm{C}_{10} \mathrm{H}_{15} \mathrm{NO}$ & Hordenine & $121.0671 ; 103.0556 ; 91.055$ \\
\hline $39 *$ & 2.08 & $\begin{array}{l}{[\mathrm{M}+} \\
\mathrm{H}]^{+}\end{array}$ & 136.0616 & 136.0618 & -0.142 & $\mathrm{C}_{5} \mathrm{H}_{5} \mathrm{~N}_{5}$ & Adenine & $136.0616 ; 119.0355 ; 92.023$ \\
\hline $40 *$ & 2.09 & {$[\mathrm{M}-\mathrm{H}]^{-}$} & 169.0133 & 169.0131 & 0.948 & $\mathrm{C}_{7} \mathrm{H}_{6} \mathrm{O}_{5}$ & Gallic acid & $\begin{array}{l}\text { 169.0127; } 151.0020 ; 125.02 \\
97.0277 ; 81.0327 ; 69.0327\end{array}$ \\
\hline $41^{*}$ & 2.29 & $\begin{array}{l}{[\mathrm{M}+} \\
\mathrm{H}]^{+}\end{array}$ & 137.0457 & 137.0458 & -0.067 & $\mathrm{C}_{5} \mathrm{H}_{4} \mathrm{~N}_{4} \mathrm{O}$ & 6-Hydroxypurine & $\begin{array}{l}137.0442 ; 119.0353 ; 110.03 \\
94.0384\end{array}$ \\
\hline $42^{*}$ & 2.29 & $\begin{array}{l}{[\mathrm{M}+} \\
\mathrm{H}]^{+}\end{array}$ & 152.0565 & 152.0567 & -0.196 & $\mathrm{C}_{5} \mathrm{H}_{5} \mathrm{~N}_{5} \mathrm{O}$ & Guanine & $152.0670 ; 135.0294$ \\
\hline $43^{\star}$ & 2.30 & {$[\mathrm{M}-\mathrm{H}]^{-}$} & 282.0846 & 282.0833 & 4.733 & $\mathrm{C}_{10} \mathrm{H}_{13} \mathrm{~N}_{5} \mathrm{O}_{5}$ & Guanosine & $\begin{array}{l}282.0879 ; 150.0432 ; 133.01 \\
108.0211\end{array}$ \\
\hline $44^{\star}$ & 2.61 & {$[\mathrm{M}-\mathrm{H}]^{-}$} & 266.0896 & 266.0884 & 4.696 & $\mathrm{C}_{10} \mathrm{H}_{13} \mathrm{~N}_{5} \mathrm{O}_{4}$ & Adenosine & $134.0485 ; 107.0382 ; 92.025$ \\
\hline $45^{\star}$ & 2.91 & $\begin{array}{l}{[\mathrm{M}+} \\
\mathrm{H}]^{+}\end{array}$ & 168.1018 & 168.1019 & -0.507 & $\mathrm{C}_{9} \mathrm{H}_{13} \mathrm{NO}_{2}$ & Synephrine & $\begin{array}{l}\text { 168.10; } 150.09 ; 135.07 ; 119 \\
91.05\end{array}$ \\
\hline $46^{\star}$ & 3.26 & {$[\mathrm{M}-\mathrm{H}]^{-}$} & 283.0685 & 283.0673 & 4.237 & $\mathrm{C}_{10} \mathrm{H}_{12} \mathrm{~N}_{4} \mathrm{O}_{6}$ & Xanthosine & $283.0676 ; 151.0267 ; 108.02$ \\
\hline $47^{\star}$ & 3.79 & $\begin{array}{l}{[\mathrm{M}+} \\
\mathrm{H}]^{+}\end{array}$ & 166.0861 & 166.0863 & -0.165 & $\mathrm{C}_{9} \mathrm{H}_{11} \mathrm{NO}_{2}$ & L-Phenylalanine & $\begin{array}{l}\text { 120.0798; } 103.0532 ; 91.053 \\
\text { 79.0539; 77.0377; }\end{array}$ \\
\hline 48 & 3.81 & $\begin{array}{l}{[\mathrm{M}+} \\
\mathrm{H}]^{+}\end{array}$ & 120.0809 & 120.0808 & 0.144 & $\mathrm{C}_{8} \mathrm{H}_{9} \mathrm{~N}$ & N-Benzylidenemethylamine & $120.0804 ; 103.0524 ; 91.053$ \\
\hline 49 & 4.10 & {$[\mathrm{M}-\mathrm{H}]^{-}$} & 305.0669 & 305.0656 & 4.428 & $\mathrm{C}_{15} \mathrm{H}_{14} \mathrm{O}_{7}$ & Leucocyanidin & $305.0627 ; 179.0433 ; 167.03$ \\
\hline 50 & 4.33 & {$[\mathrm{M}-\mathrm{H}]^{-}$} & 153.0182 & 153.0182 & -0.065 & $\mathrm{C}_{7} \mathrm{H}_{6} \mathrm{O}_{4}$ & Protocatechuic acid & $109.0290 ; 108.0220 ; 91.019$ \\
\hline 51 & 4.70 & {$[\mathrm{M}-\mathrm{H}]^{-}$} & 315.0725 & 315.0711 & 4.512 & $\mathrm{C}_{13} \mathrm{H}_{16} \mathrm{O}_{9}$ & Gentisic acid glycoside & $315.0716 ; 152.0116 ; 108.02$ \\
\hline $52^{*}$ & 5.18 & $\begin{array}{l}{[\mathrm{M}+} \\
\mathrm{H}]^{+}\end{array}$ & 152.1069 & 152.1070 & -0.101 & $\mathrm{C}_{9} \mathrm{H}_{13} \mathrm{NO}$ & Phenylpropanolamine & $\begin{array}{l}\text { 152.0574; } 135.0303 ; 121.06 \\
77.0377\end{array}$ \\
\hline $53^{\star}$ & 6.15 & $\begin{array}{l}{[\mathrm{M}+} \\
\mathrm{H}]^{+}\end{array}$ & 152.1069 & 152.1070 & -0.131 & $\mathrm{C}_{9} \mathrm{H}_{13} \mathrm{NO}$ & D-Demethyl pseudoephedrine & $\begin{array}{l}134.0962 ; 115.0555 ; 91.048 \\
65.0371\end{array}$ \\
\hline 54 & 6.16 & {$[\mathrm{M}-\mathrm{H}]^{-}$} & 331.1038 & 331.1024 & 4.233 & $\mathrm{C}_{14} \mathrm{H}_{20} \mathrm{O}_{9}$ & $\begin{array}{l}\text { 2,6-Dimethoxy-4-hydroxyphenol-1-O- } \beta \text {-D- } \\
\text { glucopyranoside }\end{array}$ & $168.0414 ; 153.0187 ; 123.01$ \\
\hline $55^{\star}$ & 6.66 & {$[\mathrm{M}-\mathrm{H}]^{-}$} & 167.0340 & 167.0339 & 0.687 & $\mathrm{C}_{8} \mathrm{H}_{8} \mathrm{O}_{4}$ & Vanillic acid & $152.0116 ; 108.0183$ \\
\hline
\end{tabular}




\begin{tabular}{|c|c|c|c|c|c|c|c|c|}
\hline No. & $\begin{array}{l}\mathrm{RT} \\
\text { /min }\end{array}$ & $\begin{array}{l}\text { lon } \\
\text { model }\end{array}$ & $\begin{array}{l}\text { Measured } \\
\text { mass /Da }\end{array}$ & $\begin{array}{l}\text { Calculated } \\
\text { mass / } \mathrm{Da}\end{array}$ & $\begin{array}{l}\text { Error } \\
\text { /ppm }\end{array}$ & $\begin{array}{l}\text { Molecular } \\
\text { formula }\end{array}$ & Identification & Fragment ions \\
\hline $56^{*}$ & 7.30 & $\begin{array}{l}{[\mathrm{M}+} \\
\mathrm{H}]^{+}\end{array}$ & 166.1225 & 166.1226 & -0.171 & $\mathrm{C}_{10} \mathrm{H}_{15} \mathrm{NO}$ & Ephedrine & $\begin{array}{l}\text { 166.1225; } 148.1119 ; 133.08 \\
117.0701 ; 91.0548\end{array}$ \\
\hline 57 & 7.71 & $\begin{array}{l}{[\mathrm{M}+} \\
\mathrm{FA}-\mathrm{H}]^{-}\end{array}$ & 520.1673 & 520.1661 & 2.344 & $\mathrm{C}_{20} \mathrm{H}_{29} \mathrm{NO}_{12}$ & $\begin{array}{l}\text { L-a-[(6-O-B-D-Glucopyranosyl- } \beta-D- \\
\text { glucopyranosyl)oxy]benzeneacetamide }\end{array}$ & $474.1613 ; 150.0584$ \\
\hline $58^{*}$ & 7.75 & {$[\mathrm{M}-\mathrm{H}]^{-}$} & 203.0819 & 203.0815 & 2.146 & $\mathrm{C}_{11} \mathrm{H}_{12} \mathrm{~N}_{2} \mathrm{O}_{2}$ & Tryptophan & $\begin{array}{l}203.0784 ; 159.0957 ; 142.06 \\
116.0516 ; 74.0254\end{array}$ \\
\hline $59 *$ & 7.77 & {$[\mathrm{M}-\mathrm{H}]^{-}$} & 353.0880 & 353.0867 & 3.601 & $\mathrm{C}_{16} \mathrm{H}_{18} \mathrm{O}_{9}$ & Neochlorogenic acid & $\begin{array}{l}353.0857 ; 191.0563 ; 179.03 \\
135.0454\end{array}$ \\
\hline 60 & 7.99 & {$[\mathrm{M}-\mathrm{H}]-$} & 151.0390 & 151.0390 & 0.029 & $\mathrm{C}_{8} \mathrm{H}_{8} \mathrm{O}_{3}$ & Vanillin & $109.3493 ; 125.0762 ; 65.005$ \\
\hline 61 & 8.05 & {$[\mathrm{M}-\mathrm{H}]-$} & 181.0497 & 181.0495 & 1.131 & $\mathrm{C}_{9} \mathrm{H}_{10} \mathrm{O}_{4}$ & Syringaldehyde & $135.9790 ; 117.0358$ \\
\hline $62^{\star}$ & 8.33 & $\begin{array}{l}{[\mathrm{M}+} \\
\mathrm{H}]^{+}\end{array}$ & 166.1225 & 166.1226 & -0.141 & $\mathrm{C}_{10} \mathrm{H}_{15} \mathrm{NO}$ & Pseudoephedrine & $\begin{array}{l}148.110 ; 133.08777 ; 117.06 \\
91.0534\end{array}$ \\
\hline 63 & 8.49 & $\begin{array}{l}{[\mathrm{M}+} \\
\mathrm{FA}-\mathrm{H}]^{-}\end{array}$ & 520.1672 & 520.1661 & 2.228 & $\mathrm{C}_{20} \mathrm{H}_{29} \mathrm{NO}_{12}$ & $\begin{array}{l}\text { D-a-[(6-O- } \beta \text {-D-Glucopyranosyl- } \beta \text {-D- } \\
\text { glucopyranosyl)oxy]benzeneacetamide }\end{array}$ & $474.1635 ; 313.1103 ; 150.05$ \\
\hline $64^{*}$ & 8.62 & $\begin{array}{l}{[\mathrm{M}+} \\
\mathrm{H}]^{+}\end{array}$ & 180.1381 & 180.1383 & -1.281 & $\mathrm{C}_{11} \mathrm{H}_{17} \mathrm{NO}$ & Methylephedrine & $\begin{array}{l}180.1393 ; 1262.1267 ; 147.1 \\
117.0702 ; 91.0537\end{array}$ \\
\hline 65 & 8.73 & {$[\mathrm{M}-\mathrm{H}]-$} & 285.0618 & 285.0605 & 4.582 & $\mathrm{C}_{12} \mathrm{H}_{14} \mathrm{O}_{8}$ & Uralenneoside & $153.0183 ; 108.0215$ \\
\hline 66 & 8.79 & $\begin{array}{l}{[\mathrm{M}+} \\
\mathrm{H}]^{+}\end{array}$ & 447.1470 & 447.1497 & -6.044 & $\mathrm{C}_{19} \mathrm{H}_{26} \mathrm{O}_{12}$ & Cinnacasolide A & 317.0819; \\
\hline 67 & 8.92 & {$[\mathrm{M}-\mathrm{H}]-$} & 165.0546 & 165.0546 & 0.009 & $\mathrm{C}_{9} \mathrm{H}_{10} \mathrm{O}_{3}$ & Phloretic acid & $119.0506 ; 93.0343$ \\
\hline 68 & 9.22 & {$[\mathrm{M}-\mathrm{H}]^{-}$} & 577.1357 & 577.1341 & 2.924 & $\mathrm{C}_{30} \mathrm{H}_{26} \mathrm{O}_{12}$ & Procyanidin B1 & $\begin{array}{l}425.0916 ; 407.0778 ; 289.07 \\
219.0668 ; 205.0510 ; 161.02 \\
137.0277 ; 125.0255 ;\end{array}$ \\
\hline 69 & 9.39 & {$[\mathrm{M}-\mathrm{H}]^{-}$} & 475.1460 & 475.1446 & 2.910 & $\mathrm{C}_{20} \mathrm{H}_{28} \mathrm{O}_{13}$ & L-Amygdalinic acid & $\begin{array}{l}475.1452 ; 431.1560 ; 269.10 \\
161.0453 ; 101.0250\end{array}$ \\
\hline 70 & 10.43 & $\begin{array}{l}{[\mathrm{M}+} \\
\mathrm{H}]^{+}\end{array}$ & 272.1278 & 272.1281 & -1.359 & $\mathrm{C}_{16} \mathrm{H}_{17} \mathrm{NO}_{3}$ & Higenamine & $\begin{array}{l}107.0512 ; 115.0564 ; 143.05 \\
161.0626\end{array}$ \\
\hline 71 & 10.53 & {$[\mathrm{M}-\mathrm{H}]^{-}$} & 121.0282 & 121.0284 & -0.246 & $\mathrm{C}_{7} \mathrm{H}_{6} \mathrm{O}_{2}$ & 4-Hydroxybenzaldehyde & $121.0305 ; 92.0251$ \\
\hline 72 & 10.65 & {$[\mathrm{M}-\mathrm{H}]^{-}$} & 577.1358 & 577.1341 & 3.028 & $\mathrm{C}_{30} \mathrm{H}_{26} \mathrm{O}_{12}$ & Procyanidin B3 & $\begin{array}{l}451.1160 ; 407.0712 ; 289.07 \\
125.0252 ;\end{array}$ \\
\hline $73^{*}$ & 10.86 & {$[\mathrm{M}-\mathrm{H}]^{-}$} & 289.0719 & 289.0707 & 4.377 & $\mathrm{C}_{15} \mathrm{H}_{14} \mathrm{O}_{6}$ & Catechin & $\begin{array}{l}289.0702 ; 245.0818 ; 123.04 \\
109.0297\end{array}$ \\
\hline 74 & 10.99 & $\begin{array}{l}{[\mathrm{M}+} \\
\mathrm{H}]^{+}\end{array}$ & 217.0970 & 217.0972 & -0.572 & $\mathrm{C}_{12} \mathrm{H}_{12} \mathrm{~N}_{2} \mathrm{O}_{2}$ & Anoectochine & $\begin{array}{l}217.0973 ; 169.0758 ; 141.11 \\
144.0827 ; 127.0560 ;\end{array}$ \\
\hline 75 & 11.08 & {$[\mathrm{M}-\mathrm{H}]^{-}$} & 463.1462 & 463.1446 & 3.439 & $\mathrm{C}_{19} \mathrm{H}_{28} \mathrm{O}_{13}$ & Cinnacassoside C & $\begin{array}{l}293.1080 ; 233.0642 ; 149.04 \\
\text { 139.0032; }\end{array}$ \\
\hline 76 & 11.60 & {$[\mathrm{M}-\mathrm{H}]^{-}$} & 303.0512 & 303.0499 & 4.128 & $\mathrm{C}_{15} \mathrm{H}_{12} \mathrm{O}_{7}$ & $\begin{array}{l}\text { (2R,3R)-3,5,7,2 ',6' - } \\
\text { Pentahydroxyflavanone }\end{array}$ & $\begin{array}{l}303.0511 ; 217.0537 ; 177.02 \\
149.0245 ; 125.0250\end{array}$ \\
\hline 77 & 11.9 & {$[\mathrm{M}-\mathrm{H}]^{-}$} & 299.0775 & 299.0761 & 4.468 & $\mathrm{C}_{13} \mathrm{H}_{16} \mathrm{O}_{8}$ & Salicylic acid 2-O- $\beta$-D-glucoside & $299.0766 ; 137.0248 ; 93.034$ \\
\hline 78 & 11.95 & {$[\mathrm{M}-\mathrm{H}]^{-}$} & 475.1459 & 475.1446 & 2.700 & $\mathrm{C}_{20} \mathrm{H}_{28} \mathrm{O}_{13}$ & D-Amygdalinic acid & $\begin{array}{l}475.1545 ; 431.1581 ; 151.03 \\
101.0259\end{array}$ \\
\hline $79 *$ & 12.03 & {$[\mathrm{M}-\mathrm{H}]^{-}$} & 179.0340 & 179.0339 & 0.865 & $\mathrm{C}_{9} \mathrm{H}_{8} \mathrm{O}_{4}$ & Caffeic acid & 135.045 \\
\hline 80 & 12.06 & $\begin{array}{l}{[\mathrm{M}+} \\
\mathrm{H}]^{+}\end{array}$ & 137.1072 & 137.1073 & -0.145 & $\mathrm{C}_{8} \mathrm{H}_{12} \mathrm{~N}_{2}$ & Ligustrazine & $\begin{array}{l}137.1072 ; 122.0850 ; 96.080 \\
80.0483 ; 69.0692 ; 55.0549\end{array}$ \\
\hline $81^{*}$ & 12.51 & {$[\mathrm{M}-\mathrm{H}]^{-}$} & 353.0880 & 353.0867 & 3.516 & $\mathrm{C}_{16} \mathrm{H}_{18} \mathrm{O}_{9}$ & Chlorogenic acid & $\begin{array}{l}353.0883 ; 191.0549 ; 179.03 \\
173.0442 ; 161.0228 ; 155.03 \\
135.0435\end{array}$ \\
\hline 82 & 12.59 & $\begin{array}{l}{[\mathrm{M}+} \\
\mathrm{FA}-\mathrm{H}]^{-}\end{array}$ & 535.1671 & 535.1657 & 2.436 & $\mathrm{C}_{21} \mathrm{H}_{30} \mathrm{O}_{13}$ & Tectoruside & 165.0578 \\
\hline 83 & 12.98 & $\begin{array}{l}{[\mathrm{M}+} \\
\text { FA-H] }\end{array}$ & 373.1142 & 373.1129 & 3.502 & $\mathrm{C}_{15} \mathrm{H}_{20} \mathrm{O}_{8}$ & Androsin & $165.0574 ; 150.0343$ \\
\hline
\end{tabular}




\begin{tabular}{|c|c|c|c|c|c|c|c|c|}
\hline No. & $\begin{array}{l}\mathrm{RT} \\
/ \mathrm{min}\end{array}$ & $\begin{array}{l}\text { lon } \\
\text { model }\end{array}$ & $\begin{array}{l}\text { Measured } \\
\text { mass /Da }\end{array}$ & $\begin{array}{l}\text { Calculated } \\
\text { mass /Da }\end{array}$ & $\begin{array}{l}\text { Error } \\
\text { /ppm }\end{array}$ & $\begin{array}{l}\text { Molecular } \\
\text { formula }\end{array}$ & Identification & Fragment ions \\
\hline 84 & 13.18 & {$[\mathrm{M}-\mathrm{H}]^{-}$} & 367.1035 & 367.1024 & 3.082 & $\mathrm{C}_{17} \mathrm{H}_{20} \mathrm{O}_{9}$ & 3-Feruloylquinic acid & $\begin{array}{l}\text { 134.0387; } 193.0530 ; 117.03 \\
\text { 191.0588 }\end{array}$ \\
\hline 85 & 13.51 & {$[\mathrm{M}-\mathrm{H}]^{-}$} & 577.1355 & 577.1341 & 2.508 & $\mathrm{C}_{30} \mathrm{H}_{26} \mathrm{O}_{12}$ & Procyanidin B2 & $\begin{array}{l}451.1007 ; 425.0845 ; 407.08 \\
289.0729 ; 161.0264 ; 125.02\end{array}$ \\
\hline 86 & 13.62 & $\begin{array}{l}{[\mathrm{M}+} \\
\text { FA-H] }\end{array}$ & 293.1244 & 293.1231 & 4.421 & $\mathrm{C}_{11} \mathrm{H}_{20} \mathrm{O}_{6}$ & Pent-4-enyl-D-glucopyranoside & 293.1216; \\
\hline $87 *$ & 13.74 & {$[\mathrm{M}-\mathrm{H}]^{-}$} & 353.0880 & 353.0867 & 3.516 & $\mathrm{C}_{16} \mathrm{H}_{18} \mathrm{O}_{9}$ & Cryptochlorogenic acid & $\begin{array}{l}353.0891 ; 191.0572 ; 179.03 \\
173.0470 ; 135.0459\end{array}$ \\
\hline 88 & 13.80 & {$[\mathrm{M}-\mathrm{H}]-$} & 443.1924 & 443.1912 & 2.655 & $\mathrm{C}_{21} \mathrm{H}_{32} \mathrm{O}_{10}$ & $\begin{array}{l}\text { Dihydrophaseic acid 4'-O- } \beta- \\
\text { glucopyranoside }\end{array}$ & $\begin{array}{l}443.1951 ; 119.0356 ; 89.024 \\
59.0144\end{array}$ \\
\hline 89 & 13.82 & $\begin{array}{l}{[\mathrm{M}+} \\
\mathrm{FA}-\mathrm{H}]^{-}\end{array}$ & 493.2294 & 493.2280 & 2.873 & $\mathrm{C}_{21} \mathrm{H}_{36} \mathrm{O}_{10}$ & Atractyloside A & $\begin{array}{l}447.2232 ; 285.1700 ; 179.05 \\
131.0359 ; 119.0362 ;\end{array}$ \\
\hline $90 *$ & 13.93 & $\begin{array}{l}{[\mathrm{M}+} \\
\mathrm{FA}-\mathrm{H}]^{-}\end{array}$ & 502.1568 & 502.1555 & 2.616 & $\mathrm{C}_{20} \mathrm{H}_{27} \mathrm{NO}_{11}$ & Amygdalin & $\begin{array}{l}502.1567 ; 323.0972 ; 263.07 \\
221.0661 ; 179.0550 ; ; 161.0 \\
119.0335 ; 101.0229 ; 89.022\end{array}$ \\
\hline 91 & 14.07 & $\begin{array}{l}{[\mathrm{M}+} \\
\mathrm{FA}-\mathrm{H}]^{-}\end{array}$ & 477.1616 & 477.1603 & 2.709 & $\mathrm{C}_{19} \mathrm{H}_{28} \mathrm{O}_{11}$ & Benzyl- $\beta$-gentiobioside & 431.157269 .103161 .04510 \\
\hline 92 & 14.16 & {$[\mathrm{M}-\mathrm{H}]^{-}$} & 431.1559 & 431.1548 & 2.463 & $\mathrm{C}_{19} \mathrm{H}_{28} \mathrm{O}_{11}$ & Osmanthuside $\mathrm{H}$ or isomer & $431.1150 ; 125.0234$ \\
\hline $93^{*}$ & 14.22 & {$[\mathrm{M}-\mathrm{H}]^{-}$} & 294.0985 & 294.0972 & 4.510 & $\mathrm{C}_{14} \mathrm{H}_{17} \mathrm{NO}_{6}$ & Prunasin & $\begin{array}{l}\text { 161.0435; } 101.0242 ; 85.030 \\
71.0141\end{array}$ \\
\hline 94 & 14.38 & $\begin{array}{l}{[\mathrm{M}+} \\
\mathrm{H}]^{+}\end{array}$ & 193.0857 & 193.0859 & -0.936 & $\mathrm{C}_{11} \mathrm{H}_{12} \mathrm{O}_{3}$ & Myristicin & $133.0628 ; 147.0830$ \\
\hline 95 & 14.73 & $\begin{array}{l}{[\mathrm{M}+} \\
\mathrm{H}]^{+}\end{array}$ & 179.0337 & 179.0339 & -0.923 & $\mathrm{C} 9 \mathrm{H} 6 \mathrm{O} 4$ & 4,7-Dihydroxycoumarin & $\begin{array}{l}\text { 179.03354; } 163.03850 ; \\
145.02814\end{array}$ \\
\hline $96 *$ & 15.04 & {$[\mathrm{M}-\mathrm{H}]^{-}$} & 289.0720 & 289.0707 & 4.481 & $\mathrm{C}_{15} \mathrm{H}_{14} \mathrm{O}_{6}$ & Epicatechin & $\begin{array}{l}289.0723 ; 245.0813 ; 188.04 \\
123.0453\end{array}$ \\
\hline 97 & 15.22 & $\begin{array}{l}{[\mathrm{M}+} \\
\mathrm{H}]^{+}\end{array}$ & 179.0336 & 179.0339 & -1.481 & $\mathrm{C}_{9} \mathrm{H}_{6} \mathrm{O}_{4}$ & 6,7-Dihydroxycoumarin & $\begin{array}{l}\text { 179.03357; } 163.03873 ; \\
145.02832\end{array}$ \\
\hline $98^{*}$ & 15.29 & {$[\mathrm{M}-\mathrm{H}]^{-}$} & 515.1197 & 515.1184 & 2.499 & $\mathrm{C}_{25} \mathrm{H}_{24} \mathrm{O}_{12}$ & 1,3-O-Dicaffeoylquinic acid & $\begin{array}{l}515.1199 ; 353.0858 ; 335.07 \\
191.0562\end{array}$ \\
\hline 99 & 15.36 & $\begin{array}{l}{[\mathrm{M}+} \\
\mathrm{H}]^{+}\end{array}$ & 303.0495 & 303.0499 & -1.317 & $\mathrm{C}_{15} \mathrm{H}_{10} \mathrm{O}_{7}$ & $\begin{array}{l}\text { Herbacetin or } 3,5,7,2,6 \text { - } \\
\text { pentahydroxyflavone }\end{array}$ & $\begin{array}{l}303.0483 ; 257.0466 ; 229.04 \\
153.0187 ; 149.0241\end{array}$ \\
\hline 100 & 15.55 & $\begin{array}{l}{[\mathrm{M}+} \\
\mathrm{H}]^{+}\end{array}$ & 251.1024 & 251.1026 & -1.089 & $\mathrm{C}_{12} \mathrm{H}_{14} \mathrm{~N}_{2} \mathrm{O}_{4}$ & Crotonine $\mathrm{A}$ & $251.1026 ; 233.0930 ; 215.08$ \\
\hline 101 & 15.58 & {$[\mathrm{M}-\mathrm{H}]-$} & 163.0391 & 163.0390 & 0.139 & $\mathrm{C}_{9} \mathrm{H}_{8} \mathrm{O}_{3}$ & p-Coumaric acid & $119.0518 ; 93.0347$ \\
\hline 102 & 15.79 & $\begin{array}{l}{[\mathrm{M}+} \\
\mathrm{FA}-\mathrm{H}]^{-}\end{array}$ & 502.1570 & 502.1555 & 2.975 & $\mathrm{C}_{20} \mathrm{H}_{27} \mathrm{NO}_{11}$ & Amygdalin isomer & $\begin{array}{l}456.1532 ; 323.0988 ; 221.06 \\
161.0460 ; 119.0357 ; 89.024\end{array}$ \\
\hline 103 & 15.89 & {$[\mathrm{M}-\mathrm{H}]-$} & 295.1189 & 295.1176 & 4.355 & $\mathrm{C}_{15} \mathrm{H}_{20} \mathrm{O}_{6}$ & Rosin & $\begin{array}{l}\text { 161.0453; } 133.0663 ; 113.02 \\
101.0239\end{array}$ \\
\hline 104 & 15.92 & {$[\mathrm{M}-\mathrm{H}]^{-}$} & 161.0233 & 161.0233 & 0.009 & $\mathrm{C}_{9} \mathrm{H}_{6} \mathrm{O}_{3}$ & Umbelliferone & $\begin{array}{l}\text { 133.0308; } 105.0341 ; 77.039 \\
89.0406 ; 65.0037\end{array}$ \\
\hline 105 & 16 & {$[\mathrm{M}-\mathrm{H}]^{-}$} & 207.0293 & 207.0288 & 2.561 & $\mathrm{C}_{10} \mathrm{H}_{8} \mathrm{O}_{5}$ & Fraxetin & $207.0323 ; 164.5855 ; 108.02$ \\
\hline 106 & 16.02 & $\begin{array}{l}{[\mathrm{M}+} \\
\mathrm{FA}-\mathrm{H}]^{-}\end{array}$ & 711.2151 & 711.2190 & -5.447 & $\mathrm{C}_{24} \mathrm{H}_{42} \mathrm{O}_{21}$ & Stachyose & $\begin{array}{l}665.2138 ; 485.1515 ; 341.10 \\
179.0557\end{array}$ \\
\hline 107 & 16.2 & {$[\mathrm{M}-\mathrm{H}]^{-}$} & 421.0779 & 421.0765 & 3.330 & $\mathrm{C}_{19} \mathrm{H}_{18} \mathrm{O}_{11}$ & Mangiferin & $331.0461 ; 301.0361 ; 272.03$ \\
\hline 108 & 16.29 & {$[\mathrm{M}-\mathrm{H}]^{-}$} & 327.1090 & 327.1074 & 3.932 & $\mathrm{C}_{15} \mathrm{H}_{20} \mathrm{O}_{8}$ & Dihydrocinnacasside & $\begin{array}{l}\text { 165.0569; } 147.0449 ; 121.06 \\
\text { 106.0451; }\end{array}$ \\
\hline 109 & 16.34 & $\begin{array}{l}{[\mathrm{M}+} \\
\mathrm{H}]^{+}\end{array}$ & 625.1759 & 625.1763 & -0.659 & $\mathrm{C}_{28} \mathrm{H}_{32} \mathrm{O}_{16}$ & Stellarin-2 & $\begin{array}{l}625.1707 ; 607.1640 ; 589.15 \\
487.1207\end{array}$ \\
\hline 110 & 16.38 & $\begin{array}{l}{[\mathrm{M}+} \\
\mathrm{H}]^{+}\end{array}$ & 336.1803 & 336.1805 & -0.653 & $\mathrm{C}_{18} \mathrm{H}_{25} \mathrm{NO}_{5}$ & Senecionine & $\begin{array}{l}308.1788 ; 138.0912 ; 120.08 \\
94.0642\end{array}$ \\
\hline
\end{tabular}




\begin{tabular}{|c|c|c|c|c|c|c|c|c|}
\hline No. & $\begin{array}{l}\mathrm{RT} \\
/ \mathrm{min}\end{array}$ & $\begin{array}{l}\text { lon } \\
\text { model }\end{array}$ & $\begin{array}{l}\text { Measured } \\
\text { mass /Da }\end{array}$ & $\begin{array}{l}\text { Calculated } \\
\text { mass /Da }\end{array}$ & $\begin{array}{l}\text { Error } \\
\text { /ppm }\end{array}$ & $\begin{array}{l}\text { Molecular } \\
\text { formula }\end{array}$ & Identification & Fragment ions \\
\hline 111 & 16.49 & {$[\mathrm{M}-\mathrm{H}]^{-}$} & 367.1036 & 367.1024 & 3.491 & $\mathrm{C}_{17} \mathrm{H}_{20} \mathrm{O}_{9}$ & 4-Feruloylquinic acid & $\begin{array}{l}\text { 193.0522; } 191.0578 ; 173.04 \\
134.0387 ; 93.0348\end{array}$ \\
\hline 112 & 16.65 & $\begin{array}{l}{[\mathrm{M}+} \\
\mathrm{H}]^{+}\end{array}$ & 566.2010 & 566.2012 & -0.401 & $\mathrm{C}_{25} \mathrm{H}_{32} \mathrm{~N}_{5} \mathrm{O}_{8} \mathrm{Cl}$ & Astin L & $566.2131 ; 423.1469$ \\
\hline 113 & 16.75 & {$[\mathrm{M}-\mathrm{H}]^{-}$} & 367.1036 & 367.1024 & 3.491 & $\mathrm{C}_{17} \mathrm{H}_{20} \mathrm{O}_{9}$ & 5-Feruloylquinic acid & $\begin{array}{l}\text { 191.0583; } 173.0485 ; 134.03 \\
\text { 93.0346; }\end{array}$ \\
\hline 114 & 16.75 & {$[\mathrm{M}-\mathrm{H}]^{-}$} & 577.1357 & 577.1341 & 2.924 & $\mathrm{C}_{30} \mathrm{H}_{26} \mathrm{O}_{12}$ & Procyanidin B4 & $\begin{array}{l}559.1291 ; 451.1081 ; 425.08 \\
407.0738 ; 289.0696 ; 273.04 \\
201.0526 ; 125.0234 ;\end{array}$ \\
\hline 115 & 16.82 & $\begin{array}{l}{[\mathrm{M}+} \\
\mathrm{H}]^{+}\end{array}$ & 193.0494 & 193.0495 & -0.753 & $\mathrm{C}_{10} \mathrm{H}_{8} \mathrm{O}_{4}$ & Isoscopoletin & $\begin{array}{l}178.0294 ; 150.0337 ; 133.03 \\
122.0383\end{array}$ \\
\hline 116 & 17.03 & {$[\mathrm{M}-\mathrm{H}]^{-}$} & 593.1517 & 593.1501 & 2.653 & $\mathrm{C}_{27} \mathrm{H}_{30} \mathrm{O}_{15}$ & Vicenin-li & $\begin{array}{l}\text { 593.1520; 503.1167; } 473.10 \\
383.0748 ; 353.0645\end{array}$ \\
\hline $117 *$ & 17.36 & $\begin{array}{l}{[\mathrm{M}+} \\
\mathrm{H}]^{+}\end{array}$ & 195.0649 & 195.0652 & -1.258 & $\mathrm{C}_{10} \mathrm{H}_{10} \mathrm{O}_{4}$ & Ferulic acid & $\begin{array}{l}177.0534 ; 145.0281 ; 117.03 \\
89.0380\end{array}$ \\
\hline 118 & 17.36 & $\begin{array}{l}{[\mathrm{M}+} \\
\mathrm{H}]^{+}\end{array}$ & 179.0336 & 179.0339 & -1.593 & $\mathrm{C}_{9} \mathrm{H}_{6} \mathrm{O}_{4}$ & 5,7-Dihydroxycoumarin & $\begin{array}{l}\text { 179.03358; } 163.03880 \\
145.02818\end{array}$ \\
\hline 119 & 17.5 & $\begin{array}{l}{[\mathrm{M}+} \\
\mathrm{H}]^{+}\end{array}$ & 251.1387 & 251.1642 & -1.398 & $\mathrm{C}_{15} \mathrm{H}_{22} \mathrm{O}_{3}$ & $1 \beta, 11$-Dihydroxy- $\beta$-cyperone & $\begin{array}{l}\text { 72.0801; } 84.9591 ; 110.0605 \\
\text { 140.9194; } 198.1117 ; 219.04 \\
\text { 180.9492; }\end{array}$ \\
\hline 120 & 17.60 & $\begin{array}{l}{[\mathrm{M}+} \\
\mathrm{H}]^{+}\end{array}$ & 625.1758 & 625.1763 & -0.850 & $\mathrm{C}_{28} \mathrm{H}_{32} \mathrm{O}_{16}$ & 4'-Methoxy-lucenin-2 & $\begin{array}{l}625.1632 ; 607.1759 ; 589.15 \\
487.1216\end{array}$ \\
\hline 121 & 17.66 & {$[\mathrm{M}-\mathrm{H}]^{-}$} & 459.1149 & 459.1133 & 3.382 & $\mathrm{C}_{19} \mathrm{H}_{24} \mathrm{O}_{13}$ & Parishin E & $\begin{array}{l}307.0650 ; 208.0377 ; 164.05 \\
151.0398 ; 101.0250\end{array}$ \\
\hline 122 & 17.69 & $\begin{array}{l}{[\mathrm{M}+} \\
\mathrm{H}]+\end{array}$ & 223.0600 & 223.0601 & -0.672 & $\mathrm{C}_{11} \mathrm{H}_{10} \mathrm{O}_{5}$ & Saikochromone A or its isomers & $\begin{array}{l}223.0623 ; 207.0304 ; 190.02 \\
179.0358 ; 162.0332 ; 134.03 \\
105.0341 ; 78.0469\end{array}$ \\
\hline 123 & 17.73 & $\begin{array}{l}{[\mathrm{M}+} \\
\mathrm{H}]^{+}\end{array}$ & 403.1360 & 403.1387 & -6.832 & $\mathrm{C}_{21} \mathrm{H}_{22} \mathrm{O}_{8}$ & Hexamethoxyfalvone & $\begin{array}{l}403.1390 ; 388.1170 ; 373.01 \\
327.0863\end{array}$ \\
\hline 124 & 17.85 & $\begin{array}{l}{[\mathrm{M}+} \\
\mathrm{H}]^{+}\end{array}$ & 366.1909 & 366.1911 & -0.612 & $\mathrm{C}_{19} \mathrm{H}_{27} \mathrm{NO}_{6}$ & Senkirkine or its isomer & $\begin{array}{l}366.1893 ; 168.1009 ; 150.09 \\
122.0960\end{array}$ \\
\hline 125 & 17.86 & {$[\mathrm{M}-\mathrm{H}]^{-}$} & 563.1410 & 563.1395 & 2.589 & $\mathrm{C}_{26} \mathrm{H}_{28} \mathrm{O}_{14}$ & 8/6-CHexosyl-6/8-C-pentasy 1 apigenin & $\begin{array}{l}563.1415 ; 473.1092 ; 383.07 \\
353.0667 ; 297.0743\end{array}$ \\
\hline 126 & 17.99 & {$[\mathrm{M}-\mathrm{H}]^{-}$} & 435.0934 & 435.0922 & 2.878 & $\mathrm{C}_{20} \mathrm{H}_{20} \mathrm{O}_{11}$ & 7-O-Methylmangiferin & $315.0524 ; 287.0221 ; 272.03$ \\
\hline $127^{*}$ & 18.01 & {$[\mathrm{M}-\mathrm{H}]^{-}$} & 417.1195 & 417.1180 & 3.527 & $\mathrm{C}_{21} \mathrm{H}_{22} \mathrm{O}_{9}$ & Neoliquiritin & $\begin{array}{l}417.1194 ; 255.0803 ; 135.00 \\
119.0497\end{array}$ \\
\hline 128 & 18.15 & {$[\mathrm{M}-\mathrm{H}]^{-}$} & 581.2243 & 581.2229 & 2.465 & $\mathrm{C}_{28} \mathrm{H}_{38} \mathrm{O}_{13}$ & (+)-Lyoniresinol 9'-O-glucoside & $\begin{array}{l}\text { 419.1663; 404.1436; } 343.13 \\
\text { 233.0878; 202.0693; } 153.05 \\
\text { 119.0358; }\end{array}$ \\
\hline $129 *$ & 18.23 & {$[\mathrm{M}-\mathrm{H}]^{-}$} & 137.0232 & 137.0233 & -0.111 & $\mathrm{C}_{7} \mathrm{H}_{6} \mathrm{O}_{3}$ & 3,4-Dihydroxybenzaldehyde & 137.0242; \\
\hline 130 & 18.24 & {$[\mathrm{M}-\mathrm{H}]^{-}$} & 295.0459 & 295.0448 & 3.580 & $\mathrm{C}_{13} \mathrm{H}_{12} \mathrm{O}_{8}$ & Coumaroyltartaric acid & $173.0106 ; 154.9996 ; 111.00$ \\
\hline $131 *$ & 18.30 & {$[\mathrm{M}-\mathrm{H}]^{-}$} & 447.0935 & 447.0922 & 3.024 & $\mathrm{C}_{21} \mathrm{H}_{20} \mathrm{O}_{11}$ & Luteolin-7-O- $\beta$-D-glucoside & $\begin{array}{l}447.0961 ; 357.0596 ; 327.04 \\
285.0434 ; 133.0314\end{array}$ \\
\hline 132 & 18.40 & {$[\mathrm{M}-\mathrm{H}]^{-}$} & 563.1409 & 563.1395 & 2.376 & $\mathrm{C}_{26} \mathrm{H}_{28} \mathrm{O}_{14}$ & Schaftoside & $\begin{array}{l}563.1418 ; 503.1114 ; 383.07 \\
353.0676\end{array}$ \\
\hline 133 & 18.51 & {$[\mathrm{M}-\mathrm{H}]^{-}$} & 415.1613 & 415.1599 & 3.316 & $\mathrm{C}_{19} \mathrm{H}_{28} \mathrm{O}_{10}$ & Icariside Dc & $\begin{array}{l}\text { 397.2059; 233.0752; } 191.05 \\
\text { 161.0462; 149.0445; } 119.03 \\
99.0075 ; 89.0250 ;\end{array}$ \\
\hline 134 & 18.51 & {$[\mathrm{M}-\mathrm{H}]^{-}$} & 549.1617 & 549.1603 & 2.682 & $\mathrm{C}_{26} \mathrm{H}_{30} \mathrm{O}_{13}$ & $\begin{array}{l}\text { Naringenin 7-O-(2- } \beta-D-A p i o f u r a n o s y l)-\beta-D- \\
\text { glucopyranoside }\end{array}$ & $\begin{array}{l}549.1621 ; 429.1106 ; 255.06 \\
135.0100\end{array}$ \\
\hline $135^{\star}$ & 18.59 & {$[\mathrm{M}-\mathrm{H}]^{-}$} & 417.1195 & 417.1180 & 3.527 & $\mathrm{C}_{21} \mathrm{H}_{22} \mathrm{O}_{9}$ & Liquiritin & $\begin{array}{l}417.1195 ; 255.0654 ; 153.01 \\
135.0071 ; 119.0484\end{array}$ \\
\hline 136 & 18.71 & $\begin{array}{l}{[\mathrm{M}+} \\
\mathrm{H}]^{+}\end{array}$ & 366.1907 & 366.1911 & -1.131 & $\mathrm{C}_{19} \mathrm{H}_{27} \mathrm{NO}_{6}$ & Senkirkine or its isomer & $\begin{array}{l}366.1892 ; 168.1018 ; 150.09 \\
122.0595\end{array}$ \\
\hline
\end{tabular}




\begin{tabular}{|c|c|c|c|c|c|c|c|c|}
\hline No. & $\begin{array}{l}\mathrm{RT} \\
/ \mathrm{min}\end{array}$ & $\begin{array}{l}\text { lon } \\
\text { model }\end{array}$ & $\begin{array}{l}\text { Measured } \\
\text { mass /Da }\end{array}$ & $\begin{array}{l}\text { Calculated } \\
\text { mass } / \mathrm{Da}\end{array}$ & $\begin{array}{l}\text { Error } \\
\text { /ppm }\end{array}$ & $\begin{array}{l}\text { Molecular } \\
\text { formula }\end{array}$ & Identification & Fragment ions \\
\hline 137 & 18.82 & {$[\mathrm{M}-\mathrm{H}]^{-}$} & 595.1673 & 595.1657 & 2.610 & $\mathrm{C}_{27} \mathrm{H}_{32} \mathrm{O}_{15}$ & Neoeriocitrin & $\begin{array}{l}595.1671 ; 459.1117 ; 287.05 \\
151.0031 ; 135.0442\end{array}$ \\
\hline 138 & 18.85 & $\begin{array}{l}{[\mathrm{M}+} \\
\mathrm{H}]^{+}\end{array}$ & 550.2059 & 550.2063 & -0.786 & $\mathrm{C}_{25} \mathrm{H}_{32} \mathrm{~N}_{5} \mathrm{O}_{7} \mathrm{Cl}$ & Astin $\mathrm{H}$ or astin $\mathrm{E}$ & $550.2035 ; 131.0473$ \\
\hline 139 & 18.86 & {$[\mathrm{M}-\mathrm{H}]^{-}$} & 741.2260 & 741.2237 & 3.096 & $\mathrm{C}_{33} \mathrm{H}_{42} \mathrm{O}_{19}$ & Naringenin-7-O-triglycoside & $\begin{array}{l}\text { 741.2304; 621.1685; } 339.07 \\
271.0611 ; 151.0034\end{array}$ \\
\hline 140 & 18.94 & $\begin{array}{l}{[2 \mathrm{M}-} \\
\mathrm{H}]-\end{array}$ & 279.0146 & 279.0135 & 3.750 & $\mathrm{C}_{6} \mathrm{H}_{4} \mathrm{O}_{4}$ & Coumaric acid & $132.0226 ; 104.0295 ; 95.950$ \\
\hline 141 & 18.96 & {$[\mathrm{M}-\mathrm{H}]^{-}$} & 549.1617 & 549.1603 & 2.573 & $\mathrm{C}_{26} \mathrm{H}_{30} \mathrm{O}_{13}$ & Liquiritin apiroside & $549.1631 ; 255.0669 ; 135.00$ \\
\hline $142^{\star}$ & 18.98 & {$[\mathrm{M}-\mathrm{H}]^{-}$} & 431.0985 & 431.0973 & 2.799 & $\mathrm{C}_{21} \mathrm{H}_{20} \mathrm{O}_{10}$ & Vitexin & $\begin{array}{l}\text { 431.0943; } 283.0475 ; 249.06 \\
181.0308 ; 102.9854\end{array}$ \\
\hline $143^{*}$ & 19.24 & {$[\mathrm{M}-\mathrm{H}]^{-}$} & 515.1196 & 515.1184 & 2.383 & $\mathrm{C}_{25} \mathrm{H}_{24} \mathrm{O}_{12}$ & Isochlorogenic acid B & $\begin{array}{l}515.1236 ; 353.0895 ; 191.05 \\
179.0356 ; 173.0463\end{array}$ \\
\hline 144 & 19.28 & {$[\mathrm{M}-\mathrm{H}]^{-}$} & 595.1673 & 595.1657 & 2.610 & $\mathrm{C}_{27} \mathrm{H}_{32} \mathrm{O}_{15}$ & Eriocitrin & $595.1664 ; 459.1188 ; 287.05$ \\
\hline 145 & 19.34 & {$[\mathrm{M}-\mathrm{H}]^{-}$} & 547.1461 & 547.1446 & 2.637 & $\mathrm{C}_{26} \mathrm{H}_{28} \mathrm{O}_{13}$ & Chrysin 6-CPen-8-CHex & $\begin{array}{l}\text { 547.1464; 457.1224; } 427.10 \\
\text { 367.0862; 337.0725; }\end{array}$ \\
\hline 146 & 19.36 & {$[\mathrm{M}-\mathrm{H}]^{-}$} & 463.1252 & 463.1235 & 3.654 & $\mathrm{C}_{22} \mathrm{H}_{24} \mathrm{O}_{11}$ & Hesperetin-O-glucoside & $\begin{array}{l}\text { 463.1165; 337.0927; } 160.01 \\
125.0244 ; 83.0141\end{array}$ \\
\hline 147 & 19.44 & {$[\mathrm{M}-\mathrm{H}]^{-}$} & 563.1409 & 563.1395 & 2.483 & $\mathrm{C}_{26} \mathrm{H}_{28} \mathrm{O}_{14}$ & Isoschaftoside & $\begin{array}{l}563.1441 ; 44.1003 ; 353.068 \\
297.0770\end{array}$ \\
\hline 148 & 19.51 & {$[\mathrm{M}-\mathrm{H}]^{-}$} & 547.1459 & 547.1446 & 2.418 & $\mathrm{C}_{26} \mathrm{H}_{28} \mathrm{O}_{13}$ & $\begin{array}{l}\text { Chrysin- } 6-C-a-L-A r a b i n o s i d e-8-C-\beta-D- \\
\text { glucoside }\end{array}$ & $\begin{array}{l}\text { 547.1482; } 457.1150 ; 427.09 \\
\text { 367.0823; 337.0723; }\end{array}$ \\
\hline 149 & 19.52 & $\begin{array}{l}{[\mathrm{M}+} \\
\mathrm{H}]^{+}\end{array}$ & 502.2657 & 502.2660 & -0.697 & $\mathrm{C}_{25} \mathrm{H}_{35} \mathrm{~N}_{5} \mathrm{O}_{6}$ & Astin $\mathrm{G}$ & $502.2646 ; 270.1459 ; 155.12$ \\
\hline 150 & 19.6 & $\begin{array}{l}{[\mathrm{M}+} \\
\mathrm{H}]^{+}\end{array}$ & 301.0700 & 301.0707 & -2.075 & $\mathrm{C}_{16} \mathrm{H}_{12} \mathrm{O}_{6}$ & Pratensein & $\begin{array}{l}285.0422 ; 151.0406 ; 134.03 \\
153.0194\end{array}$ \\
\hline 151 & 19.6 & $\begin{array}{l}{[\mathrm{M}+} \\
\mathrm{H}]^{+}\end{array}$ & 301.0704 & 301.0707 & -2.075 & $\mathrm{C}_{16} \mathrm{H}_{12} \mathrm{O}_{6}$ & Rhamnocitrin & $231.0673 ; 167.0357$ \\
\hline 152 & 19.60 & $\begin{array}{l}{[\mathrm{M}+} \\
\mathrm{H}]^{+}\end{array}$ & 463.1228 & 463.1235 & -1.485 & $\mathrm{C}_{22} \mathrm{H}_{22} \mathrm{O}_{11}$ & Diosmetin-7-O-glucoside & $463.0650 ; 301.0707 ; 286.04$ \\
\hline $153^{\star}$ & 19.61 & {$[\mathrm{M}-\mathrm{H}]^{-}$} & 461.1093 & 461.1078 & 3.128 & $\mathrm{C}_{22} \mathrm{H}_{22} \mathrm{O}_{11}$ & Tectoridin & $\begin{array}{l}461.1084 ; 299.0548 ; 283.02 \\
255.0275\end{array}$ \\
\hline 154 & 19.67 & {$[\mathrm{M}-\mathrm{H}]^{-}$} & 147.0440 & 147.0441 & -0.046 & $\mathrm{C}_{9} \mathrm{H}_{8} \mathrm{O}_{2}$ & 2-Hydroxycinnamaldehyde & 119.0516 \\
\hline $155^{\star}$ & 19.69 & $\begin{array}{l}{[\mathrm{M}+} \\
\mathrm{H}]^{+}\end{array}$ & 433.1122 & 433.1129 & -1.577 & $\mathrm{C}_{21} \mathrm{H}_{20} \mathrm{O}_{10}$ & Isovitexin & $397.0962 ; 313.0708 ; 283.05$ \\
\hline $156^{\star}$ & 19.70 & $\begin{array}{l}{[\mathrm{M}+} \\
\mathrm{H}]^{+}\end{array}$ & 465.1026 & 465.1028 & -0.328 & $\mathrm{C}_{21} \mathrm{H}_{20} \mathrm{O}_{12}$ & Hyperin & $465.1013 ; 303.0503 ; 169.01$ \\
\hline 157 & 19.73 & {$[\mathrm{M}-\mathrm{H}]^{-}$} & 771.2359 & 771.2342 & 2.178 & $\mathrm{C}_{34} \mathrm{H}_{44} \mathrm{O}_{20}$ & Alpha-glucosyl hesperidin & $771.2362 ; 301.0737$ \\
\hline $158^{\star}$ & 19.74 & {$[\mathrm{M}-\mathrm{H}]^{-}$} & 515.1193 & 515.1184 & 1.781 & $\mathrm{C}_{25} \mathrm{H}_{24} \mathrm{O}_{12}$ & Isochlorogenic acid A & 353.0903; 191.0578; 135.04 \\
\hline 159 & 19.75 & $\begin{array}{l}{[\mathrm{M}+} \\
\mathrm{H}]^{+}\end{array}$ & 449.1077 & 449.1078 & -0.307 & $\mathrm{C}_{21} \mathrm{H}_{20} \mathrm{O}_{11}$ & Kaempferol-3-O-glucoside & $449.1073 ; 287.0536$ \\
\hline 160 & 19.77 & $\begin{array}{l}{[\mathrm{M}+} \\
\mathrm{H}]^{+}\end{array}$ & 463.1229 & 463.1235 & -1.356 & $\mathrm{C}_{22} \mathrm{H}_{22} \mathrm{O}_{11}$ & Tectorigenin 4'-glucoside & $301.0773 ; 286.0538$ \\
\hline 161 & 19.84 & {$[\mathrm{M}-\mathrm{H}]-$} & 341.1243 & 341.1231 & 3.623 & $\mathrm{C}_{16} \mathrm{H}_{22} \mathrm{O}_{8}$ & Coniferin & 179.0587 \\
\hline 162 & 19.98 & {$[\mathrm{M}-\mathrm{H}]^{-}$} & 577.1567 & 577.1552 & 2.700 & $\mathrm{C}_{27} \mathrm{H}_{30} \mathrm{O}_{14}$ & Isoviolanthin & $577.1571 ; 413.0846 ; 293.04$ \\
\hline $163^{\star}$ & 19.99 & {$[\mathrm{M}-\mathrm{H}]^{-}$} & 461.0728 & 461.0715 & 2.880 & $\mathrm{C}_{21} \mathrm{H}_{18} \mathrm{O}_{12}$ & Scutellarin & $461.0726 ; 285.0405$ \\
\hline $164^{\star}$ & 20.00 & $\begin{array}{l}{[\mathrm{M}+} \\
\mathrm{H}]^{+}\end{array}$ & 303.0494 & 303.0499 & -1.911 & $\mathrm{C}_{15} \mathrm{H}_{10} \mathrm{O}_{7}$ & Quercetin & $303.0488 ; 229.0475 ; 153.01$ \\
\hline $165^{\star}$ & 20.00 & $\begin{array}{l}{[\mathrm{M}+} \\
\mathrm{H}]^{+}\end{array}$ & 465.1023 & 465.1028 & -1.059 & $\mathrm{C}_{21} \mathrm{H}_{20} \mathrm{O}_{12}$ & Isoquercitrin & $465.1026 ; 303.0481 ; 289.06$ \\
\hline
\end{tabular}




\begin{tabular}{|c|c|c|c|c|c|c|c|c|}
\hline No. & $\begin{array}{l}\mathrm{RT} \\
/ \mathrm{min}\end{array}$ & $\begin{array}{l}\text { lon } \\
\text { model }\end{array}$ & $\begin{array}{l}\text { Measured } \\
\text { mass /Da }\end{array}$ & $\begin{array}{l}\text { Calculated } \\
\text { mass /Da }\end{array}$ & $\begin{array}{l}\text { Error } \\
\text { /ppm }\end{array}$ & $\begin{array}{l}\text { Molecular } \\
\text { formula }\end{array}$ & Identification & Fragment ions \\
\hline 166 & 20.01 & {$[\mathrm{M}-\mathrm{H}]^{-}$} & 609.1466 & 609.1450 & 2.625 & $\mathrm{C}_{27} \mathrm{H}_{30} \mathrm{O}_{16}$ & Rutin & $\begin{array}{l}609.1458 ; 301.0358 ; 271.02 \\
255.0320\end{array}$ \\
\hline 167 & 20.02 & $\begin{array}{l}{[\mathrm{M}+} \\
\mathrm{H}]^{+}\end{array}$ & 611.1598 & 611.1607 & -1.425 & $\mathrm{C}_{27} \mathrm{H}_{30} \mathrm{O}_{16}$ & Lucenin-2 & $557.1270 ; 353.0646 ; 473.09$ \\
\hline $168^{*}$ & 20.05 & {$[\mathrm{M}-\mathrm{H}]^{-}$} & 623.1984 & 623.1970 & 2.107 & $\mathrm{C}_{29} \mathrm{H}_{36} \mathrm{O}_{15}$ & Verbascoside & $\begin{array}{l}\text { 623.2017; } 461.1658 ; 179.03 \\
161.0240 ; 135.0455\end{array}$ \\
\hline 169 & 20.06 & $\begin{array}{l}{[\mathrm{M}+} \\
\mathrm{H}]^{+}\end{array}$ & 493.1336 & 493.1341 & -0.958 & $\mathrm{C}_{23} \mathrm{H}_{24} \mathrm{O}_{12}$ & Iristectorin B & $\begin{array}{l}343.0798 ; 331.0802 ; 316.05 \\
301.0328\end{array}$ \\
\hline 170 & 20.09 & {$[\mathrm{M}-\mathrm{H}]^{-}$} & 433.1140 & 433.1129 & 2.090 & $\mathrm{C}_{21} \mathrm{H}_{22} \mathrm{O}_{10}$ & Chalconaringenin 4-0-glucoside & $\begin{array}{l}271.0599 ; 177.0182 ; 151.00 \\
119.0492\end{array}$ \\
\hline $171^{*}$ & 20.20 & $\begin{array}{l}{[\mathrm{M}+} \\
\mathrm{H}]^{+}\end{array}$ & 229.0856 & 229.0859 & -1.269 & $\mathrm{C}_{14} \mathrm{H}_{12} \mathrm{O}_{3}$ & Resveratrol & $152.061 ; 107.0472 ; 91.0527$ \\
\hline 172 & 20.21 & {$[\mathrm{M}-\mathrm{H}]-$} & 597.1828 & 597.1814 & 2.350 & $\mathrm{C}_{27} \mathrm{H}_{34} \mathrm{O}_{15}$ & Phloretin-3',5'-di-C-glucoside & $387.1080 ; 357.0981$ \\
\hline 173 & 20.27 & $\stackrel{[M+}{\mathrm{H}]+}$ & 581.1857 & 581.1865 & -1.397 & $\mathrm{C}_{27} \mathrm{H}_{32} \mathrm{O}_{14}$ & Isoliquiritigenin-4,4'-diglucoside & 257.0854 \\
\hline 174 & 20.31 & {$[\mathrm{M}-\mathrm{H}]^{-}$} & 579.1725 & 579.1708 & 2.700 & $\mathrm{C}_{27} \mathrm{H}_{32} \mathrm{O}_{14}$ & Narirutin & $\begin{array}{l}579.1727 ; 543.2543 ; 295.06 \\
271.0613 ; 151.0020\end{array}$ \\
\hline 175 & 20.43 & $\begin{array}{l}{[\mathrm{M}+} \\
\mathrm{H}]^{+}\end{array}$ & 465.1025 & 465.1028 & -0.521 & $\mathrm{C}_{21} \mathrm{H}_{20} \mathrm{O}_{12}$ & $\begin{array}{l}\text { Quercetin-4-O- } \beta \text {-D-glucoside,Herbacetin-7- } \\
O-\beta \text {-D-glucoside }\end{array}$ & $465.0985 ; 303.0488$ \\
\hline 176 & 20.57 & {$[\mathrm{M}-\mathrm{H}]^{-}$} & 547.1459 & 547.1446 & 2.290 & $\mathrm{C}_{26} \mathrm{H}_{28} \mathrm{O}_{13}$ & Chrysin 6-CPen-8-CHex & 547.1432 \\
\hline 177 & 20.59 & {$[\mathrm{M}-\mathrm{H}]^{-}$} & 505.0999 & 505.0977 & -2.415 & $\mathrm{C}_{23} \mathrm{H}_{22} \mathrm{O}_{13}$ & Viscidulin li 2'-O- $\beta$-D-glucuronide & $\begin{array}{l}505.1091 ; 329.0665 ; 314.04 \\
299.0170\end{array}$ \\
\hline 178 & 20.66 & $\begin{array}{l}{[\mathrm{M}+} \\
\mathrm{H}]^{+}\end{array}$ & 563.1754 & 563.1759 & -0.883 & $\mathrm{C}_{27} \mathrm{H}_{30} \mathrm{O}_{13}$ & Glycyroside & $269.0824 ; 254.0583$ \\
\hline 179 & 20.69 & {$[\mathrm{M}-\mathrm{H}]^{-}$} & 459.1298 & 459.1286 & 2.650 & $\mathrm{C}_{23} \mathrm{H}_{24} \mathrm{O}_{10}$ & 6'-Acetyliquiritin & $459.1308 ; 255.0659 ; 135.00$ \\
\hline $180^{*}$ & 20.70 & {$[\mathrm{M}-\mathrm{H}]^{-}$} & 301.0718 & 301.0707 & 3.804 & $\mathrm{C}_{16} \mathrm{H}_{14} \mathrm{O}_{6}$ & Hesperetin & $\begin{array}{l}301.0682 ; 286.0490 ; 242.06 \\
215.0487 ; 163.1426 ; 164.01\end{array}$ \\
\hline $181^{*}$ & 20.78 & {$[\mathrm{M}-\mathrm{H}]^{-}$} & 577.1566 & 579.1708 & 2.388 & $\mathrm{C}_{27} \mathrm{H}_{32} \mathrm{O}_{14}$ & Naringin & $\begin{array}{l}579.1759 ; 459.1184 ; 313.07 \\
271.0620 ; 151.0046\end{array}$ \\
\hline $182^{*}$ & 20.82 & {$[\mathrm{M}-\mathrm{H}]^{-}$} & 623.1985 & 623.1970 & 2.316 & $\mathrm{C}_{29} \mathrm{H}_{36} \mathrm{O}_{15}$ & Isoaceteoside & 623.2040; 461.1658; 161.02 \\
\hline 183 & 20.83 & $\begin{array}{l}{[\mathrm{M}+} \\
\mathrm{H}]^{+}\end{array}$ & 249.1483 & 249.1485 & -0.887 & $\mathrm{C}_{15} \mathrm{H}_{20} \mathrm{O}_{3}$ & 4-Shogaol & $137.0617 ; 122.0376 ; 94.042$ \\
\hline 184 & 20.88 & {$[\mathrm{M}-\mathrm{H}]^{-}$} & 581.1885 & 581.1865 & 3.541 & $\mathrm{C}_{27} \mathrm{H}_{34} \mathrm{O}_{14}$ & $\begin{array}{l}2,4,6-\text { Trihydroxydihydrochalcone-3 - } C \text { - } \\
\beta \text {-D-glucoside- } 6-O-\beta \text {-D-glucoside }\end{array}$ & $\begin{array}{l}581.1880 ; 461.1468 ; 329 \otimes 1 C \\
299.0928 ; 167.0358\end{array}$ \\
\hline 185 & 20.88 & {$[\mathrm{M}-\mathrm{H}]^{-}$} & 635.1622 & 635.1607 & 2.470 & $\mathrm{C}_{29} \mathrm{H}_{32} \mathrm{O}_{16}$ & Multiflorin A & $591.1745 ; 255.0658 ; 135.00$ \\
\hline 186 & 20.92 & {$[\mathrm{M}-\mathrm{H}]^{-}$} & 329.0669 & 329.0656 & 3.829 & $\mathrm{C}_{17} \mathrm{H}_{14} \mathrm{O}_{7}$ & Iristectorigenin A & $\begin{array}{l}314.0429 ; 299.0200 ; 282.01 \\
255.0319 ; 243.0316 ; 271.02 \\
227.0369 ; 215.0270 ; 199.04 \\
187.0426 ; 171.0471 ; 161.99 \\
134.0021 ;\end{array}$ \\
\hline 187 & 20.92 & $\begin{array}{l}{[\mathrm{M}+} \\
\mathrm{H}]^{+}\end{array}$ & 493.1336 & 493.1341 & -0.837 & $\mathrm{C}_{23} \mathrm{H}_{24} \mathrm{O}_{12}$ & Iristectorin A & $331.0797 ; 316.0564 ; 301.03$ \\
\hline 188 & 21.17 & {$[\mathrm{M}-\mathrm{H}]^{-}$} & 431.0985 & 431.0973 & 2.799 & $\mathrm{C}_{21} \mathrm{H}_{20} \mathrm{O}_{10}$ & kaempferol-3-rhamnoside & $255.0230 ; 227.0374$ \\
\hline $189 *$ & 21.19 & {$[\mathrm{M}-\mathrm{H}]^{-}$} & 609.1828 & 609.1814 & 2.304 & $\mathrm{C}_{28} \mathrm{H}_{34} \mathrm{O}_{15}$ & Hesperidin & $\begin{array}{l}609.1875 ; 343.0831 ; 325.07 \\
301.0728 ; 286.0496\end{array}$ \\
\hline 190 & 21.21 & $\begin{array}{l}{[\mathrm{M}+} \\
\mathrm{H}]^{+}\end{array}$ & 361.0911 & 361.0918 & -2.032 & $\mathrm{C}_{18} \mathrm{H}_{16} \mathrm{O}_{8}$ & Isoirigenin & $361.0854 ; 301.0707$ \\
\hline 191 & 21.21 & $\begin{array}{l}{[\mathrm{M}+} \\
\mathrm{H}]^{+}\end{array}$ & 523.1442 & 523.1446 & -0.740 & $\mathrm{C}_{24} \mathrm{H}_{26} \mathrm{O}_{13}$ & Iridin & $361.0922 ; 346.0675 ; 301.07$ \\
\hline 192 & 21.22 & {$[\mathrm{M}-\mathrm{H}]^{-}$} & 463.1250 & 463.1235 & 3.200 & $\mathrm{C}_{22} \mathrm{H}_{24} \mathrm{O}_{11}$ & Hesperetin-O-glucoside & $\begin{array}{l}301.0720 ; 286.0450 ; 240.05 \\
164.0105\end{array}$ \\
\hline
\end{tabular}




\begin{tabular}{|c|c|c|c|c|c|c|c|c|}
\hline No. & $\begin{array}{l}\mathrm{RT} \\
/ \mathrm{min}\end{array}$ & $\begin{array}{l}\text { lon } \\
\text { model }\end{array}$ & $\begin{array}{l}\text { Measured } \\
\text { mass /Da }\end{array}$ & $\begin{array}{l}\text { Calculated } \\
\text { mass /Da }\end{array}$ & $\begin{array}{l}\text { Error } \\
\text { /ppm }\end{array}$ & $\begin{array}{l}\text { Molecular } \\
\text { formula }\end{array}$ & Identification & Fragment ions \\
\hline \multirow[t]{2}{*}{193} & \multirow[t]{2}{*}{21.28} & \multirow[t]{2}{*}[\mathrm{M}-\mathrm{H}]{$^{-}$} & \multirow[t]{2}{*}{547.1460} & \multirow[t]{2}{*}{547.1446} & \multirow[t]{2}{*}{2.527} & \multirow[t]{2}{*}{$\mathrm{C}_{26} \mathrm{H}_{28} \mathrm{O}_{13}$} & Chrysin 6-C- $\beta$-D-glucoside- & \multirow{2}{*}{$\begin{array}{l}547.1465 ; 457.1157 ; 427.10 \\
367.0822 ; 337.0714\end{array}$} \\
\hline & & & & & & & 8-C- $\beta$-L-arabinoside & \\
\hline 194 & 21.38 & {$[\mathrm{M}-\mathrm{H}]^{-}$} & 161.0233 & 161.0233 & -0.071 & $\mathrm{C}_{9} \mathrm{H}_{6} \mathrm{O}_{3}$ & Folic acid & $91.0554 ; 62.0163$ \\
\hline $195^{\star}$ & 21.38 & {$[\mathrm{M}-\mathrm{H}]^{-}$} & 515.1194 & 515.1184 & 1.898 & $\mathrm{C}_{25} \mathrm{H}_{24} \mathrm{O}_{12}$ & Isochlorogenic acid C & $\begin{array}{l}515.1216 ; 353.0884 ; 191.05 \\
179.0345 ; 173.0453\end{array}$ \\
\hline $196^{*}$ & 21.51 & {$[\mathrm{M}-\mathrm{H}]^{-}$} & 447.0935 & 447.0922 & 3.024 & $\mathrm{C}_{21} \mathrm{H}_{20} \mathrm{O}_{11}$ & Astragalin & $\begin{array}{l}327.0475 ; 284.0347 ; 255.02 \\
227.0349 ;\end{array}$ \\
\hline 197 & 21.53 & {$[\mathrm{M}-\mathrm{H}]^{-}$} & 693.2769 & 693.2753 & 2.255 & $\mathrm{C}_{34} \mathrm{H}_{46} \mathrm{O}_{15}$ & Nominin-17- $\beta$-D-glucoside & $\begin{array}{l}693.2793 ; 565.2748 ; 517.15 \\
271.1715 ; 175.0416\end{array}$ \\
\hline 198 & 21.58 & $\begin{array}{l}{[\mathrm{M}+} \\
\mathrm{H}]^{+}\end{array}$ & 534.2108 & 534.2114 & -1.036 & $\mathrm{C}_{25} \mathrm{H}_{32} \mathrm{~N}_{5} \mathrm{O}_{6} \mathrm{Cl}$ & Astin N & $449.1598 ; 131.0482 ; 106.06$ \\
\hline $199 *$ & 21.59 & {$[\mathrm{M}-\mathrm{H}]^{-}$} & 609.1828 & 609.1814 & 2.304 & $\mathrm{C}_{28} \mathrm{H}_{34} \mathrm{O}_{15}$ & Neohesperidin & $609.1797 ; 301.0726$ \\
\hline 200 & 21.63 & {$[\mathrm{M}-\mathrm{H}]-$} & 813.2103 & 813.2084 & 2.375 & $\mathrm{C}_{35} \mathrm{H}_{42} \mathrm{O}_{22}$ & $\begin{array}{l}\text { Limocitrin-3-O-(5-a-glucosyl-HMG)- } \beta \text { - } \\
\text { glucoside }\end{array}$ & $651.1520 ; 345.0613$ \\
\hline 201 & 21.65 & $\begin{array}{l}{[\mathrm{M}+} \\
\mathrm{H}]+\end{array}$ & 579.1702 & 579.1708 & -1.039 & $\mathrm{C}_{27} \mathrm{H}_{30} \mathrm{O}_{14}$ & Violanthin & 417.1044 \\
\hline 202 & 21.68 & {$[\mathrm{M}-\mathrm{H}]^{-}$} & 547.1461 & 547.1446 & 2.637 & $\mathrm{C}_{26} \mathrm{H}_{28} \mathrm{O}_{13}$ & Chrysin 6-CHex-8-CPen & $\begin{array}{l}547.1501 ; 457.1147 ; 427.10 \\
367.0826 ; 337.0715\end{array}$ \\
\hline $203^{*}$ & 21.73 & $\begin{array}{l}{[\mathrm{M}+} \\
\mathrm{H}]^{+}\end{array}$ & 133.0647 & 133.0648 & -0.081 & $\mathrm{C}_{9} \mathrm{H}_{8} \mathrm{O}$ & Cinnamaldehyde & $115.0538 ; 105.0673 ; 86.100$ \\
\hline 204 & 21.83 & $\begin{array}{l}{[\mathrm{M}+} \\
\mathrm{H}]^{+}\end{array}$ & 845.2338 & 845.2346 & -1.010 & $\mathrm{C}_{36} \mathrm{H}_{44} \mathrm{O}_{23}$ & $\begin{array}{l}\text { limocitrol-3-O-( 5-a-glucosyl-HMG)- } \beta \text { - } \\
\text { glucoside }\end{array}$ & $377.0854 ; 362.0621$ \\
\hline $205^{\star}$ & 21.91 & {$[\mathrm{M}-\mathrm{H}]^{-}$} & 255.0662 & 255.0652 & 3.939 & $\mathrm{C}_{15} \mathrm{H}_{12} \mathrm{O}_{4}$ & Liquiritigenin & $\begin{array}{l}255.0708 ; 135.0090 ; 119.05 \\
91.0192\end{array}$ \\
\hline 206 & 21.95 & {$[\mathrm{M}-\mathrm{H}]^{-}$} & 623.1623 & 623.1607 & 2.614 & $\mathrm{C}_{28} \mathrm{H}_{32} \mathrm{O}_{16}$ & Isorhamnetin-3-O-rutinoside & $\begin{array}{l}623.1634 ; 461.1724 ; 315.04 \\
161.0253\end{array}$ \\
\hline 207 & 21.97 & {$[\mathrm{M}-\mathrm{H}]^{-}$} & 549.1617 & 549.1603 & 2.682 & $\mathrm{C}_{26} \mathrm{H}_{30} \mathrm{O}_{13}$ & Isoliquiritin Apioside & $549.1605 ; 255.0650 ; 135.00$ \\
\hline 208 & 22.01 & {$[\mathrm{M}-\mathrm{H}]^{-}$} & 463.1253 & 463.1235 & 3.934 & $\mathrm{C}_{22} \mathrm{H}_{24} \mathrm{O}_{11}$ & Hesperetin-O-glucoside & $\begin{array}{l}463.1230 ; 301.0712 ; 257.08 \\
164.0100\end{array}$ \\
\hline 209 & 22.05 & {$[\mathrm{M}-\mathrm{H}]^{-}$} & 201.0186 & 201.0182 & 1.964 & $\mathrm{C}_{11} \mathrm{H}_{6} \mathrm{O}_{4}$ & Xanthotoxol & $201.0196 ; 145.0314$ \\
\hline $210^{*}$ & 22.07 & {$[\mathrm{M}-\mathrm{H}]^{-}$} & 417.1191 & 417.1180 & 2.712 & $\mathrm{C}_{21} \mathrm{H}_{22} \mathrm{O}_{9}$ & Isoliquiritin & $\begin{array}{l}417.1149 ; 255.0695 ; 148.01 \\
135.0080\end{array}$ \\
\hline 211 & 22.17 & $\begin{array}{l}{[\mathrm{M}+} \\
\mathrm{H}]^{+}\end{array}$ & 609.1810 & 609.1814 & -0.602 & $\mathrm{C}_{28} \mathrm{H}_{32} \mathrm{O}_{15}$ & Diosmin or neodiosmin & $301.0697 ; 286.0462$ \\
\hline $212^{*}$ & 22.26 & $\begin{array}{l}{[\mathrm{M}+} \\
\mathrm{FA}-\mathrm{H}]^{-}\end{array}$ & 475.1249 & 475.1235 & 2.867 & $\mathrm{C}_{22} \mathrm{H}_{22} \mathrm{O}_{9}$ & Ononin & 267.252 \\
\hline 213 & 22.36 & {$[\mathrm{M}-\mathrm{H}]^{-}$} & 475.0884 & 475.0871 & 2.752 & $\mathrm{C}_{22} \mathrm{H}_{20} \mathrm{O}_{12}$ & $\begin{array}{l}5,7,2 \text {-Trihydroxy-6-methoxy flavone-7-0- } \\
\text { glucuronide }\end{array}$ & $475.0933 ; 299.0580 ; 284.03$ \\
\hline 214 & 22.39 & $\begin{array}{l}{[\mathrm{M}+} \\
\mathrm{H}]^{+}\end{array}$ & 532.2398 & 532.2402 & -0.826 & $\mathrm{C}_{25} \mathrm{H}_{33} \mathrm{~N}_{5} \mathrm{O}_{8}$ & Asterinin A & $\begin{array}{l}532.1802 ; 514.2322 ; 338.17 \\
235.1084 ; 131.0480\end{array}$ \\
\hline 215 & 22.48 & {$[\mathrm{M}-\mathrm{H}]^{-}$} & 353.1033 & 353.1020 & 3.782 & $\mathrm{C}_{20} \mathrm{H}_{18} \mathrm{O}_{6}$ & Licoisoflavone A & $\begin{array}{l}\text { 353.1060; } 227.0704 ; 125.02 \\
83.0125\end{array}$ \\
\hline 216 & 22.49 & {$[\mathrm{M}-\mathrm{H}]^{-}$} & 549.1616 & 549.1603 & 2.463 & $\mathrm{C}_{26} \mathrm{H}_{30} \mathrm{O}_{13}$ & Licuraside & $549.1569 ; 255.0698 ; 135.01$ \\
\hline 217 & 22.57 & {$[\mathrm{M}-\mathrm{H}]^{-}$} & 447.0935 & 447.0922 & 3.024 & $\mathrm{C}_{21} \mathrm{H}_{20} \mathrm{O}_{11}$ & Baicalein- 7-O- $\beta$-D-glucopyranoside & $\begin{array}{l}447.0950 ; 271.0612 ; 243.06 \\
225.0559\end{array}$ \\
\hline 218 & 22.57 & {$[\mathrm{M}-\mathrm{H}]^{-}$} & 651.1574 & 651.1556 & 2.725 & $\mathrm{C}_{29} \mathrm{H}_{32} \mathrm{O}_{17}$ & $\begin{array}{l}\text { 3',3,5-Hydroxy-4',5'-methoxyflavone-0- } \\
\text { glucoside-orhamnoside }\end{array}$ & $\begin{array}{l}651.1634 ; 589.1628 ; 549.13 \\
507.1189\end{array}$ \\
\hline 219 & 22.58 & $\begin{array}{l}{[\mathrm{M}+} \\
\mathrm{H}]^{+}\end{array}$ & 347.0756 & 347.0761 & -1.509 & $\mathrm{C}_{17} \mathrm{H}_{14} \mathrm{O}_{8}$ & Iristectorigenin B & $331.0797 ; 316.0574 ; 273.03$ \\
\hline 220 & 22.76 & $\begin{array}{l}{[\mathrm{M}+} \\
\mathrm{H}]^{+}\end{array}$ & 317.0652 & 317.0656 & -1.227 & $\mathrm{C}_{16} \mathrm{H}_{12} \mathrm{O}_{7}$ & Irilin D & $\begin{array}{l}317.0668 ; 302.0418 ; 245.04 \\
168.0044\end{array}$ \\
\hline
\end{tabular}




\begin{tabular}{|c|c|c|c|c|c|c|c|c|}
\hline No. & $\begin{array}{l}\mathrm{RT} \\
/ \mathrm{min}\end{array}$ & $\begin{array}{l}\text { lon } \\
\text { model }\end{array}$ & $\begin{array}{l}\text { Measured } \\
\text { mass /Da }\end{array}$ & $\begin{array}{l}\text { Calculated } \\
\text { mass /Da }\end{array}$ & $\begin{array}{l}\text { Error } \\
\text { /ppm }\end{array}$ & $\begin{array}{l}\text { Molecular } \\
\text { formula }\end{array}$ & Identification & Fragment ions \\
\hline 221 & 22.78 & {$[\mathrm{M}-\mathrm{H}]^{-}$} & 621.1465 & 621.1450 & 2.356 & $\mathrm{C}_{28} \mathrm{H}_{30} \mathrm{O}_{16}$ & Wogonin-7-O-glu-glu acid & $\begin{array}{l}621.1494 ; 445.1134 ; 430.09 \\
283.0600 ; 268.0349 ; 175.02\end{array}$ \\
\hline 222 & 22.87 & $\begin{array}{l}{[\mathrm{M}+} \\
\mathrm{H}]^{+}\end{array}$ & 586.1820 & 586.1830 & -1.655 & $\mathrm{C}_{25} \mathrm{H}_{33} \mathrm{~N}_{5} \mathrm{O}_{7} \mathrm{Cl}_{2}$ & Astin B isomer & $\begin{array}{l}586.1751 ; 501.1269 ; 336.04 \\
249.0186 ; 194.0147 ; 106.06\end{array}$ \\
\hline $223^{*}$ & 22.89 & $\begin{array}{l}{[\mathrm{M}+} \\
\mathrm{H}]^{+}\end{array}$ & 287.0544 & 287.0550 & -2.001 & $\mathrm{C}_{15} \mathrm{H}_{10} \mathrm{O}_{6}$ & Luteolin & $287.0548 ; 153.0192 ; 135.04$ \\
\hline $224 *$ & 22.90 & {$[\mathrm{M}-\mathrm{H}]^{-}$} & 445.0776 & 445.0765 & 2.274 & $\mathrm{C}_{21} \mathrm{H}_{18} \mathrm{O}_{11}$ & Baicalin & $445.0786 ; 269.0466$ \\
\hline 225 & 22.98 & {$[\mathrm{M}-\mathrm{H}]^{-}$} & 263.1289 & 263.1278 & 4.045 & $\mathrm{C}_{15} \mathrm{H}_{20} \mathrm{O}_{4}$ & (+)-Abscisic acid & $\begin{array}{l}\text { 219.1463; 204.1170; } 153.09 \\
\text { 122.0382; 109.0276; }\end{array}$ \\
\hline 226 & 23.05 & {$[\mathrm{M}-\mathrm{H}]-$} & 621.1829 & 621.1814 & 2.356 & $\mathrm{C}_{29} \mathrm{H}_{34} \mathrm{O}_{15}$ & Isocrenatoside or isomer & $203.0362 ; 179.0374 ; 161.02$ \\
\hline 227 & 23.07 & {$[\mathrm{M}-\mathrm{H}]-$} & 695.1989 & 695.1970 & 2.594 & $\mathrm{C}_{35} \mathrm{H}_{36} \mathrm{O}_{15}$ & Licorice-glycoside B/D1/D2 & $\begin{array}{l}549.1584 ; 531.1510 ; 255.06 \\
135.0104\end{array}$ \\
\hline 228 & 23.07 & {$[\mathrm{M}-\mathrm{H}]-$} & 725.2090 & 725.2076 & 1.887 & $\mathrm{C}_{36} \mathrm{H}_{38} \mathrm{O}_{16}$ & Licorice-glycoside A/C1/C2 & $549.1600 ; 531.1486 ; 255.06$ \\
\hline $229 *$ & 23.24 & {$[\mathrm{M}-\mathrm{H}]^{-}$} & 147.0439 & 147.0441 & -0.186 & $\mathrm{C}_{9} \mathrm{H}_{8} \mathrm{O}_{2}$ & Cinnamic acid & 103.0553 \\
\hline 230 & 23.47 & $\begin{array}{l}{[\mathrm{M}+} \\
\mathrm{H}]^{+}\end{array}$ & 586.1826 & 586.1830 & -0.615 & $\mathrm{C}_{25} \mathrm{H}_{33} \mathrm{~N}_{5} \mathrm{O}_{7} \mathrm{Cl}_{2}$ & Astin B & $\begin{array}{l}586.1946 ; 409.9996 ; 338.06 \\
251.0331 ; 106.0645\end{array}$ \\
\hline 231 & 23.59 & {$[\mathrm{M}-\mathrm{H}]^{-}$} & 591.1726 & 591.1708 & 3.008 & $\mathrm{C}_{28} \mathrm{H}_{32} \mathrm{O}_{14}$ & 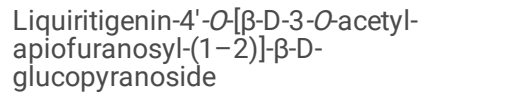 & $\begin{array}{l}591.1762 ; 471.1338 ; 403.10 \\
307.0604\end{array}$ \\
\hline 232 & 23.69 & {$[\mathrm{M}-\mathrm{H}]-$} & 711.2881 & 711.2859 & 3.133 & $\mathrm{C}_{34} \mathrm{H}_{48} \mathrm{O}_{16}$ & Nomilinic acid glucoside & $651.2662 ; 607.2740$ \\
\hline 233 & 23.70 & {$[\mathrm{M}-\mathrm{H}]^{-}$} & 505.0994 & 505.0977 & 3.372 & $\mathrm{C}_{23} \mathrm{H}_{22} \mathrm{O}_{13}$ & Viscidulin li 2'-O- $\beta$-D-glucuronide isomer & $\begin{array}{l}505.1024 ; 329.0651 ; 314.04 \\
299.0187\end{array}$ \\
\hline 234 & 23.72 & $\begin{array}{l}{[\mathrm{M}+} \\
\mathrm{H}]^{+}\end{array}$ & 534.2110 & 534.2114 & -0.726 & $\mathrm{C}_{25} \mathrm{H}_{32} \mathrm{~N}_{5} \mathrm{O}_{6} \mathrm{Cl}$ & Astin D & $\begin{array}{l}534.2099 ; 449.1561 ; 302.08 \\
215.0562\end{array}$ \\
\hline 235 & 23.82 & $\begin{array}{l}{[\mathrm{M}+} \\
\mathrm{H}]^{+}\end{array}$ & 179.0704 & 179.0703 & -1.288 & $\mathrm{C}_{10} \mathrm{H}_{10} \mathrm{O}_{3}$ & 2-Methoxycinnamic acid & $118.0416 ; 90.0466 ; 77.0388$ \\
\hline 236 & 23.88 & {$[\mathrm{M}-\mathrm{H}]^{-}$} & 445.0777 & 445.0765 & 2.746 & $\mathrm{C}_{21} \mathrm{H}_{18} \mathrm{O}_{11}$ & Norwogonin 7-O- $\beta$-D-glucuronide & $445.0811 ; 269.0464$ \\
\hline $237 *$ & 23.92 & {$[\mathrm{M}-\mathrm{H}]^{-}$} & 271.0613 & 271.0601 & 4.243 & $\mathrm{C}_{15} \mathrm{H}_{12} \mathrm{O}_{5}$ & Naringenin & 151.001 \\
\hline 238 & 23.92 & {$[\mathrm{M}-\mathrm{H}]^{-}$} & 753.2254 & 753.2237 & 2.316 & $\mathrm{C}_{34} \mathrm{H}_{42} \mathrm{O}_{19}$ & Brutieridin & $\begin{array}{l}\text { 753.2278; } 691.2259 ; 651.19 \\
609.1844 ; 301.0733\end{array}$ \\
\hline 239 & 24.05 & $\begin{array}{l}{[\mathrm{M}+} \\
\mathrm{H}]^{+}\end{array}$ & 163.0752 & 163.0754 & -0.206 & $\mathrm{C}_{10} \mathrm{H}_{10} \mathrm{O}_{2}$ & 2-Methoxycinnamaldehyde & $\begin{array}{l}145.0659 ; 131.0496 ; 115.05 \\
105.0697 ; 91.0533 ; 77.0383\end{array}$ \\
\hline $240 *$ & 24.07 & $\begin{array}{l}{[\mathrm{M}+} \\
\mathrm{H}]^{+}\end{array}$ & 133.0647 & 133.0648 & -0.051 & $\mathrm{C}_{9} \mathrm{H}_{8} \mathrm{O}$ & Cinnamaldehyde isomer & $133.0644 ; 115.0538$ \\
\hline 241 & 24.14 & $\begin{array}{l}{[\mathrm{M}+} \\
\mathrm{H}]^{+}\end{array}$ & 570.1876 & 570.1881 & -0.887 & $\mathrm{C}_{25} \mathrm{H}_{33} \mathrm{~N}_{5} \mathrm{O}_{6} \mathrm{Cl}_{2}$ & Astin C & $\begin{array}{l}570.1876 ; 485.1351 ; 337.08 \\
251.0333 ; 223.0388\end{array}$ \\
\hline 242 & 24.14 & $\begin{array}{l}{[\mathrm{M}+} \\
\mathrm{H}]^{+}\end{array}$ & 595.1696 & 595.1657 & 6.407 & $\mathrm{C}_{27} \mathrm{H}_{30} \mathrm{O}_{15}$ & Luteolin-7-O-rutinoside & $449.1167 ; 287.0527$ \\
\hline 243 & 24.28 & $\begin{array}{l}{[\mathrm{M}+} \\
\mathrm{H}]^{+}\end{array}$ & 516.2448 & 516.2453 & -0.900 & $\mathrm{C}_{25} \mathrm{H}_{33} \mathrm{~N}_{5} \mathrm{O}_{7}$ & Astin J & $\begin{array}{l}498.2315 ; 338.1695 ; 235.10 \\
218.0802 ; 193.0955 ; 151.08 \\
131.0477\end{array}$ \\
\hline 244 & 24.31 & {$[\mathrm{M}-\mathrm{H}]^{-}$} & 429.0827 & 429.0816 & 2.510 & $\mathrm{C}_{21} \mathrm{H}_{18} \mathrm{O}_{10}$ & Chrysin7-O- $\beta$-D-glucuronide & $\begin{array}{l}429.0829 ; 253.0511 ; 175.02 \\
113.0249\end{array}$ \\
\hline 245 & 24.32 & $\begin{array}{l}{[\mathrm{M}+} \\
\mathrm{H}]^{+}\end{array}$ & 255.0645 & 255.0652 & -2.765 & $\mathrm{C}_{15} \mathrm{H}_{10} \mathrm{O}_{4}$ & Daidzein & 137.0245 \\
\hline 246 & 24.32 & $\begin{array}{l}{[\mathrm{M}+} \\
\mathrm{H}]^{+}\end{array}$ & 471.2010 & 471.2013 & -0.752 & $\mathrm{C}_{26} \mathrm{H}_{30} \mathrm{O}_{8}$ & Limonin & $\begin{array}{l}471.2020 ; 453.1931 ; 425.19 \\
367.1914 ; 213.0920 ; 161.05\end{array}$ \\
\hline 247 & 24.34 & {$[\mathrm{M}-\mathrm{H}]-$} & 593.1877 & 593.1865 & 2.020 & $\mathrm{C}_{28} \mathrm{H}_{34} \mathrm{O}_{14}$ & Didymin & $\begin{array}{l}327.0881 ; 285.0785 ; 241.08 \\
164.0130\end{array}$ \\
\hline 248 & 24.40 & {$[\mathrm{M}-\mathrm{H}]^{-}$} & 473.0726 & 473.0715 & 2.363 & $\mathrm{C}_{22} \mathrm{H}_{18} \mathrm{O}_{12}$ & Cichoric acid & 149.0123 \\
\hline
\end{tabular}




\begin{tabular}{|c|c|c|c|c|c|c|c|c|}
\hline No. & $\begin{array}{l}\mathrm{RT} \\
\text { /min }\end{array}$ & $\begin{array}{l}\text { lon } \\
\text { model }\end{array}$ & $\begin{array}{l}\text { Measured } \\
\text { mass /Da }\end{array}$ & $\begin{array}{l}\text { Calculated } \\
\text { mass /Da }\end{array}$ & $\begin{array}{l}\text { Error } \\
\text { /ppm }\end{array}$ & $\begin{array}{l}\text { Molecular } \\
\text { formula }\end{array}$ & Identification & Fragment ions \\
\hline 249 & 24.40 & {$[\mathrm{M}-\mathrm{H}]^{-}$} & 475.0882 & 475.0871 & 2.374 & $\mathrm{C}_{22} \mathrm{H}_{20} \mathrm{O}_{12}$ & $\begin{array}{l}\text { 5,7,8-Trihydroxy-6-methoxy flavone-7-0- } \\
\text { glucuronide }\end{array}$ & $475.0954 ; 299.0567 ; 284.03$ \\
\hline \multirow[t]{2}{*}{250} & \multirow[t]{2}{*}{24.40} & \multirow[t]{2}{*}[\mathrm{M}-\mathrm{H}]{$^{-}$} & \multirow[t]{2}{*}{547.1456} & \multirow[t]{2}{*}{547.1446} & \multirow[t]{2}{*}{1.851} & \multirow[t]{2}{*}{$\mathrm{C}_{26} \mathrm{H}_{28} \mathrm{O}_{13}$} & & \multirow{2}{*}{$\begin{array}{l}547.1488 ; 457.1156 ; 427.10 \\
367.0826 ; 337.0722\end{array}$} \\
\hline & & & & & & & 8-C-a-L-arabinoside & \\
\hline 251 & 24.44 & {$[\mathrm{M}-\mathrm{H}]^{-}$} & 445.1142 & 445.1129 & 2.936 & $\mathrm{C}_{22} \mathrm{H}_{22} \mathrm{O}_{10}$ & Wogonin-5-O- $\beta-D$-glucoside & $\begin{array}{l}445.1167 ; 430.0944 ; 283.06 \\
267.0312 ; 239.0361\end{array}$ \\
\hline 252 & 24.48 & {$[\mathrm{M}-\mathrm{H}]^{-}$} & 461.1094 & 461.1078 & 3.410 & $\mathrm{C}_{22} \mathrm{H}_{22} \mathrm{O}_{11}$ & $\begin{array}{l}\text { (2S)-5,7-Dihydroxy-6-methoxyflavanone7- } \\
O-\beta \text {-D-glucuronide }\end{array}$ & $\begin{array}{l}\text { 461.1128; 285.0775; } 270.05 \\
165.9913 ; 113.0255\end{array}$ \\
\hline $253^{*}$ & 24.50 & {$[\mathrm{M}-\mathrm{H}]^{-}$} & 285.0768 & 285.0758 & 3.718 & $\mathrm{C}_{16} \mathrm{H}_{14} \mathrm{O}_{5}$ & Licochalcone B & $\begin{array}{l}285.0674 ; 270.0507 ; 177.01 \\
150.0310 ; 121.0272\end{array}$ \\
\hline 254 & 24.5 & {$[\mathrm{M}-\mathrm{H}]-$} & 635.1627 & 635.1607 & 3.241 & $\mathrm{C}_{29} \mathrm{H}_{36} \mathrm{O}_{16}$ & Campneoside II or its isomer & 635.1625 \\
\hline 255 & 24.67 & {$[\mathrm{M}-\mathrm{H}]^{-}$} & 459.0936 & 459.0922 & 3.076 & $\mathrm{C}_{22} \mathrm{H}_{20} \mathrm{O}_{11}$ & Oroxylin A 7-0-glucuronide & $459.0970 ; 283.0621 ; 268.03$ \\
\hline 256 & 24.7 & $\begin{array}{l}{[\mathrm{M}+} \\
\mathrm{H}]^{+}\end{array}$ & 219.1013 & 219.1016 & -1.099 & $\mathrm{C}_{13} \mathrm{H}_{14} \mathrm{O}_{3}$ & 6-Acetyl-2,2-dimethylchroman-4-one & $163.0376 ; 107.0491 ; 77.038$ \\
\hline 257 & 24.75 & {$[\mathrm{M}-\mathrm{H}]-$} & 299.0561 & 299.0550 & 3.697 & $\mathrm{C}_{16} \mathrm{H}_{12} \mathrm{O}_{6}$ & $\begin{array}{l}\text { 7,2',4'-Trihydroxy-5-methoxy-3- } \\
\text { arylcoumarin }\end{array}$ & $255.0323 ; 227.0373$ \\
\hline $258^{*}$ & 24.77 & {$[\mathrm{M}-\mathrm{H}]-$} & 269.0820 & 269.0808 & 4.440 & $\mathrm{C}_{16} \mathrm{H}_{14} \mathrm{O}_{4}$ & Echinatin & $\begin{array}{l}237.0611 ; 183.0856 ; 161.01 \\
133.0308 ; 120.0216 ; 92.026\end{array}$ \\
\hline 259 & 24.77 & $\begin{array}{l}{[\mathrm{M}+} \\
\mathrm{H}]^{+}\end{array}$ & 301.0718 & 301.0707 & 3.605 & $\mathrm{C}_{16} \mathrm{H}_{12} \mathrm{O}_{6}$ & Tectorigenin & $301.0700 ; 286.0462 ; 168.00$ \\
\hline 260 & 24.77 & {$[\mathrm{M}-\mathrm{H}]-$} & 301.0718 & 301.0707 & 4.004 & $\mathrm{C}_{16} \mathrm{H}_{14} \mathrm{O}_{6}$ & Tetrahydroxymethoxychalcone & $149.9976 ; 108.0216$ \\
\hline 261 & 24.77 & {$[\mathrm{M}-\mathrm{H}]^{-}$} & 445.0778 & 445.0765 & 2.881 & $\mathrm{C}_{21} \mathrm{H}_{18} \mathrm{O}_{11}$ & Norwogonin 8-O- $\beta$-D-glucuronide & $445.0785 ; 269.0454$ \\
\hline 262 & 24.88 & {$[\mathrm{M}-\mathrm{H}]^{-}$} & 475.0883 & 475.0871 & 2.500 & $\mathrm{C}_{22} \mathrm{H}_{20} \mathrm{O}_{12}$ & $\begin{array}{l}\text { 5,6,7-Trihydroxy-8-methoxyflavone-7-0- } \\
\text { glucuronopyranoside }\end{array}$ & $475.0899 ; 299.0555 ; 284.03$ \\
\hline $263^{*}$ & 24.90 & $\begin{array}{l}{[\mathrm{M}+} \\
\mathrm{FA}-\mathrm{H}]^{-}\end{array}$ & 491.1196 & 491.1184 & 2.377 & $\mathrm{C}_{22} \mathrm{H}_{22} \mathrm{O}_{10}$ & Tilianin & $491.2810 ; 283.0620 ; 268.04$ \\
\hline 264 & 24.9 & $\begin{array}{l}{[\mathrm{M}+} \\
\mathrm{FA}-\mathrm{H}]^{-}\end{array}$ & 491.1196 & 491.1184 & 2.377 & $\mathrm{C}_{22} \mathrm{H}_{22} \mathrm{O}_{10}$ & Trifolirhizin & $\begin{array}{l}283.0626 ; 268.0397 ; 239.03 \\
211.0431\end{array}$ \\
\hline 265 & 24.94 & {$[\mathrm{M}-\mathrm{H}]-$} & 857.2365 & 857.2346 & 2.212 & $\mathrm{C}_{37} \mathrm{H}_{46} \mathrm{O}_{23}$ & $\begin{array}{l}\text { 7,4-Dihydroxy-5,6,8,3- } \\
\text { tetramethoxyflavonol-3-0- }\end{array}$ & 593.1484; 551.1380; 389.08 \\
\hline & & & & & & & ( 5-a-glucosyl-HMG)- $\beta$-glucoside & \\
\hline $266^{*}$ & 25.07 & {$[\mathrm{M}-\mathrm{H}]^{-}$} & 459.0935 & 459.0922 & 2.815 & $\mathrm{C}_{22} \mathrm{H}_{20} \mathrm{O}_{11}$ & Wogonoside & $459.1119 ; 283.0631 ; 268.03$ \\
\hline 267 & 25.43 & $\begin{array}{l}{[\mathrm{M}+} \\
\mathrm{Na}]^{+}\end{array}$ & 231.0624 & 231.0628 & -1.472 & $\mathrm{C}_{11} \mathrm{H}_{12} \mathrm{O}_{4}$ & Ethyl Caffeate & $153.0172 ; 77.0380$ \\
\hline $268^{*}$ & 25.44 & $\begin{array}{l}{[\mathrm{M}+} \\
\mathrm{H}]^{+}\end{array}$ & 287.0545 & 287.0550 & -1.729 & $\mathrm{C}_{15} \mathrm{H}_{10} \mathrm{O}_{6}$ & Kaempferol & $287.0548 ; 231.0650 ; 153.01$ \\
\hline 269 & 25.51 & $\begin{array}{l}{[\mathrm{M}+} \\
\mathrm{H}]^{+}\end{array}$ & 331.0807 & 331.0812 & -1.719 & $\mathrm{C}_{17} \mathrm{H}_{14} \mathrm{O}_{7}$ & Iristectorigenin A & $\begin{array}{l}316.0639 ; 301.0407 ; 273.04 \\
245.0494\end{array}$ \\
\hline 270 & 25.56 & {$[\mathrm{M}-\mathrm{H}]^{-}$} & 445.0779 & 445.0765 & 2.960 & $\mathrm{C}_{21} \mathrm{H}_{18} \mathrm{O}_{11}$ & Baicalein $6-O-\beta$-D-glucuronide & $445.0811 ; 269.0450$ \\
\hline 271 & 25.59 & $\begin{array}{l}{[\mathrm{M}+} \\
\mathrm{H}]^{+}\end{array}$ & 221.1898 & 221.1900 & -1.049 & $\mathrm{C}_{15} \mathrm{H}_{24} \mathrm{O}$ & Alismol & $\begin{array}{l}\text { 105.0695; } 91.0538 ; 79.0573 \\
67.0542 ; 119.0842\end{array}$ \\
\hline 272 & 25.62 & $\begin{array}{l}{[\mathrm{M}+} \\
\mathrm{H}]^{+}\end{array}$ & 361.0920 & 361.0918 & -1.672 & $\mathrm{C}_{18} \mathrm{H}_{16} \mathrm{O}_{8}$ & Irigenin & $\begin{array}{l}361.0909 ; 331.0449 ; 232.03 \\
169.0131\end{array}$ \\
\hline $273^{*}$ & 25.63 & {$[\mathrm{M}-\mathrm{H}]^{-}$} & 359.0776 & 359.0761 & -3.450 & $\mathrm{C}_{18} \mathrm{H}_{16} \mathrm{O}_{8}$ & Rosmarinic acid & $\begin{array}{l}359.0774 ; 197.0469 ; 179.03 \\
161.0244 ; 135.0468 ; 123.04\end{array}$ \\
\hline $274^{*}$ & 25.73 & {$[\mathrm{M}-\mathrm{H}]^{-}$} & 269.0456 & 269.0444 & 4.349 & $\mathrm{C}_{15} \mathrm{H}_{10} \mathrm{O}_{5}$ & Norwogonin & $269.0458 ; 232.8405 ; 78.959$ \\
\hline 275 & 25.88 & {$[\mathrm{M}-\mathrm{H}]^{-}$} & 671.1628 & 671.1607 & 3.246 & $\mathrm{C}_{32} \mathrm{H}_{32} \mathrm{O}_{16}$ & 6"-O-Vanilloyliridin & $359.0766 ; 344.0538$ \\
\hline 276 & 25.91 & $\begin{array}{l}{[\mathrm{M}+} \\
\mathrm{H}]^{+}\end{array}$ & 711.2124 & 711.2131 & -0.971 & $\mathrm{C}_{32} \mathrm{H}_{38} \mathrm{O}_{18}$ & $\begin{array}{l}\text { monohydroxypentamethoxyflavonol-3-O- } \\
\text { HMG- } \beta \text {-glucoside }\end{array}$ & $405.1159 ; 390.0933 ; 375.06$ \\
\hline
\end{tabular}




\begin{tabular}{|c|c|c|c|c|c|c|c|c|}
\hline No. & $\begin{array}{l}\mathrm{RT} \\
/ \mathrm{min}\end{array}$ & $\begin{array}{l}\text { lon } \\
\text { model }\end{array}$ & $\begin{array}{l}\text { Measured } \\
\text { mass /Da }\end{array}$ & $\begin{array}{l}\text { Calculated } \\
\text { mass /Da }\end{array}$ & $\begin{array}{l}\text { Error } \\
\text { /ppm }\end{array}$ & $\begin{array}{l}\text { Molecular } \\
\text { formula }\end{array}$ & Identification & Fragment ions \\
\hline 277 & 25.93 & $\begin{array}{l}{[\mathrm{M}+} \\
\mathrm{H}]^{+}\end{array}$ & 547.3603 & 547.3629 & -4.824 & $\mathrm{C}_{32} \mathrm{H}_{50} \mathrm{O}_{7}$ & 16-Oxo-alisol A-24-acetate & $\begin{array}{l}\text { 487.3420; 469.3346; } 451.32 \\
415.2827 ; 189.1615\end{array}$ \\
\hline 278 & 25.94 & {$[\mathrm{M}-\mathrm{H}]^{-}$} & 547.1479 & 547.1446 & -0.347 & $\mathrm{C}_{26} \mathrm{H}_{28} \mathrm{O}_{13}$ & Chrysin-6-CHex-8-CPen & $\begin{array}{l}547.1469 ; 457.1168 ; 427.10 \\
367.0839 ; 337.0699\end{array}$ \\
\hline 279 & 25.98 & $\begin{array}{l}{[\mathrm{M}+} \\
\mathrm{FA}-\mathrm{H}]^{-}\end{array}$ & 533.1302 & 533.1290 & 2.294 & $\mathrm{C}_{24} \mathrm{H}_{24} \mathrm{O}_{11}$ & Agastachoside & $487.1302 ; 283.0624 ; 268.03$ \\
\hline 280 & 26.00 & {$[\mathrm{M}-\mathrm{H}]^{-}$} & 345.0617 & 345.0605 & 3.612 & $\mathrm{C}_{17} \mathrm{H}_{14} \mathrm{O}_{8}$ & Viscidulin lii & $\begin{array}{l}345.0624 ; 330.0392 ; 315.01 \\
287 ; 369\end{array}$ \\
\hline 281 & 26.00 & {$[\mathrm{M}-\mathrm{H}]^{-}$} & 489.1042 & 489.1028 & 2.939 & $\mathrm{C}_{23} \mathrm{H}_{22} \mathrm{O}_{12}$ & $\begin{array}{l}\text { 5,7-Dihydroxy-8,2'-dimethoxyflavone7- } 0-\beta \\
\text {-D-glucuronide }\end{array}$ & $\begin{array}{l}\text { 489.1039; } 313.0726 ; 289.04 \\
283.0255\end{array}$ \\
\hline $282^{*}$ & 26.10 & {$[\mathrm{M}-\mathrm{H}]^{-}$} & 255.0663 & 255.0652 & 4.370 & $\mathrm{C}_{15} \mathrm{H}_{12} \mathrm{O}_{4}$ & Isoliquiritigenin & $255.0678 ; 119.0499 ; 91.018$ \\
\hline 283 & 26.18 & $\begin{array}{l}{[\mathrm{M}+} \\
\mathrm{H}]^{+}\end{array}$ & 887.2806 & 887.2816 & -1.109 & $\mathrm{C}_{39} \mathrm{H}_{50} \mathrm{O}_{23}$ & $\begin{array}{l}\text { Natsudaidain-3-O-( 5-a-glucosyl-HMG)- } \beta \text { - } \\
\text { glucoside }\end{array}$ & $419.1345 ; 404.1107 ; 389.08$ \\
\hline 284 & 26.22 & $\begin{array}{l}{[\mathrm{M}+} \\
\mathrm{H}]^{+}\end{array}$ & 728.3973 & 728.3978 & -0.649 & $\mathrm{C}_{36} \mathrm{H}_{53} \mathrm{~N}_{7} \mathrm{O}_{9}$ & Citrusin III & $\begin{array}{l}710.3892 ; 700.4052 ; 587.31 \\
474.2331 ; 138.1269\end{array}$ \\
\hline $285^{\star}$ & 26.79 & {$[\mathrm{M}-\mathrm{H}]^{-}$} & 269.0458 & 269.0444 & 4.832 & $\mathrm{C}_{15} \mathrm{H}_{10} \mathrm{O}_{5}$ & Baicalein & $269.0455 ; 241.04564 ; 223.0$ \\
\hline 286 & 26.85 & {$[\mathrm{M}-\mathrm{H}]^{-}$} & 243.1026 & 243.1016 & 4.110 & $\mathrm{C}_{15} \mathrm{H}_{16} \mathrm{O}_{3}$ & 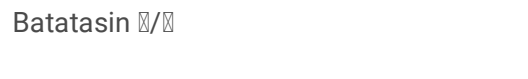 & $\begin{array}{l}243.1027 ; 137.0610 ; 122.03 \\
116.9939 ; 78.9609\end{array}$ \\
\hline $287 *$ & 27.09 & {$[\mathrm{M}-\mathrm{H}]^{-}$} & 267.0664 & 267.0652 & 4.586 & $\mathrm{C}_{16} \mathrm{H}_{12} \mathrm{O}_{4}$ & Formononetin & $267.0683 ; 223.0434 ; 178.99$ \\
\hline 288 & 27.10 & $\begin{array}{l}{[\mathrm{M}+} \\
\mathrm{H}]^{+}\end{array}$ & 373.1279 & 373.1282 & -1.070 & $\mathrm{C}_{20} \mathrm{H}_{20} \mathrm{O}_{7}$ & Isosinensetin & $373.1275 ; 357.0924 ; 343.08$ \\
\hline 289 & 27.14 & {$[\mathrm{M}-\mathrm{H}]^{-}$} & 837.3927 & 837.3903 & 2.834 & $\mathrm{C}_{42} \mathrm{H}_{62} \mathrm{O}_{17}$ & Licorice saponin P2 & $837.4260 ; 351.0647 ; 193.03$ \\
\hline 290 & 27.14 & $\begin{array}{l}{[\mathrm{M}+} \\
\mathrm{FA}-\mathrm{H}]^{-}\end{array}$ & 843.4760 & 843.4737 & 2.712 & $\mathrm{C}_{42} \mathrm{H}_{70} \mathrm{O}_{14}$ & Hydroxysaikosaponin A & $843.4743 ; 797.4672 ; 635.41$ \\
\hline 291 & 27.14 & $\begin{array}{l}{[\mathrm{M}+} \\
\mathrm{H}]^{+}\end{array}$ & 333.1692 & 333.1697 & -1.292 & $\mathrm{C}_{19} \mathrm{H}_{24} \mathrm{O}_{5}$ & Marmin & $333.16861 ; 315.15884$ \\
\hline 292 & 27.20 & {$[\mathrm{M}-\mathrm{H}]^{-}$} & 723.2152 & 723.2131 & 2.848 & $\mathrm{C}_{33} \mathrm{H}_{40} \mathrm{O}_{18}$ & Melitidin & $\begin{array}{l}\text { 723.2244; } 417.1237 ; 402.09 \\
125.0249 ; 99.0462\end{array}$ \\
\hline 293 & 27.21 & $\begin{array}{l}{[\mathrm{M}+} \\
\mathrm{H}]^{+}\end{array}$ & 359.0756 & 359.0761 & -1.459 & $\mathrm{C}_{18} \mathrm{H}_{14} \mathrm{O}_{8}$ & Dichtomitin & $\begin{array}{l}359.0761 ; 326.0421 ; 270.05 \\
256.0364 ; 181.0130\end{array}$ \\
\hline 294 & 27.21 & $\begin{array}{l}{[\mathrm{M}+} \\
\mathrm{H}]^{+}\end{array}$ & 419.1332 & 419.1337 & -1.214 & $\mathrm{C}_{21} \mathrm{H}_{22} \mathrm{O}_{9}$ & Natsudaidain & $419.1327 ; 389.0903$ \\
\hline 295 & 27.44 & $\begin{array}{l}{[\mathrm{M}+} \\
\mathrm{H}]^{+}\end{array}$ & 545.3465 & 545.3473 & -1.431 & $\mathrm{C}_{32} \mathrm{H}_{48} \mathrm{O}_{7}$ & Alisol M 23-acetate & $\begin{array}{l}467.3163 ; 387.2516 ; 299.19 \\
\text { 201.1624; } 145.0996 ; 133.10 \\
\text { 119.0860; }\end{array}$ \\
\hline 296 & 27.44 & {$[\mathrm{M}-\mathrm{H}]-$} & 895.3979 & 895.3958 & 2.328 & $\mathrm{C}_{44} \mathrm{H}_{64} \mathrm{O}_{19}$ & Uralsaponin F & 351.0572 \\
\hline 297 & 27.52 & $\begin{array}{l}{[\mathrm{M}+} \\
\mathrm{H}]^{+}\end{array}$ & 221.1898 & 221.1900 & -1.003 & $\mathrm{C}_{15} \mathrm{H}_{24} \mathrm{O}$ & 10a-Hydroxy-1aH,5ßH-guaia-3,6-diene & $\begin{array}{l}\text { 105.0695; } 91.0538 ; 79.0573 \\
67.0542 ; 119.0842\end{array}$ \\
\hline 298 & 27.53 & {$[\mathrm{M}-\mathrm{H}]^{-}$} & 313.0720 & 313.0707 & 4.234 & $\mathrm{C}_{17} \mathrm{H}_{14} \mathrm{O}_{6}$ & 3',4'-Dihydroxy-7,5'-dime thoxyflavone & $\begin{array}{l}313.0734 ; 298.0487 ; 283.02 \\
276.8391 ; 164.9813 ; 117.03\end{array}$ \\
\hline 299 & 27.53 & $\begin{array}{l}{[\mathrm{M}+} \\
\mathrm{FA}-\mathrm{H}]^{-}\end{array}$ & 521.3121 & 521.3109 & 2.370 & $\mathrm{C}_{28} \mathrm{H}_{44} \mathrm{O}_{6}$ & Polyporusterone B & $475.3211 ; 313.0729$ \\
\hline $300 *$ & 27.68 & $\begin{array}{l}{[\mathrm{M}+} \\
\mathrm{H}]^{+}\end{array}$ & 373.1277 & 373.1282 & -1.312 & $\mathrm{C}_{20} \mathrm{H}_{20} \mathrm{O}_{7}$ & Sinensetin & $\begin{array}{l}373.1290 ; 358.1057 ; 357.09 \\
343.0827\end{array}$ \\
\hline 301 & 27.70 & $\begin{array}{l}{[\mathrm{M}+} \\
\mathrm{H}]^{+}\end{array}$ & 403.1381 & 403.1387 & -1.622 & $\mathrm{C}_{21} \mathrm{H}_{22} \mathrm{O}_{8}$ & Hexamethoxyfalvone & $\begin{array}{l}403.1427 ; 387.1069 ; 373.09 \\
355.0892 ; 327.0889\end{array}$ \\
\hline $302^{*}$ & 27.74 & $\begin{array}{l}{[\mathrm{M}+} \\
\mathrm{H}]^{+}\end{array}$ & 387.1069 & 387.1074 & -1.483 & $\mathrm{C}_{20} \mathrm{H}_{18} \mathrm{O}_{8}$ & Irisflorentin & $\begin{array}{l}387.1098 ; 372.0863 ; 357.06 \\
326.0799\end{array}$ \\
\hline 303 & 27.76 & {$[\mathrm{M}-\mathrm{H}]^{-}$} & 853.3872 & 853.3852 & 2.307 & $\mathrm{C}_{42} \mathrm{H}_{62} \mathrm{O}_{18}$ & 22-Hydroxy-licorice saponin G2 & $853.3958 ; 351.0544$ \\
\hline 304 & 28.04 & $\begin{array}{l}{[\mathrm{M}+} \\
\text { FA-H]- }\end{array}$ & 523.3278 & 523.3265 & 2.437 & $\mathrm{C}_{28} \mathrm{H}_{46} \mathrm{O}_{6}$ & Polyporusterone A & $477.3126 ; 433.1752 ; 343.14$ \\
\hline
\end{tabular}




\begin{tabular}{|c|c|c|c|c|c|c|c|c|}
\hline No. & $\begin{array}{l}\mathrm{RT} \\
/ \mathrm{min}\end{array}$ & $\begin{array}{l}\text { lon } \\
\text { model }\end{array}$ & $\begin{array}{l}\text { Measured } \\
\text { mass /Da }\end{array}$ & $\begin{array}{l}\text { Calculated } \\
\text { mass / Da }\end{array}$ & $\begin{array}{l}\text { Error } \\
\text { /ppm }\end{array}$ & $\begin{array}{l}\text { Molecular } \\
\text { formula }\end{array}$ & Identification & Fragment ions \\
\hline 305 & 28.09 & {$[\mathrm{M}-\mathrm{H}]^{-}$} & 373.0929 & 373.0918 & 2.965 & $\mathrm{C}_{19} \mathrm{H}_{18} \mathrm{O}_{8}$ & 5,7-Hydroxy-3',4',5',6-tetramethoxyflavone & $\begin{array}{l}373.0934 ; 358.0701 ; 343.04 \\
328.0224\end{array}$ \\
\hline 306 & 28.12 & $\begin{array}{l}{[\mathrm{M}+} \\
\mathrm{H}]^{+}\end{array}$ & 531.3649 & 531.3680 & -5.920 & $\mathrm{C}_{32} \mathrm{H}_{50} \mathrm{O}_{6}$ & Alisol N 23-acetate & $\begin{array}{l}\text { 453.3347; 381.2838; } 229.14 \\
\text { 207.1747; }\end{array}$ \\
\hline 307 & 28.13 & $\begin{array}{l}{[2 \mathrm{M}-} \\
\mathrm{H}]^{-}\end{array}$ & 295.0976 & 295.0965 & 3.641 & $\mathrm{C}_{9} \mathrm{H}_{8} \mathrm{O}_{2}$ & E-cinnamic acid & 295.0971 \\
\hline 308 & 28.15 & {$[\mathrm{M}-\mathrm{H}]^{-}$} & 487.1248 & 487.1235 & 2.735 & $\mathrm{C}_{24} \mathrm{H}_{24} \mathrm{O}_{11}$ & Agastachoside isomer & $487.1285 ; 283.0613 ; 268.03$ \\
\hline 309 & 28.23 & {$[\mathrm{M}-\mathrm{H}]-$} & 285.0769 & 285.0758 & 4.034 & $\mathrm{C}_{16} \mathrm{H}_{14} \mathrm{O}_{5}$ & Homobutein & $123.0457 ; 117.0348 ; 108.02$ \\
\hline 310 & 28.27 & {$[M-H]^{-}$} & 819.3818 & 819.3798 & 2.536 & $\mathrm{C}_{42} \mathrm{H}_{60} \mathrm{O}_{16}$ & Licorice saponin E2 & $819.3898 ; 351.0554$ \\
\hline 311 & 28.36 & $\begin{array}{l}{[\mathrm{M}+} \\
\mathrm{H}]^{+}\end{array}$ & 531.3653 & 531.3680 & -5.111 & $\mathrm{C}_{32} \mathrm{H}_{50} \mathrm{O}_{6}$ & 16ß-Hydroxyalisol B 23-acetate & $213.1665 ; 147.1158$ \\
\hline $312^{*}$ & 28.53 & $\begin{array}{l}{[\mathrm{M}+} \\
\mathrm{H}]^{+}\end{array}$ & 285.0751 & 285.0758 & -2.385 & $\mathrm{C}_{16} \mathrm{H}_{12} \mathrm{O}_{5}$ & Oroxylin A & $\begin{array}{l}285.0764 ; 270.0526 ; 242.05 \\
153.0187\end{array}$ \\
\hline $313^{*}$ & 28.7 & $\begin{array}{l}{[\mathrm{M}+} \\
\mathrm{Na}]^{+}\end{array}$ & 317.1718 & 317.1723 & -1.326 & $\mathrm{C}_{17} \mathrm{H}_{26} \mathrm{O}_{4}$ & 6-Gingerol & $317.1711 ; 217.0801$ \\
\hline 314 & 28.75 & {$[\mathrm{M}-\mathrm{H}]^{-}$} & 879.4030 & 879.4009 & 2.341 & $\mathrm{C}_{44} \mathrm{H}_{64} \mathrm{O}_{18}$ & 22-Acetoxyl-glycyrrhizin & $879.4120 ; 351.0628$ \\
\hline 315 & 29.05 & $\begin{array}{l}{[\mathrm{M}+} \\
\mathrm{H}]^{+}\end{array}$ & 704.3972 & 704.3978 & -0.756 & $\mathrm{C}_{34} \mathrm{H}_{53} \mathrm{~N}_{7} \mathrm{O}_{9}$ & Citrusin I & $686.3947 ; 555.3062$ \\
\hline 316 & 29.06 & {$[\mathrm{M}-\mathrm{H}]^{-}$} & 343.0825 & 343.0812 & 3.675 & $\mathrm{C}_{18} \mathrm{H}_{16} \mathrm{O}_{7}$ & 3',4',5'-Trimethoxyflavone & $343.0837 ; 328.0560 ; 313.04$ \\
\hline 317 & 29.06 & {$[\mathrm{M}-\mathrm{H}]-$} & 863.4076 & 863.4060 & 1.869 & $\mathrm{C}_{44} \mathrm{H}_{64} \mathrm{O}_{17}$ & 22ß-Acetoxyglycyrrhaldehyde & $801.4087 ; 351.0576$ \\
\hline 318 & 29.06 & {$[\mathrm{M}-\mathrm{H}]^{-}$} & 983.4501 & 983.4482 & 1.927 & $\mathrm{C}_{48} \mathrm{H}_{72} \mathrm{O}_{21}$ & Licorice saponin A3 & 983.4489 \\
\hline 319 & 29.08 & {$[\mathrm{M}-\mathrm{H}]-$} & 879.4029 & 879.4009 & 2.273 & $\mathrm{C}_{44} \mathrm{H}_{64} \mathrm{O}_{18}$ & $\begin{array}{l}22 \beta \text {-Acetoxyl-glycyrrhizin or uralsaponin } \\
M\end{array}$ & 351.0578 \\
\hline $320 *$ & 29.30 & $\begin{array}{l}{[\mathrm{M}+} \\
\mathrm{H}]^{+}\end{array}$ & 403.1382 & 403.1387 & -1.474 & $\mathrm{C}_{21} \mathrm{H}_{22} \mathrm{O}_{8}$ & Nobiletin & $\begin{array}{l}403.1385 ; 388.1151 ; 373.09 \\
355.0811 ; 327.0856\end{array}$ \\
\hline 321 & 29.38 & $\begin{array}{l}{[\mathrm{M}+} \\
\mathrm{H}]^{+}\end{array}$ & 505.3517 & 505.3524 & -1.416 & $\mathrm{C}_{30} \mathrm{H}_{48} \mathrm{O}_{6}$ & 16-Oxoalisol A & 505.3501 \\
\hline 322 & 29.42 & $\begin{array}{l}{[\mathrm{M}+} \\
\mathrm{H}]^{+}\end{array}$ & 285.0752 & 285.0758 & -1.929 & $\mathrm{C}_{16} \mathrm{H}_{12} \mathrm{O}_{5}$ & Batatasin I & $\begin{array}{l}285.0752 ; 270.0912 ; 254.09 \\
253.0897 ; 225.0911\end{array}$ \\
\hline 323 & 29.42 & $\begin{array}{l}{[\mathrm{M}+} \\
\mathrm{H}]^{+}\end{array}$ & 547.3623 & 547.3629 & -1.152 & $\mathrm{C}_{32} \mathrm{H}_{50} \mathrm{O}_{7}$ & 16-Oxo-alisol A-23-acetate & $\begin{array}{l}\text { 451.3238; 415.2820; 353.24 } \\
241.1619 ; 149.0962 ;\end{array}$ \\
\hline 324 & 29.43 & {$[\mathrm{M}-\mathrm{H}]^{-}$} & 837.3922 & 837.3903 & 2.177 & $\mathrm{C}_{42} \mathrm{H}_{62} \mathrm{O}_{17}$ & Yunganoside $\mathrm{K} 2$ or Macedonoside A & $837.3912 ; 351.0547$ \\
\hline $325^{*}$ & 29.53 & $\begin{array}{l}{[\mathrm{M}+} \\
\mathrm{H}]^{+}\end{array}$ & 285.0750 & 285.0758 & -2.806 & $\mathrm{C}_{16} \mathrm{H}_{12} \mathrm{O}_{5}$ & Acacetin & $\begin{array}{l}285.0768 ; 270.0506 ; 242.05 \\
153.0191\end{array}$ \\
\hline 326 & 29.53 & {$[\mathrm{M}-\mathrm{H}]^{-}$} & 329.2335 & 329.2323 & 3.673 & $\mathrm{C}_{18} \mathrm{H}_{34} \mathrm{O}_{5}$ & Pinellic acid & $329.2385 ; 229.1465 ; 211.13$ \\
\hline 327 & 29.57 & $\begin{array}{l}{[\mathrm{M}+} \\
\mathrm{H}]^{+}\end{array}$ & 531.3654 & 531.3680 & -4.885 & $\mathrm{C}_{32} \mathrm{H}_{50} \mathrm{O}_{6}$ & Alisol F 24-acetate & 513.1300; 163.1156; \\
\hline 328 & 29.65 & $\begin{array}{l}{[\mathrm{M}+} \\
\mathrm{H}]^{+}\end{array}$ & 489.3570 & 489.3575 & -0.860 & $\mathrm{C}_{30} \mathrm{H}_{48} \mathrm{O}_{5}$ & Alisol F or isomer & $\begin{array}{l}\text { 471.3458; } 453.3369 ; 399.28 \\
219.1701 ;\end{array}$ \\
\hline 329 & 29.66 & $\begin{array}{l}{[\mathrm{M}+} \\
\mathrm{FA}-\mathrm{H}]^{-}\end{array}$ & 971.5229 & 971.5210 & 1.939 & $\mathrm{C}_{48} \mathrm{H}_{78} \mathrm{O}_{17}$ & Saikosaponin C & $971.5364 ; 779.4565 ; 617.44$ \\
\hline 330 & 29.80 & {$[\mathrm{M}-\mathrm{H}]^{-}$} & 329.2336 & 329.2323 & 4.220 & $\mathrm{C}_{18} \mathrm{H}_{34} \mathrm{O}_{5}$ & Pinellic acid isomer & $\begin{array}{l}\text { 293.2103; 229.1433; } 171.10 \\
\text { 139.1133; } 127.1152 ;\end{array}$ \\
\hline 331 & 29.81 & $\begin{array}{l}{[\mathrm{M}+} \\
\mathrm{H}]^{+}\end{array}$ & 433.1488 & 433.1493 & -1.105 & $\mathrm{C}_{22} \mathrm{H}_{24} \mathrm{O}_{9}$ & $3,5,6,7,8^{\prime}, 3^{\prime}, 4^{\prime}$-Heptemthoxyflavone & $\begin{array}{l}\text { 433.1453; } 418.1216 ; 417.11 \\
403.1007 ; 385.0913\end{array}$ \\
\hline $332 *$ & 29.98 & $\begin{array}{l}{[\mathrm{M}+} \\
\mathrm{H}]^{+}\end{array}$ & 231.1377 & 231.1380 & -0.979 & $\mathrm{C}_{15} \mathrm{H}_{18} \mathrm{O}_{2}$ & Atractylenolide- $\mathbb{\Downarrow}$ & $\begin{array}{l}231.1381 ; 213.1315 ; 203.11 \\
185.1321\end{array}$ \\
\hline 333 & 30.18 & $\begin{array}{l}{[\mathrm{M}+} \\
\mathrm{H}]^{+}\end{array}$ & 419.1333 & 419.1337 & -0.927 & $\mathrm{C}_{21} \mathrm{H}_{22} \mathrm{O}_{9}$ & Gardenin A & $\begin{array}{l}\text { 419.1322; 404.1076; } 389.08 \\
371.0741 ; 165.0535\end{array}$ \\
\hline
\end{tabular}




\begin{tabular}{|c|c|c|c|c|c|c|c|c|}
\hline No. & $\begin{array}{l}\mathrm{RT} \\
\text { /min }\end{array}$ & $\begin{array}{l}\text { lon } \\
\text { model }\end{array}$ & $\begin{array}{l}\text { Measured } \\
\text { mass /Da }\end{array}$ & $\begin{array}{l}\text { Calculated } \\
\text { mass /Da }\end{array}$ & $\begin{array}{l}\text { Error } \\
\text { /ppm }\end{array}$ & $\begin{array}{l}\text { Molecular } \\
\text { formula }\end{array}$ & Identification & Fragment ions \\
\hline 334 & 30.19 & {$[\mathrm{M}-\mathrm{H}]^{-}$} & 837.3925 & 837.3903 & 2.548 & $\mathrm{C}_{42} \mathrm{H}_{62} \mathrm{O}_{17}$ & Licorice saponin G2 isomer & $837.3966 ; 351.0610$ \\
\hline 335 & 30.38 & $\begin{array}{l}{[\mathrm{M}+} \\
\mathrm{FA}-\mathrm{H}]^{-}\end{array}$ & 973.5385 & 973.5367 & 1.842 & $\mathrm{C}_{48} \mathrm{H}_{80} \mathrm{O}_{17}$ & Saikosaponin F & $\begin{array}{l}973.5410 ; 927.5316 ; 781.47 \\
619.4630\end{array}$ \\
\hline 336 & 30.45 & $\begin{array}{l}{[\mathrm{M}+} \\
\mathrm{H}]^{+}\end{array}$ & 329.1016 & 329.1020 & -1.139 & $\mathrm{C}_{18} \mathrm{H}_{16} \mathrm{O}_{6}$ & Monohydroxytrimethoxyflavone & $\begin{array}{l}329.1027 ; 314.0788 ; 296.06 \\
268.0744 ; 240.0783\end{array}$ \\
\hline 337 & 30.55 & $\begin{array}{l}{[\mathrm{M}+} \\
\mathrm{H}]^{+}\end{array}$ & 274.2736 & 274.2741 & -1.699 & $\mathrm{C}_{16} \mathrm{H}_{35} \mathrm{NO}_{2}$ & Hexadecyl dihydrosphingosine & $274.2739 ; 256.2890$ \\
\hline $338^{*}$ & 30.76 & $\begin{array}{l}{[\mathrm{M}+} \\
\mathrm{H}]^{+}\end{array}$ & 373.1284 & 373.1282 & -1.580 & $\mathrm{C}_{20} \mathrm{H}_{20} \mathrm{O}_{7}$ & Tangeretin & $373.1929 ; 358.1043 ; 343.08$ \\
\hline 339 & 30.89 & $\begin{array}{l}{[\mathrm{M}+} \\
\mathrm{FA}-\mathrm{H}]^{-}\end{array}$ & 531.3330 & 531.3316 & 2.484 & $\mathrm{C}_{30} \mathrm{H}_{46} \mathrm{O}_{5}$ & 16-0xo-11-anhydroalisol A & $\begin{array}{l}485.3298 ; 467.3206 ; 367.26 \\
245.1579 ;\end{array}$ \\
\hline 340 & 30.91 & {$[\mathrm{M}-\mathrm{H}]^{-}$} & 329.2336 & 329.2323 & 3.947 & $\mathrm{C}_{18} \mathrm{H}_{34} \mathrm{O}_{5}$ & Pinellic acid isomer & $\begin{array}{l}229.1451 ; 211.1352 ; 183.14 \\
171.1041 ; 118.9584 ; 99.082\end{array}$ \\
\hline $341^{*}$ & 30.94 & $\begin{array}{l}{[\mathrm{M}+} \\
\mathrm{Na}]^{+}\end{array}$ & 377.0991 & 377.0994 & -1.165 & $\mathrm{C}_{20} \mathrm{H}_{18} \mathrm{O}_{6}$ & Asarinin & 377.0990 \\
\hline 342 & 30.98 & {$[\mathrm{M}-\mathrm{H}]^{-}$} & 837.3923 & 837.3903 & 2.321 & $\mathrm{C}_{42} \mathrm{H}_{62} \mathrm{O}_{17}$ & Licorice saponin Q2 & $837.3956 ; 351.0559 ; 193.03$ \\
\hline 343 & 31.02 & {$[\mathrm{M}-\mathrm{H}]^{-}$} & 367.1188 & 367.1176 & 3.337 & $\mathrm{C}_{21} \mathrm{H}_{20} \mathrm{O}_{6}$ & Glycycoumarin & $\begin{array}{l}367.1208 ; 309.0429 ; 297.04 \\
284.0338\end{array}$ \\
\hline 344 & 31.2 & $\begin{array}{l}{[\mathrm{M}+} \\
\mathrm{FA}-\mathrm{H}]^{-}\end{array}$ & 533.3488 & 533.3473 & 2.756 & $\mathrm{C}_{30} \mathrm{H}_{48} \mathrm{O}_{5}$ & Alisol F & $487.3427 ; 353.2485 ; 203.11$ \\
\hline 345 & 31.26 & {$[\mathrm{M}-\mathrm{H}]^{-}$} & 353.1399 & 353.1384 & 4.275 & $\mathrm{C}_{21} \mathrm{H}_{22} \mathrm{O}_{5}$ & Gancaonin I & $335.1210 ; 150.0335$ \\
\hline 346 & 31.39 & {$[\mathrm{M}-\mathrm{H}]^{-}$} & 353.1034 & 353.1020 & 4.121 & $\mathrm{C}_{20} \mathrm{H}_{18} \mathrm{O}_{6}$ & Licoisoflavone A & $353.1027 ; 316.8315 ; 269.04$ \\
\hline 347 & 31.40 & $\begin{array}{l}{[\mathrm{M}+} \\
\mathrm{H}]^{+}\end{array}$ & 403.1383 & 403.1387 & -1.002 & $\mathrm{C}_{21} \mathrm{H}_{22} \mathrm{O}_{8}$ & Hexamethoxyfalvone & $\begin{array}{l}403.1393 ; 388.1228 ; 373.09 \\
355.0789 ; 301.0740\end{array}$ \\
\hline 348 & 31.61 & $\begin{array}{l}{[\mathrm{M}+} \\
\mathrm{FA}-\mathrm{H}]^{-}\end{array}$ & 573.3438 & 573.3422 & 2.817 & $\mathrm{C}_{32} \mathrm{H}_{48} \mathrm{O}_{6}$ & Alisol C 23-acetate & $527.3486 ; 509.3313 ; 449.31$ \\
\hline 349 & 31.62 & $\begin{array}{l}{[\mathrm{M}+} \\
\mathrm{H}]^{+}\end{array}$ & 529.3519 & 529.3524 & -0.936 & $\mathrm{C}_{32} \mathrm{H}_{48} \mathrm{O}_{6}$ & 16-0xo-11-anhydroalisol A 24-acetate & $\begin{array}{l}511.3391 ; 469.3290 ; 451.32 \\
433.3083 ; ; 203.1442 ;\end{array}$ \\
\hline 350 & 31.63 & $\begin{array}{l}{[\mathrm{M}+} \\
\mathrm{H}]^{+}\end{array}$ & 487.3429 & 487.3418 & 2.317 & $\mathrm{C}_{30} \mathrm{H}_{46} \mathrm{O}_{5}$ & Alisol C & $\begin{array}{l}\text { 469.3331; 415.2848; } 217.15 \\
161.0951 ; 119.0853 ;\end{array}$ \\
\hline 351 & 31.63 & $\begin{array}{l}{[\mathrm{M}+} \\
\mathrm{FA}-\mathrm{H}]^{-}\end{array}$ & 533.3484 & 533.3473 & 2.531 & $\mathrm{C}_{30} \mathrm{H}_{48} \mathrm{O}_{5}$ & $13 \beta, 17 \beta$-epoxyalisol B & $487.3427 ; 353.2485 ; 203.11$ \\
\hline 352 & 31.63 & {$[\mathrm{M}-\mathrm{H}]^{-}$} & 837.3925 & 837.3903 & 2.548 & $\mathrm{C}_{42} \mathrm{H}_{62} \mathrm{O}_{17}$ & Licorice saponin G2 & $837.4026 ; 351.0586 ; 193.04$ \\
\hline 353 & 31.65 & {$[\mathrm{M}-\mathrm{H}]^{-}$} & 967.4555 & 967.4533 & 2.263 & $\mathrm{C}_{48} \mathrm{H}_{72} \mathrm{O}_{20}$ & Rhaoglycyrrhizin & $967.4653 ; 497.1171$ \\
\hline 354 & 31.85 & $\begin{array}{l}{[\mathrm{M}+} \\
\mathrm{H}]^{+}\end{array}$ & 471.3463 & 471.3469 & -1.287 & $\mathrm{C}_{30} \mathrm{H}_{46} \mathrm{O}_{4}$ & 16,23-Oxido-alisol B & $\begin{array}{l}453.3332 ; 399.2878 ; 425.34 \\
201.1686 ; 177.1286 ; 155.08\end{array}$ \\
\hline $355^{\star}$ & 31.86 & {$[\mathrm{M}-\mathrm{H}]^{-}$} & 821.3973 & 821.3954 & 2.347 & $\mathrm{C}_{42} \mathrm{H}_{62} \mathrm{O}_{16}$ & Glycyrrhizic acid & $\begin{array}{l}821.3973 ; 645.3638 ; 469.33 \\
351.0563 ; 193.0341 ; 175.02\end{array}$ \\
\hline 356 & 31.86 & {$[\mathrm{M}-\mathrm{H}]^{-}$} & 823.4034 & 823.4111 & -9.330 & $\mathrm{C}_{42} \mathrm{H}_{64} \mathrm{O}_{16}$ & Uralsaponin C & $823.4174 ; 351.0595$ \\
\hline 357 & 31.89 & $\begin{array}{l}{[\mathrm{M}+} \\
\mathrm{H}]^{+}\end{array}$ & 531.3658 & 531.3680 & -4.208 & $\mathrm{C}_{32} \mathrm{H}_{50} \mathrm{O}_{6}$ & Alisol F 24-acetate isomer & $\begin{array}{l}\text { 495.3526; } 453.3417 ; 276.94 \\
217.1596 ; 173.1322 ; 145.10 \\
121.1027 ;\end{array}$ \\
\hline $358^{*}$ & 31.96 & {$[\mathrm{M}-\mathrm{H}]^{-}$} & 337.1447 & 337.1434 & 3.780 & $\mathrm{C}_{21} \mathrm{H}_{22} \mathrm{O}_{4}$ & Licochalcone A & $\begin{array}{l}266.9918 ; 216.9262 ; 198.91 \\
96.9702 ; 78.9587\end{array}$ \\
\hline 359 & 32.21 & {$[\mathrm{M}-\mathrm{H}]^{-}$} & 351.0876 & 351.0863 & 3.718 & $\mathrm{C}_{20} \mathrm{H}_{16} \mathrm{O}_{6}$ & Licoisoflavone B & $\begin{array}{l}351.0878 ; 336.0748 ; 307.09 \\
283.1001 ; 265.0873 ; 199.07\end{array}$ \\
\hline $360^{*}$ & 32.26 & $\begin{array}{l}{[\mathrm{M}+} \\
\mathrm{Na}]^{+}\end{array}$ & 345.2033 & 345.2034 & -1.016 & $\mathrm{C}_{19} \mathrm{H}_{30} \mathrm{O}_{4}$ & 8-Gingerol & 345.2031 \\
\hline $361^{*}$ & 32.30 & {$[\mathrm{M}-\mathrm{H}]$} & 353.1032 & 353.1032 & 2.612 & $\mathrm{C}_{20} \mathrm{H}_{18} \mathrm{O}_{6}$ & Isolicoflavonol & 353.1022 \\
\hline
\end{tabular}




\begin{tabular}{|c|c|c|c|c|c|c|c|c|}
\hline No. & $\begin{array}{l}\mathrm{RT} \\
/ \mathrm{min}\end{array}$ & $\begin{array}{l}\text { lon } \\
\text { model }\end{array}$ & $\begin{array}{l}\text { Measured } \\
\text { mass /Da }\end{array}$ & $\begin{array}{l}\text { Calculated } \\
\text { mass } / \mathrm{Da}\end{array}$ & $\begin{array}{l}\text { Error } \\
\text { /ppm }\end{array}$ & $\begin{array}{l}\text { Molecular } \\
\text { formula }\end{array}$ & Identification & Fragment ions \\
\hline 362 & 32.35 & {$[\mathrm{M}-\mathrm{H}]^{-}$} & 985.4661 & 985.4639 & 2.207 & $\mathrm{C}_{48} \mathrm{H}_{74} \mathrm{O}_{21}$ & Yunganoside D1 or Yunganoside G1 & $985.4770 ; 497.1194$ \\
\hline 363 & 32.36 & $\begin{array}{l}{[\mathrm{M}+} \\
\mathrm{H}]^{+}\end{array}$ & 529.3497 & 529.3524 & -5.092 & $\mathrm{C}_{32} \mathrm{H}_{48} \mathrm{O}_{6}$ & Alisol Q 23-acetate & 451.3220; \\
\hline 364 & 32.39 & {$[\mathrm{M}-\mathrm{H}]^{-}$} & 807.4185 & 807.4161 & 2.938 & $\mathrm{C}_{42} \mathrm{H}_{64} \mathrm{O}_{15}$ & Licoricesaponin B2 & 807.4230; 351.0564; 193.03 \\
\hline 365 & 32.50 & {$[\mathrm{M}-\mathrm{H}]^{-}$} & 837.3925 & 837.3903 & 2.619 & $\mathrm{C}_{42} \mathrm{H}_{62} \mathrm{O}_{17}$ & Uralsaponin N & 837.3998; 351.0582; 193.03 \\
\hline 366 & 32.66 & {$[\mathrm{M}-\mathrm{H}]^{-}$} & 807.4184 & 807.4161 & 2.790 & $\mathrm{C}_{42} \mathrm{H}_{64} \mathrm{O}_{15}$ & 22-Dehydroxyural saponin & $807.4214 ; 351.0548 ; 193.03$ \\
\hline 367 & 32.80 & {$[\mathrm{M}-\mathrm{H}]^{-}$} & 821.3976 & 821.3954 & 2.712 & $\mathrm{C}_{42} \mathrm{H}_{62} \mathrm{O}_{16}$ & Licorice saponin $\mathrm{H} 2$ & $821.4034 ; 351.0593 ; 193.03$ \\
\hline 368 & 32.81 & $\begin{array}{l}{[\mathrm{M}+} \\
\mathrm{H}- \\
\left.\mathrm{H}_{2} \mathrm{O}\right]^{+}\end{array}$ & 471.3464 & 471.3469 & -1.096 & $\mathrm{C}_{30} \mathrm{H}_{48} \mathrm{O}_{5}$ & 13B,17B-Epoxyalisol B & $\begin{array}{l}\text { 381.2803; } 310.2266 ; 159.11 \\
\text { 133.1009; }\end{array}$ \\
\hline 369 & 32.99 & $\begin{array}{l}{[\mathrm{M}+} \\
\mathrm{FA}-\mathrm{H}]^{-}\end{array}$ & 867.4747 & 867.4737 & 1.231 & $\mathrm{C}_{44} \mathrm{H}_{70} \mathrm{O}_{14}$ & 3"-O-Acetylsaikosaponin A & $\begin{array}{l}867.4879 ; 821.4796 ; 779.46 \\
761.4559 ; 617.4142\end{array}$ \\
\hline 370 & 33.12 & $\begin{array}{l}{[\mathrm{M}+} \\
\mathrm{H}]^{+}\end{array}$ & 248.2005 & 248.2009 & -1.575 & $\mathrm{C}_{16} \mathrm{H}_{25} \mathrm{NO}$ & $\mathrm{N}$-Isobutyl-dodecatetraenamide & $\begin{array}{l}267.1306 ; 152.1072 ; 107.08 \\
96.0442 ; 79.0538\end{array}$ \\
\hline 371 & 33.16 & $\begin{array}{l}{[\mathrm{M}+} \\
\mathrm{H}]^{+}\end{array}$ & 531.3665 & 531.3680 & -2.815 & $\mathrm{C}_{32} \mathrm{H}_{50} \mathrm{O}_{6}$ & Alisol D & $435.3309 ; 337.2465 ; 163.10$ \\
\hline $372^{*}$ & 33.30 & {$[\mathrm{M}-\mathrm{H}]^{-}$} & 353.1032 & 353.1020 & 3.612 & $\mathrm{C}_{20} \mathrm{H}_{18} \mathrm{O}_{6}$ & Licoflavonol & $\begin{array}{l}353.1076 ; 297.0401 ; 214.87 \\
151.0039\end{array}$ \\
\hline 373 & 33.41 & $\begin{array}{l}{[2 \mathrm{M}+} \\
\mathrm{H}]^{+}\end{array}$ & 437.3407 & 437.3414 & -1.548 & $\mathrm{C}_{15} \mathrm{H}_{22} \mathrm{O}$ & Nootkatone or its isomers & $\begin{array}{l}437.3442 ; 346.2790 ; 274.27 \\
265.1953 ; 187.1491 ; 105.06\end{array}$ \\
\hline 374 & 33.42 & $\begin{array}{l}{[\mathrm{M}+} \\
\mathrm{FA}-\mathrm{H}]^{-}\end{array}$ & 779.4589 & 779.4576 & 2.951 & $\mathrm{C}_{42} \mathrm{H}_{68} \mathrm{O}_{13}$ & Saikosaponin A & $\begin{array}{l}\text { 779.4589; } 617.4060 ; 471.34 \\
161.0446 ; 145.0495 ; 113.04 \\
101.0229\end{array}$ \\
\hline 375 & 33.42 & {$[\mathrm{M}-\mathrm{H}]^{-}$} & 865.4600 & 865.4580 & 2.251 & $\mathrm{C}_{45} \mathrm{H}_{70} \mathrm{O}_{16}$ & Malonysaikosaponin A & $\begin{array}{l}865.4662 ; 821.4754 ; 779.46 \\
761.4580 ; 617.4110\end{array}$ \\
\hline 376 & 33.56 & {$[\mathrm{M}-\mathrm{H}]^{-}$} & 821.3977 & 821.3954 & 2.798 & $\mathrm{C}_{42} \mathrm{H}_{62} \mathrm{O}_{16}$ & Licroice Saponin K2 & $821.4061 ; 351.0591 ; 193.03$ \\
\hline 377 & 33.60 & $\begin{array}{l}{[\mathrm{M}+} \\
\mathrm{H}]^{+}\end{array}$ & 487.3433 & 487.3418 & 3.117 & $\mathrm{C}_{30} \mathrm{H}_{46} \mathrm{O}_{5}$ & $\begin{array}{l}(8 a, 9 \beta, 11 \beta, 14 \beta, 16 \beta, 23 S, 24 R)-16,23: 24,25- \\
\text { Diepoxy-11,20-dihydroxy-dammar-13(17)- } \\
\text { en-3-one }\end{array}$ & $\begin{array}{l}\text { 469.3302; 451.3198; } 397.27 \\
353.2457 ; 253.1536 ; 201.12\end{array}$ \\
\hline 378 & 33.60 & $\begin{array}{l}{[\mathrm{M}+} \\
\mathrm{FA}-\mathrm{H}]^{-}\end{array}$ & 867.4758 & 867.4737 & 2.499 & $\mathrm{C}_{44} \mathrm{H}_{70} \mathrm{O}_{14}$ & 4"-O-Acetylsaikosaponin A & $\begin{array}{l}867.4834 ; 821.4784 ; 779.46 \\
761.4489 ; 617.1039\end{array}$ \\
\hline 379 & 34.87 & {$[\mathrm{M}-\mathrm{H}]^{-}$} & 865.4600 & 865.4580 & 2.320 & $\mathrm{C}_{45} \mathrm{H}_{70} \mathrm{O}_{16}$ & Malonysaikosaponin D & $\begin{array}{l}865.4725 ; 821.4783 ; 779.45 \\
761.4546 ; 617.4083\end{array}$ \\
\hline 380 & 34.90 & $\begin{array}{l}{[\mathrm{M}+} \\
\mathrm{H}]^{+}\end{array}$ & 529.3503 & 529.3524 & -3.940 & $\mathrm{C}_{32} \mathrm{H}_{48} \mathrm{O}_{6}$ & Alisol C & $\begin{array}{l}\text { 511.3422; } 451.3223 ; 397.27 \\
\text { 355.2664; } 205.1599 ; 187.14 \\
\text { 145.1003; }\end{array}$ \\
\hline 381 & 34.93 & $\begin{array}{l}{[\mathrm{M}+} \\
\mathrm{FA}-\mathrm{H}]^{-}\end{array}$ & 825.4655 & 825.4631 & 2.935 & $\mathrm{C}_{42} \mathrm{H}_{68} \mathrm{O}_{13}$ & Saikosaponin G & $825.4774 ; 779.4692 ; 617.41$ \\
\hline 382 & 34.94 & $\begin{array}{l}{[2 \mathrm{M}+} \\
\mathrm{H}]+\end{array}$ & 437.3410 & 437.3414 & -0.862 & $\mathrm{C}_{15} \mathrm{H}_{22} \mathrm{O}$ & nootkatone or its isomers & $437.3464 ; 201.1661 ; 163.14$ \\
\hline 383 & 35.05 & $\begin{array}{l}{[\mathrm{M}+} \\
\mathrm{FA}-\mathrm{H}]^{-}\end{array}$ & 867.4759 & 867.4737 & 2.568 & $\mathrm{C}_{44} \mathrm{H}_{70} \mathrm{O}_{14}$ & 3"-O-Acetylsaikosaponin D & $\begin{array}{l}\text { 867.4855; } 821.4813 ; 779.46 \\
761.4565 ; 617.4124\end{array}$ \\
\hline 384 & 35.15 & $\begin{array}{l}{[\mathrm{M}+} \\
\mathrm{FA}-\mathrm{H}]^{-}\end{array}$ & 809.4707 & 809.4682 & 3.109 & $\mathrm{C}_{42} \mathrm{H}_{68} \mathrm{O}_{12}$ & Saikosaponin M & $\begin{array}{l}\text { 809.4758; 763.4699; } 601.41 \\
161.0452\end{array}$ \\
\hline 385 & 35.26 & {$[\mathrm{M}-\mathrm{H}]^{-}$} & 823.4132 & 823.4111 & 2.609 & $\mathrm{C}_{42} \mathrm{H}_{64} \mathrm{O}_{16}$ & Licorice Saponin J2 & $823.4224 ; 351.0595 ; 193.03$ \\
\hline 386 & 35.26 & $\begin{array}{l}{[\mathrm{M}+} \\
\mathrm{FA}-\mathrm{H}]^{-}\end{array}$ & 867.4758 & 867.4737 & 2.430 & $\mathrm{C}_{44} \mathrm{H}_{70} \mathrm{O}_{14}$ & 6"-O-Acetylsaikosaponin A & $\begin{array}{l}867.4855 ; 821.4813 ; 779.46 \\
761.4565 ; 617.4124\end{array}$ \\
\hline 387 & 35.68 & $\begin{array}{l}{[\mathrm{M}+} \\
\mathrm{H}]^{+}\end{array}$ & 219.1741 & 219.1743 & -1.149 & $\mathrm{C}_{15} \mathrm{H}_{22} \mathrm{O}$ & Isolongifolen-9-one & $105.07 ; 91.05 ; 77.04 ; 65.04$ \\
\hline 388 & 35.81 & $\begin{array}{l}{[\mathrm{M}+} \\
\mathrm{FA}-\mathrm{H}]^{-}\end{array}$ & 825.4655 & 825.4631 & 2.935 & $\mathrm{C}_{42} \mathrm{H}_{68} \mathrm{O}_{13}$ & Saikosaponin B2 & $825.4748 ; 779.4655 ; 617.40$ \\
\hline
\end{tabular}




\begin{tabular}{|c|c|c|c|c|c|c|c|c|}
\hline No. & $\begin{array}{l}\mathrm{RT} \\
/ \mathrm{min}\end{array}$ & $\begin{array}{l}\text { lon } \\
\text { model }\end{array}$ & $\begin{array}{l}\text { Measured } \\
\text { mass /Da }\end{array}$ & $\begin{array}{l}\text { Calculated } \\
\text { mass } / \mathrm{Da}\end{array}$ & $\begin{array}{l}\text { Error } \\
\text { /ppm }\end{array}$ & $\begin{array}{l}\text { Molecular } \\
\text { formula }\end{array}$ & Identification & Fragment ions \\
\hline 389 & 36.31 & $\begin{array}{l}{[\mathrm{M}+} \\
\mathrm{H}]^{+}\end{array}$ & 219.1741 & 219.1743 & -1.104 & $\mathrm{C}_{15} \mathrm{H}_{22} \mathrm{O}$ & (Z)-Nuciferol & 219.1728 \\
\hline $390 *$ & 36.43 & $\begin{array}{l}{[\mathrm{M}+} \\
\mathrm{Na}]^{+}\end{array}$ & 413.2296 & 413.2298 & -0.715 & $\mathrm{C}_{23} \mathrm{H}_{34} \mathrm{O}_{5}$ & Tussilagone & 413.2287 \\
\hline $391^{\star}$ & 37.18 & $\begin{array}{l}{[\mathrm{M}+} \\
\mathrm{Na}]^{+}\end{array}$ & 373.2349 & 373.2348 & -0.189 & $\mathrm{C}_{21} \mathrm{H}_{34} \mathrm{O}_{4}$ & 10-Gingerol & $373.2347 ; 217.0815$ \\
\hline 392 & 37.30 & $\begin{array}{l}{[\mathrm{M}+} \\
\mathrm{FA}-\mathrm{H}]^{-}\end{array}$ & 867.4761 & 867.4737 & 2.856 & $\mathrm{C}_{44} \mathrm{H}_{70} \mathrm{O}_{14}$ & 2"-O-Acetylsaikosaponin D & $\begin{array}{l}867.4847 ; 821.4798 ; 779.46 \\
761.4625 ; 617.4105\end{array}$ \\
\hline 393 & 37.55 & $\begin{array}{l}{[\mathrm{M}+} \\
\mathrm{H}]^{+}\end{array}$ & 515.3728 & 515.3731 & -0.584 & $\mathrm{C}_{32} \mathrm{H}_{50} \mathrm{O}_{5}$ & 25-Anhydroalisol A 11-acetate & $\begin{array}{l}\text { 455.3519; } 437.3452 ; 383.29 \\
\text { 339.2678; } 219.1736 ; 159.11 \\
\text { 121.1009; }\end{array}$ \\
\hline 394 & 37.60 & $\begin{array}{l}{[\mathrm{M}+} \\
\mathrm{FA}-\mathrm{H}]^{-}\end{array}$ & 825.4655 & 825.4631 & 2.850 & $\mathrm{C}_{42} \mathrm{H}_{68} \mathrm{O}_{13}$ & Saikosaponin D & $825.4671 ; 779.4666 ; 617.41$ \\
\hline 395 & 38.23 & $\begin{array}{l}{[\mathrm{M}+} \\
\mathrm{H}]^{+}\end{array}$ & 513.3547 & 513.3575 & -5.398 & $\mathrm{C}_{32} \mathrm{H}_{48} \mathrm{O}_{5}$ & Alisol 0 & $\begin{array}{l}\text { 453.3349; 435.3240; } 399.28 \\
\text { 177.1263; 163.1105; }\end{array}$ \\
\hline $396^{*}$ & 38.24 & $\begin{array}{l}{[\mathrm{M}+} \\
\mathrm{FA}-\mathrm{H}]^{-}\end{array}$ & 535.3643 & 535.3629 & 2.596 & $\mathrm{C}_{30} \mathrm{H}_{50} \mathrm{O}_{5}$ & Alisol A & $\begin{array}{l}475.3159 ; 415.0352 ; 329.17 \\
299.0619 ; 269.1501 ; 167.05\end{array}$ \\
\hline 397 & 38.31 & $\begin{array}{l}{[\mathrm{M}+} \\
\mathrm{H}]^{+}\end{array}$ & 529.3495 & 529.3524 & -5.432 & $\mathrm{C}_{32} \mathrm{H}_{48} \mathrm{O}_{6}$ & Alismaketone-C 23-acetate & $\begin{array}{l}\text { 433.3067; } 379.2671 ; 233.15 \\
\text { 145.1009; }\end{array}$ \\
\hline 398 & 38.59 & $\begin{array}{l}{[\mathrm{M}+} \\
\mathrm{FA}-\mathrm{H}]^{-}\end{array}$ & 867.4762 & 867.4737 & 2.925 & $\mathrm{C}_{44} \mathrm{H}_{70} \mathrm{O}_{14}$ & 4"-O-Acetylsaikosaponin D & $\begin{array}{l}867.4873 ; 821.4802 ; 779.46 \\
761.4575 ; 617.4072\end{array}$ \\
\hline 399 & 38.70 & $\begin{array}{l}{[\mathrm{M}+} \\
\mathrm{Na}]^{+}\end{array}$ & 555.3651 & 555.3656 & -0.901 & $\mathrm{C}_{32} \mathrm{H}_{52} \mathrm{O}_{6}$ & Alisol A 24-acetate & 555.3658 \\
\hline 400 & 38.72 & $\begin{array}{l}{[\mathrm{M}+} \\
\mathrm{H}- \\
\left.\mathrm{H}_{2} \mathrm{O}\right]^{+}\end{array}$ & 515.3729 & 515.3731 & -0.351 & $\mathrm{C}_{32} \mathrm{H}_{52} \mathrm{O}_{6}$ & Alisol A 23-acetate & $\begin{array}{l}\text { 497.3585; 437.3370; } 383.29 \\
\text { 365.2855; 339.2698; }\end{array}$ \\
\hline 401 & 38.78 & $\begin{array}{l}{[\mathrm{M}+} \\
\mathrm{H}]^{+}\end{array}$ & 511.3391 & 511.3418 & -5.380 & $\mathrm{C}_{32} \mathrm{H}_{46} \mathrm{O}_{5}$ & Alisol L 23-acetate & $\begin{array}{l}\text { 493.3340; } 451.3210 ; 433.31 \\
\text { 397.2771; 353.2435; } 283.19 \\
\text { 171.1182; }\end{array}$ \\
\hline $402^{*}$ & 39.29 & $\begin{array}{l}{[\mathrm{M}+} \\
\mathrm{FA}-\mathrm{H}]^{-}\end{array}$ & 559.3644 & 559.3629 & 2.592 & $\mathrm{C}_{32} \mathrm{H}_{50} \mathrm{O}_{5}$ & Alisol B-23-acetate & $537.3542 ; 477.3315$ \\
\hline 403 & 39.30 & $\begin{array}{l}{[\mathrm{M}+} \\
\mathrm{H}]^{+}\end{array}$ & 515.3727 & 515.3731 & -0.817 & $\mathrm{C}_{32} \mathrm{H}_{50} \mathrm{O}_{5}$ & 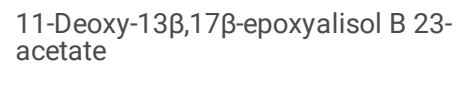 & $\begin{array}{l}383.2960 ; 339.2673 ; 341.28 \\
\text { 203.1418; } 121.1004 ;\end{array}$ \\
\hline 404 & 39.37 & $\begin{array}{l}{[\mathrm{M}+} \\
\mathrm{FA}-\mathrm{H}]^{-}\end{array}$ & 517.3536 & 517.3524 & 2.463 & $\mathrm{C}_{30} \mathrm{H}_{48} \mathrm{O}_{4}$ & Alisol B & 517.3535 \\
\hline $405^{\star}$ & 40.04 & $\begin{array}{l}{[\mathrm{M}+} \\
\mathrm{H}]^{+}\end{array}$ & 529.3873 & 529.3886 & -2.798 & $\mathrm{C}_{33} \mathrm{H}_{52} \mathrm{O}_{5}$ & Pachymic acid & $\begin{array}{l}\text { 493.36; 451.36; } 433.35 ; 355 \\
295.24\end{array}$ \\
\hline
\end{tabular}

* means that the ingredient was confirmed by the reference substance.

RT-retention time; MH means Herba Ephedrae (Mahuang), GC means Radix Glycyrrhizae Praeparata (Zhigancao), XR means Semen Armeniaceae Amarum (Xingren), Ramulus Cinnamoni (Guizhi), ZX means Rhizoma Alismatis (Zixie), BZ means Rhizoma Atractylodis Macrocephalae (Baizhu), FL means Poria (Fuling), CH means Radix Bupleuri (Chaihu), HQ means Radix Scutellariae (Huangqin), BX means Rhizoma Pinelliae prepared with ginger juice (Jiangbanxia), SJ means Rhizoma Zingiberis Recens (Shengjiang), ZW means Radix Asteris (Ziwan), KDH means Flos Farfarae (Kuandonghua), SG means Rhizoma Belamcandae(Shegan), XX means Herba Asari (Xixin), SY means Rhizoma Dioscoreae (Shanyao), ZS means Fructus Aurantii Immaturus (Zhishi), CP means Pericarpium Citri Reticulatae (Chenpi) $30 \mathrm{~g}$ and GHX means Herba Agastachis (Huoxiang)

It is well-accepted that deep understanding of the fragmentation pattern of each authentic standard or its analgous is very helpful for the identification of herbal constituents by using HRMS [17]. In this study, the fragmentation pathways of various constituents (ephedrine, amygdalin, nobiletin, liquiritin, gallic acid, chlorogenic acid, saikosaponin A and glycyrrhizic acid) in QFPDD were carefully studied and proposed in Fig. 2 and Fig. 3. For example, ephedrine ( $t_{R}=$ $7.30 \mathrm{~min}$ ), an abundant alkaloid distributed in Herba Ephedrae, could be easily detected in the positive ion mode with a quasi-molecular ion $[\mathrm{M}+\mathrm{H}]^{+}$at $m / z$ $166.1226\left(\mathrm{C}_{16} \mathrm{H}_{16} \mathrm{NO}\right)$. MS/MS fragmentation analysis demonstrated that this natural compound possessed a neutral loss of $\mathrm{H}_{2} \mathrm{O}$, subsequently by the cleavage of $\mathrm{C}-\mathrm{N}$ bond moiety (Fig. 2A), evidenced by the product ions of $m / z 148.1119\left[\mathrm{M}+\mathrm{H}_{-} \mathrm{H}_{2} \mathrm{O}\right]^{+}, m / z 133.0886\left[\mathrm{M}+\mathrm{H}-\mathrm{H}_{2} \mathrm{O}-\mathrm{CH}\right]^{+}, m / z 117.0701[\mathrm{M}+\mathrm{H}-$ $\left.\mathrm{H}_{2} \mathrm{O}-\mathrm{CH}_{5} \mathrm{~N}\right]^{+}$and $\mathrm{m} / z 91.0548\left[\mathrm{M}+\mathrm{H}-\mathrm{H}_{2} \mathrm{O}-\mathrm{CH}_{5} \mathrm{~N}-\mathrm{C}_{2} \mathrm{H}_{2}\right]^{+}$. The compound $\mathbf{5 6}\left(\mathrm{t}_{\mathrm{R}}=7.30 \mathrm{~min}\right.$ ) in Table 1 were detected in the positive ion mode at the same retention time with the identical $m / z$ of $166.1225\left(\mathrm{C}_{16} \mathrm{H}_{16} \mathrm{NO}\right)$, thus was characterized as ephedrine. amygdalin ( $\left.\mathrm{t}_{\mathrm{R}}=13.93 \mathrm{~min}\right)$, an abundant alkaloid distributed in Semen Armeniaceae Amarum, could be easily detected in the negative ion mode with a quasi-molecular ion $[\mathrm{M}+\mathrm{HCOOH}-\mathrm{H}]^{-}$at $m / z 502.1567$ 
$\left(\mathrm{C}_{21} \mathrm{H}_{28} \mathrm{NO}_{13}\right)$. It was inclined to lose the whole sugar moiety to generate base peak ions $\mathrm{m} / z$ of $323.0972\left[\mathrm{M}+\mathrm{HCOOH}-\mathrm{H}-\mathrm{C}_{6} \mathrm{H}_{11} \mathrm{O}_{6}\right]^{-}$and $179.0550\left[\mathrm{C}_{6} \mathrm{H}_{11} \mathrm{O}_{6}\right]^{-}$ in $\mathrm{MS}^{2}$ spectra (Fig. 2B). Subsequently by cleavage of the functional group on the second glucose, such as $m / z$ of $263.0772\left[\mathrm{M}+\mathrm{HCOOH}-\mathrm{H}-\mathrm{C}_{6} \mathrm{H}_{11} \mathrm{O}_{6}-\mathrm{C}_{2} \mathrm{H}_{4} \mathrm{O}_{2}\right]^{-}$ and $221.0661\left[\mathrm{M}+\mathrm{HCOOH}-\mathrm{H}-\mathrm{C}_{6} \mathrm{H}_{11} \mathrm{O}_{6}-\mathrm{C}_{7} \mathrm{H}_{4} \mathrm{~N}\right]^{-}$. Simultaneously the fragmentation signal of glucose was detected, its $\mathrm{MS} / \mathrm{MS}$ data were shown as $m / z$ of $161.0444\left[\mathrm{C}_{6} \mathrm{H}_{11} \mathrm{O}_{6}-\mathrm{H}_{2} \mathrm{O}\right]^{-}, 119.0335\left[\mathrm{C}_{6} \mathrm{H}_{11} \mathrm{O}_{6}-\mathrm{H}_{2} \mathrm{O}-\mathrm{C}_{2} \mathrm{H}_{2} \mathrm{O}\right]^{-}, 101.0229\left[\mathrm{C}_{6} \mathrm{H}_{11} \mathrm{O}_{6}-\mathrm{H}_{2} \mathrm{O}-\mathrm{C}_{2} \mathrm{H}_{2} \mathrm{O}-\mathrm{H}_{2} \mathrm{O}\right]^{-}$and $89.0229\left[\mathrm{C}_{6} \mathrm{H}_{11} \mathrm{O}_{6}-\mathrm{H}_{2} \mathrm{O}-\mathrm{C}_{2} \mathrm{H}_{2} \mathrm{O}-\mathrm{CH}_{2} \mathrm{O}\right]^{-}$. The compound 90 in Table 1 were detected in the negative ion mode at $13.93 \mathrm{~min}$ with the identical $\mathrm{m} / z$ of $502.1568\left(\mathrm{C}_{21} \mathrm{H}_{28} \mathrm{NO}_{13}\right)$, thus this peak was characterized as amygdalin. Similar to the identification process above, a total of 40 alkaloids were identified from QFPDD, including pseudoephedrine, methylephedrine, ligustrazine, prunasin, et al.

Nobiletin $\left(t_{R}=29.30 \mathrm{~min}\right)$, a flavonoid distributed in Fructus Aurantii Immaturus and Pericarpium Citri Reticulatae, could be easily detected in the positive ion mode with a quasi-molecular ion $[\mathrm{M}+\mathrm{H}]^{+}$at $m / z 403.1385\left(\mathrm{C}_{21} \mathrm{H}_{23} \mathrm{O}_{8}\right)$. MS/MS fragmentation analysis demonstrated that this natural compound possessed demethylation, dehydration, and $\mathrm{CO}$, evidenced by the product ions of $\mathrm{m} / z 388.1151\left[\mathrm{M}+\mathrm{H}-\mathrm{CH}_{3}\right]^{+}, 373.0916\left[\mathrm{M}+\mathrm{H}-\mathrm{CH}_{3}-\mathrm{CH}_{3}\right]+, 355.0811\left[\mathrm{M}+\mathrm{H}-2 \mathrm{CH}_{3}-\mathrm{H}_{2} \mathrm{O}\right]^{+}$ and $327.0856\left[\mathrm{M}+\mathrm{H}-2 \mathrm{CH}_{3}-\mathrm{H}_{2} \mathrm{O}-\mathrm{CO}\right]^{+}$(Fig. 2C). The compound 321 listed in Table 1 were detected in the positive ion mode at $29.30 \mathrm{~min}$ with the identical $\mathrm{m} / \mathrm{z}$ of $403.1382\left(\mathrm{C}_{21} \mathrm{H}_{23} \mathrm{O}_{8}\right)$, thus this peak was characterized as nobiletin. Liquiritin ( $\left.\mathrm{t}_{\mathrm{R}}=18.59 \mathrm{~min}\right)$, a flavonoid glycoside distributed in Radix Glycyrrhizae Praeparata, which was detected in the negative ion mode with a quasi-molecular ion $[\mathrm{M}-\mathrm{H}]^{-}$at $\mathrm{m} / z$ of $417.1195\left(\mathrm{C}_{21} \mathrm{H}_{21} \mathrm{O}_{9}\right)$. Its $\mathrm{MS} / \mathrm{MS}$ data were shown as $\mathrm{m} / z$ of $255.0654\left[\mathrm{M}-\mathrm{H}-\mathrm{C}_{6} \mathrm{H}_{10} \mathrm{O}_{5}\right]^{-}, 153.0178\left[\mathrm{M}-\mathrm{H}-\mathrm{C}_{6} \mathrm{H}_{10} \mathrm{O}_{5}-\mathrm{C}_{8} \mathrm{H}_{6}\right]^{-}, 135.0071\left[\mathrm{M}-\mathrm{H}-\mathrm{C}_{6} \mathrm{H}_{10} \mathrm{O}_{5}-\mathrm{C}_{8} \mathrm{H}_{8} \mathrm{O}\right]^{-}$and $119.0484\left[\mathrm{M}-\mathrm{H}-\mathrm{C}_{6} \mathrm{H}_{10} \mathrm{O}_{5}-\mathrm{C}_{7} \mathrm{H}_{4} \mathrm{O}_{3}\right]^{-}(\mathrm{Fig} .2 \mathrm{D})$. The compound 135 listed in Table 1 could be easily detected in the negative ion mode with the same Rt (at $18.59 \mathrm{~min}$ ) and the identical $\mathrm{m} / z$ of 417.1195 $\left(\mathrm{C}_{21} \mathrm{H}_{21} \mathrm{O}_{9}\right)$, this peak was then characterized as liquiritin. Similar to the identification process above, a total of 162 flavonoids were identified from QFPDD, including isoliquiritin, neoliquiritin, kaempferol, luteolin, quercetin, rutin, hesperidin, neohesperidin, formononetin, isoflavin, formononetin et al.

Gallic acid $\left(\mathrm{t}_{\mathrm{R}}=2.09 \mathrm{~min}\right)$, an organic acid distributed in a variety of herbs, which was detected in the negative ion mode with a quasi-molecular ion [M- $\left.\mathrm{H}\right]^{-}$at the $\mathrm{m} / \mathrm{z}$ of $169.0127\left(\mathrm{C}_{7} \mathrm{H}_{5} \mathrm{O}_{5}\right)$. MS/MS fragmentation analysis demonstrated that this natural compound possessed neutral loss of $\mathrm{H}_{2} \mathrm{O}$, $\mathrm{CO}_{2}$ and/or $\mathrm{CO}$ (Fig. 3A), evidenced by the product ions of $\mathrm{m} / z 151.0020\left[\mathrm{M}-\mathrm{H}-\mathrm{H}_{2} \mathrm{O}\right]^{-}, 125.0226\left[\mathrm{M}-\mathrm{H}-\mathrm{CO}_{2}\right]^{-}, 97.0277\left[\mathrm{M}-\mathrm{H}-\mathrm{CO}_{2}-\mathrm{CO}^{-}, 81.0327\left[\mathrm{M}-\mathrm{H}-2 \mathrm{CO}_{2}\right]^{-}\right.$and $69.0327[\mathrm{M}-\mathrm{H}-$ $\left.\mathrm{CO}_{2}-2 \mathrm{CO}\right]^{-}$. Compound $\mathbf{4 0}$ listed in Table 1 could be detected in the negative ion mode with the same Rt (at $2.09 \mathrm{~min}$ ) and the identical $\mathrm{m} / z$ of 169.0133 $\left(\mathrm{C}_{7} \mathrm{H}_{5} \mathrm{O}_{5}\right)$, this compound was then characterized as gallic acid. Chlorogenic acid ( $\left.t_{R}=12.51 \mathrm{~min}\right)$, an organic acid which was produced from caffeic acid and quinic acid, it was distributed in variety of herbs. It could be easily detected in the positive ion mode with a quasi-molecular ion [M-H] ${ }^{-}$at $m / z 353.0883$ $\left(\mathrm{C}_{16} \mathrm{H}_{17} \mathrm{O}_{9}\right)$. MS/MS fragmentation analysis demonstrated that it was easily broken down into caffeic acid and quinic acid to generate base peak ions $m / z$ of $191.0549\left[\mathrm{M}-\mathrm{H}-\mathrm{C}_{9} \mathrm{H}_{6} \mathrm{O}_{3}\right]^{-}$and $179.0337\left[\mathrm{M}-\mathrm{H}-\mathrm{C}_{7} \mathrm{H}_{10} \mathrm{O}_{5}\right]^{-}$in $\mathrm{MS}^{2}$ spectra (Fig. 3B). Subsequently by neutral loss of $\mathrm{H}_{2} \mathrm{O}$ or $\mathrm{CO}_{2}$, evidenced by the product ions of $\mathrm{m} / \mathrm{z} 173.0442\left[\mathrm{M}-\mathrm{H}-\mathrm{C}_{9} \mathrm{H}_{6} \mathrm{O}_{3}-\mathrm{H}_{2} \mathrm{O}\right]^{-}, 161.0228\left[\mathrm{M}-\mathrm{H}-\mathrm{C}_{7} \mathrm{H}_{10} \mathrm{O}_{5}-\mathrm{H}_{2} \mathrm{O}\right]^{-}, 155.0335\left[\mathrm{M}-\mathrm{H}-\mathrm{C}_{9} \mathrm{H}_{6} \mathrm{O}_{3}-2 \mathrm{H}_{2} \mathrm{O}\right]^{-}$and $135.0435\left[\mathrm{M}-\mathrm{H}_{-} \mathrm{C}_{7} \mathrm{H}_{10} \mathrm{O}_{5}-\mathrm{CO}_{2}\right]^{-}$. Compound 81 listed in Table 1 could be easily detected in the negative ion mode with the same Rt (at $12.51 \mathrm{~min}$ ) and the identical $\mathrm{m} / z$ of $353.0880\left(\mathrm{C}_{16} \mathrm{H}_{17} \mathrm{O}_{9}\right)$, thus this compoundwas characterized as chlorogenic acid. Similar to the identification process above, a total of 44 organic acids were identified from QFPDD, including tartaric acid, quinic acid, citric acid, succinic acid, caffeic acid, vanillic acid isochlorogenic acid A, isochlorogenic acid B, isochlorogenic acid C, coumaroyltartaric acid, et al.

Saikosaponin A ( $\left.t_{R}=33.42 \mathrm{~min}\right)$, a triterpene saponins distributed in Radix Bupleuri, could be easily detected in the negative ion mode with a quasi-molecular ion $[\mathrm{M}-\mathrm{H}]^{-}$at $m / z 779.4589\left(\mathrm{C}_{42} \mathrm{H}_{67} \mathrm{O}_{13}\right)$. It was inclined to lose the sugar moiety to generate base peak ions $m / z$ of $617.4060\left[\mathrm{M}-\mathrm{H}-\mathrm{C}_{6} \mathrm{H}_{10} \mathrm{O}_{5}\right]^{-}, 471.3493[\mathrm{M}-\mathrm{H}-$ $\left.\mathrm{C}_{6} \mathrm{H}_{10} \mathrm{O}_{5}-\mathrm{C}_{6} \mathrm{H}_{10} \mathrm{O}_{4}\right]^{-}$and $179.0560\left[\mathrm{C}_{6} \mathrm{H}_{11} \mathrm{O}_{6}\right]^{-}$in $\mathrm{MS}^{2}$ spectra (Fig. 3C). Simultaneously the fragmentation signal of glucose was detected, its $\mathrm{MS} / \mathrm{MS}$ data were shown as $\mathrm{m} / \mathrm{z}$ of $161.0446\left[\mathrm{C}_{6} \mathrm{H}_{11} \mathrm{O}_{6}-\mathrm{H}_{2} \mathrm{O}\right]^{-}, 145.0495\left[\mathrm{C}_{6} \mathrm{H}_{11} \mathrm{O}_{6}-\mathrm{H}_{2} \mathrm{O}-\mathrm{O}\right]^{-}, 113.0495\left[\mathrm{C}_{6} \mathrm{H}_{11} \mathrm{O}_{6}-\mathrm{H}_{2} \mathrm{O}-\mathrm{C}_{2} \mathrm{H}_{4} \mathrm{O}_{2}\right]^{-}$and $101.0229\left[\mathrm{C}_{6} \mathrm{H}_{11} \mathrm{O}_{6}-\mathrm{H}_{2} \mathrm{O}-\mathrm{C}_{3} \mathrm{H}_{4} \mathrm{O}_{2}\right]^{-}$. Compound 374 listed in Table 1 could also be detected in the negative ion mode at the same Rt (at $33.42 \mathrm{~min}$ ) with the $\mathrm{m} / \mathrm{z}$ of $825.4652\left(\mathrm{C}_{42} \mathrm{H}_{67} \mathrm{O}_{13}\right)$, thus this compound was characterized as saikosaponin A. Glycyrrhizic acid ( $\left.t_{R}=31.86 \mathrm{~min}\right)$, a triterpene saponins distributed in Radix Glycyrrhizae Praeparata, could be detected in the negative ion mode with a quasi-molecular ion [M-H] $]^{-}$at $m / z$ of $821.3956\left(\mathrm{C}_{42} \mathrm{H}_{61} \mathrm{O}_{16}\right)$ in Fig. 3D. MS/MS fragmentation analysis demonstrated that this natural compound possessed loss of glucuronic acid group and glucosyl group, evidenced by the product ions of $m / z 645.3638\left[\mathrm{M}-\mathrm{H}-\mathrm{C}_{6} \mathrm{H}_{8} \mathrm{O}_{6}\right]^{-}, 469.3304[\mathrm{M}-$ $\left.\mathrm{H}-\mathrm{C}_{6} \mathrm{H}_{8} \mathrm{O}_{6}-\mathrm{C}_{6} \mathrm{H}_{8} \mathrm{O}_{6}\right]^{-}$. Simultaneously the fragmentation signal of glucuronic acid group and glucosyl group was detected, its MS/MS data were shown as $m / Z$ of $351.0563\left[\mathrm{M}-\mathrm{H}-\mathrm{C}_{30} \mathrm{H}_{46} \mathrm{O}_{4}\right]^{-}, 193.0341\left[\mathrm{M}-\mathrm{H}-\mathrm{C}_{30} \mathrm{H}_{46} \mathrm{O}_{4}-\mathrm{C}_{6} \mathrm{H}_{6} \mathrm{O}_{5}\right]^{-}$and $175.0232\left[\mathrm{M}-\mathrm{H}-\mathrm{C}_{30} \mathrm{H}_{46} \mathrm{O}_{4}-\mathrm{C}_{6} \mathrm{H}_{6} \mathrm{O}_{5}-\mathrm{H}_{2} \mathrm{O}\right]^{-}$. Compound 355 listed in Table 1 could also be detected in the negative ion mode at the same Rt (31.86 min) with the identical $\mathrm{m} / z$ of $821.3973\left(\mathrm{C}_{42} \mathrm{H}_{61} \mathrm{O}_{16}\right)$ and fragment ions, thus this compound was characterized as glycyrrhizic acid. Similar to the identification process above, a total of 71 triterpene saponins were identified from QFPDD, including saikosaponin B2, saikosaponin C, saikosaponin D, saikosaponin F, saikosaponin M, licorice saponin A3, licorice saponin B2, licorice Saponin G2, alisol A, alisol B, alisol C, alisol O, alisol A-23-acetate, alisol B-23-acetate, et al.

The remaining 88 kinds of chemical constituents were identified from QFPDD, including amino acids, nucleosides, sugars, phenols et al. they were tentatively characterized based on their retention times and mass spectrometric data, referring to the reference standards and previous literature. For example, MS ${ }^{2}$ spectra of compound 203 (molecular ion at $\mathrm{m} / \mathrm{z}\left[\mathrm{M}+\mathrm{H}^{+}\right.$133.0647) in Table 1 showed several characteristic fragment ions of $m / z 115.0538\left[\mathrm{M}+\mathrm{H}-\mathrm{H}_{2} \mathrm{O}\right]^{+}, \mathrm{m} / \mathrm{z}$ $105.0673[\mathrm{M}+\mathrm{H}-\mathrm{CO}]^{+}, m / z$ 86.10[M $\left.+\mathrm{H}-\mathrm{H}_{2} \mathrm{O}-\mathrm{C}_{2} \mathrm{H}_{5}\right]^{+}$. Thus, it corresponded to cinnamaldehyde by comparison with authentic standards and literature data [20]. Moreover, $\mathrm{MS}^{2}$ spectra of compound 322 (molecular ion at $\mathrm{m} / \mathrm{z}[\mathrm{M}+\mathrm{H}]+285.0752$ ) in Table 1 gave characteristic fragment ions of $m / z 270.0912$ [M $+\mathrm{H}$ $\left.\mathrm{CH}_{3}\right]^{+}, m / z 254.0943\left[\mathrm{M}+\mathrm{H}-\mathrm{CH}_{3} \mathrm{O}\right]^{+}, m / z 253.0897\left[\mathrm{M}+\mathrm{H}_{-} \mathrm{CH}_{3}-\mathrm{OH}\right]^{+}$and $m / z 225.0911\left[\mathrm{M}+\mathrm{H}_{-} \mathrm{CH}_{3} \mathrm{O}-\mathrm{CHO}^{+}\right.$. Then it was identified as batatasin I by comparison with authentic standards and literature data [21].

\subsection{Characterization of the absorbed chemical constituents in mice serum}

Page 21/47 


\subsubsection{Characterization of the absorbed prototype constituents in mice serum}

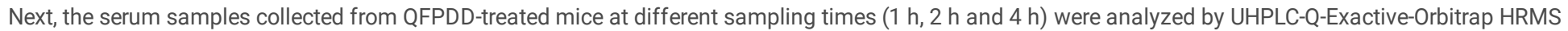

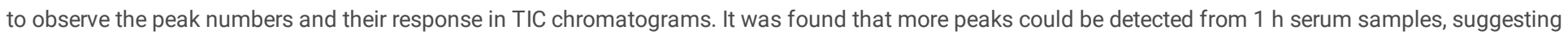

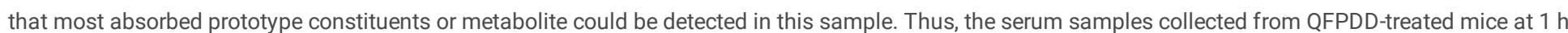

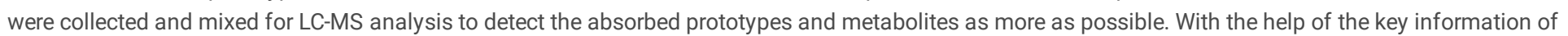

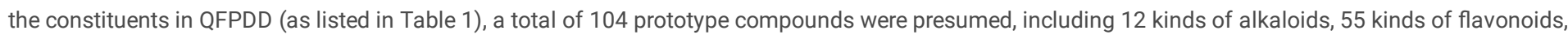

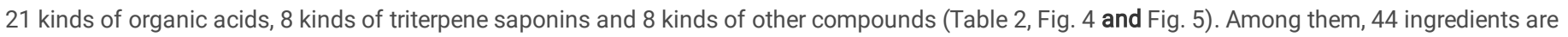

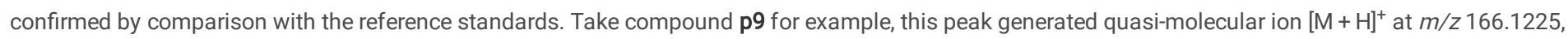

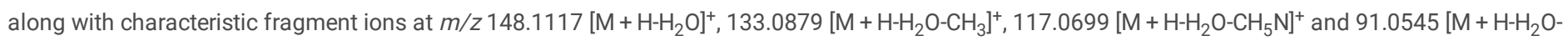

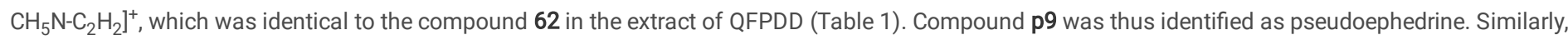

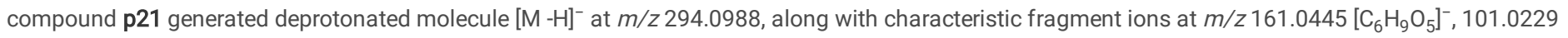

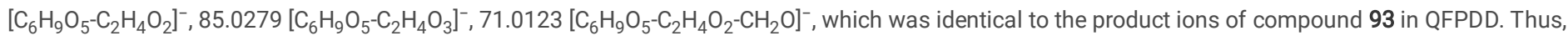
compound p21was characterized as prunasin. 
Table 2

Identification of prototype compounds of QFPDD in rat serum and multiple tissue by UHPLC-Q-Exactive Orbitr:

\begin{tabular}{|c|c|c|c|c|c|c|c|c|c|c|}
\hline \multirow[t]{2}{*}{ No. } & \multirow{2}{*}{$\begin{array}{l}\mathrm{RT} \\
/ \mathrm{min}\end{array}$} & \multirow{2}{*}{$\begin{array}{l}\text { lon } \\
\text { model }\end{array}$} & \multirow{2}{*}{$\begin{array}{l}\text { Measured } \\
\text { mass /Da }\end{array}$} & \multirow{2}{*}{$\begin{array}{l}\text { Calculated } \\
\text { mass /Da }\end{array}$} & \multirow{2}{*}{$\begin{array}{l}\text { Error } \\
\text { /ppm }\end{array}$} & \multirow{2}{*}{$\begin{array}{l}\text { Molecular } \\
\text { formula }\end{array}$} & \multirow{2}{*}{$\begin{array}{l}\text { Fragment } \\
\text { ions }\end{array}$} & \multirow[t]{2}{*}{ Identification } & \multicolumn{2}{|c|}{ Peak Area $\left(* 10^{6}\right)$} \\
\hline & & & & & & & & & Serum & Lung \\
\hline p1 & 0.90 & {$[\mathrm{M}-\mathrm{H}]^{-}$} & 149.0081 & 149.0092 & 0.239 & $\mathrm{C}_{4} \mathrm{H}_{6} \mathrm{O}_{6}$ & $\begin{array}{l}\text { 149.0080; } \\
\text { 87.0073; } \\
\text { 72.9916; } \\
\text { 59.0123 }\end{array}$ & Tartaric acid & 3.14 & 19.58 \\
\hline p2* & 0.92 & {$[\mathrm{M}-\mathrm{H}]^{-}$} & 191.0555 & 191.0561 & 2.279 & $\mathrm{C}_{7} \mathrm{H}_{12} \mathrm{O}_{6}$ & $\begin{array}{l}\text { 191.0189; } \\
\text { 111.0073; } \\
\text { 87.0072; } \\
85.0280 ; \\
57.0330\end{array}$ & Quinic acid & 88.17 & 406.21 \\
\hline p3* & 0.97 & $\begin{array}{l}{[\mathrm{M}+} \\
\mathrm{H}]^{+}\end{array}$ & 144.1019 & 144.1019 & -0.036 & $\mathrm{C}_{7} \mathrm{H}_{13} \mathrm{NO}_{2}$ & $\begin{array}{l}\text { 144.1017; } \\
84.0812 ; \\
56.0503\end{array}$ & Stachydrine & 673.88 & 606.92 \\
\hline $\mathrm{p} 4 *$ & 5.30 & $\begin{array}{l}{[\mathrm{M}+} \\
\mathrm{H}]^{+}\end{array}$ & 152.1070 & 152.1070 & -0.007 & $\mathrm{C}_{9} \mathrm{H}_{13} \mathrm{NO}$ & $\begin{array}{l}\text { 152.1068; } \\
\text { 134.0962; } \\
\text { 117.0700; } \\
90.9481 ; \\
56.0503\end{array}$ & Phenylpropanolamine & 7.48 & 41.12 \\
\hline p5* & 6.33 & $\begin{array}{l}{[\mathrm{M}+} \\
\mathrm{H}]^{+}\end{array}$ & 152.1070 & 152.1070 & 0.127 & $\mathrm{C}_{9} \mathrm{H}_{13} \mathrm{NO}$ & $\begin{array}{l}\text { 134.0963; } \\
\text { 117.0700; } \\
\text { 104.9636; } \\
\text { 91.0546; } \\
56.0503\end{array}$ & D-Demethyl pseudoephedrine & 8.27 & 21.68 \\
\hline p6* & 7.57 & {$[\mathrm{M}-\mathrm{H}]^{-}$} & 353.0883 & 353.0878 & 4.479 & $\mathrm{C}_{16} \mathrm{H}_{18} \mathrm{O}_{9}$ & $\begin{array}{l}353.0884 \\
191.0554 \\
179.0341 \\
135.0439 \\
85.0280\end{array}$ & Neochlorogenic acid & 1.47 & 10.28 \\
\hline $\mathrm{p} 7 *$ & 7.74 & $\begin{array}{l}{[\mathrm{M}+} \\
\mathrm{H}]^{+}\end{array}$ & 166.1227 & 166.1226 & 0.176 & $\mathrm{C}_{10} \mathrm{H}_{15} \mathrm{NO}$ & $\begin{array}{l}\text { 166.1223; } \\
\text { 148.1118; } \\
\text { 117.0699; } \\
\text { 91.0545; } \\
70.0658 ; \\
56.0502\end{array}$ & Ephedrine & 265.92 & 1579.7: \\
\hline p8 & 8.31 & $\begin{array}{l}{[\mathrm{M}+} \\
\mathrm{FA}-\mathrm{H}]^{-}\end{array}$ & 520.1677 & 520.1672 & 3.036 & $\mathrm{C}_{20} \mathrm{H}_{29} \mathrm{NO}_{12}$ & $\begin{array}{l}\text { 474.1601; } \\
\text { 312.1094; } \\
\text { 150.0549; } \\
\text { 101.0230; } \\
71.0124\end{array}$ & $\begin{array}{l}\text { D-a-[(6-O- } \beta \text {-D-glucopyranosyl- } \beta-D \text { - } \\
\text { glucopyranosyl)Oxy]Benzeneacetamide }\end{array}$ & 2.26 & 14.42 \\
\hline p9* & 8.66 & $\begin{array}{l}{[\mathrm{M}+} \\
\mathrm{H}]^{+}\end{array}$ & 166.1226 & 166.1226 & -0.064 & $\mathrm{C}_{10} \mathrm{H}_{15} \mathrm{NO}$ & $\begin{array}{l}\text { 166.1225; } \\
\text { 148.1117; } \\
\text { 133.0879; } \\
\text { 117.0699; } \\
\text { 91.0545; } \\
70.0657 ; \\
56.0502\end{array}$ & Pseudoephedrine & 75.77 & 266.35 \\
\hline p10 & 8.82 & $\begin{array}{l}{[\mathrm{M}+} \\
\mathrm{H}]^{+}\end{array}$ & 180.1382 & 180.1383 & -0.448 & $\mathrm{C}_{11} \mathrm{H}_{17} \mathrm{NO}$ & $\begin{array}{l}\text { 180.1380; } \\
\text { 162.1275; } \\
\text { 135.0803; } \\
\text { 117.0700; } \\
57.0343\end{array}$ & Methylephedrine & 107.64 & 375.19 \\
\hline p11 & 9.36 & {$[\mathrm{M}-\mathrm{H}]^{-}$} & 475.1463 & 475.1457 & 3.605 & $\mathrm{C}_{20} \mathrm{H}_{28} \mathrm{O}_{13}$ & $\begin{array}{l}\text { 475.1428; } \\
269.1033 ; \\
\text { 161.0445; } \\
\text { 113.0231; } \\
\text { 101.0230; } \\
71.0124\end{array}$ & L-Amygdalinic acid & 0.69 & 8.86 \\
\hline p12 & 11.85 & {$[\mathrm{M}-\mathrm{H}]^{-}$} & 475.1463 & 475.1457 & 3.605 & $\mathrm{C}_{20} \mathrm{H}_{28} \mathrm{O}_{13}$ & $\begin{array}{l}\text { 475.1468; } \\
314.1400 ; \\
269.1034 ; \\
181.0497 \\
135.0439 ; \\
101.0231\end{array}$ & D-Amygdalinic acid & 1.40 & 8.86 \\
\hline p13 & 11.96 & $\begin{array}{l}{[\mathrm{M}+} \\
\mathrm{H}]^{+}\end{array}$ & 137.1074 & 137.1073 & 0.182 & $\mathrm{C}_{8} \mathrm{H}_{12} \mathrm{~N}_{2}$ & $\begin{array}{l}\text { 137.1070; } \\
\text { 121.9662; } \\
\text { 91.0546; } \\
72.9377 ; \\
55.9352\end{array}$ & Ligustrazine & 0.06 & 2.42 \\
\hline
\end{tabular}




\begin{tabular}{|c|c|c|c|c|c|c|c|c|c|c|}
\hline \multirow[t]{2}{*}{ NO. } & \multirow{2}{*}{$\begin{array}{l}\mathrm{RT} \\
\text { /min }\end{array}$} & \multirow{2}{*}{$\begin{array}{l}\text { lon } \\
\text { model }\end{array}$} & \multirow{2}{*}{$\begin{array}{l}\text { Measured } \\
\text { mass /Da }\end{array}$} & \multirow{2}{*}{$\begin{array}{l}\text { Calculated } \\
\text { mass /Da }\end{array}$} & \multirow{2}{*}{$\begin{array}{l}\text { Error } \\
\text { /ppm }\end{array}$} & \multirow{2}{*}{$\begin{array}{l}\text { Molecular } \\
\text { formula }\end{array}$} & \multirow{2}{*}{$\begin{array}{l}\text { Fragment } \\
\text { ions }\end{array}$} & \multirow[t]{2}{*}{ Identification } & \multicolumn{2}{|c|}{ Peak Area $\left({ }^{*} 10^{6}\right)$} \\
\hline & & & & & & & & & Serum & Lung \\
\hline p14* & 12.43 & {$[\mathrm{M}-\mathrm{H}]^{-}$} & 353.0884 & 353.0878 & 4.734 & $\mathrm{C}_{16} \mathrm{H}_{18} \mathrm{O}_{9}$ & $\begin{array}{l}353.0884 \\
191.0551 ; \\
179.0331 \\
173.0445 \\
161.0231 \\
155.0320 \\
135.0442\end{array}$ & Chlorogenic acid & 8.40 & 65.98 \\
\hline p15* & 13.65 & {$[\mathrm{M}-\mathrm{H}]^{-}$} & 353.0884 & 353.0878 & 4.904 & $\mathrm{C}_{16} \mathrm{H}_{18} \mathrm{O}_{9}$ & $\begin{array}{l}353.0879 \\
251.0567 \\
191.0554 \\
179.0342 \\
173.0447 \\
135.0440 \\
93.0331\end{array}$ & Cryptochlorogenic acid & 2.18 & 20.53 \\
\hline p16 & 13.75 & {$[\mathrm{M}-\mathrm{H}]^{-}$} & 443.1933 & 443.1923 & 4.798 & $\mathrm{C}_{21} \mathrm{H}_{32} \mathrm{O}_{10}$ & $\begin{array}{l}443.1928 ; \\
375.0698 \\
245.0123 \\
195.0660 \\
165.0547 \\
113.0230 \\
101.0229\end{array}$ & $\begin{array}{l}\text { Dihydrophaseic acid } 4 \text { '-O- } \beta \text { - } \\
\text { glucopyranoside }\end{array}$ & 0.52 & 3.26 \\
\hline p17 & 13.79 & $\begin{array}{l}{[\mathrm{M}+} \\
\mathrm{FA}-\mathrm{H}]^{-}\end{array}$ & 502.1576 & 502.1566 & 4.070 & $\mathrm{C}_{20} \mathrm{H}_{27} \mathrm{NO}_{11}$ & $\begin{array}{l}323.0993 \\
245.0665 \\
221.0663 \\
179.0552 \\
161.0445 \\
119.0336 \\
101.0229\end{array}$ & Amygdalin & 28.57 & 203.76 \\
\hline p18 & 13.82 & $\begin{array}{l}{[\mathrm{M}+} \\
\mathrm{FA}-\mathrm{H}]^{-}\end{array}$ & 493.2292 & 493.2291 & 2.569 & $\mathrm{C}_{21} \mathrm{H}_{36} \mathrm{O}_{10}$ & $\begin{array}{l}\text { 447.2232; } \\
433.4934 \\
285.1710 \\
\text { 161.0448; } \\
\text { 143.0337; } \\
\text { 101.0230; } \\
89.0230\end{array}$ & Atractyloside A & 0.31 & 2.24 \\
\hline p19 & 14.01 & $\begin{array}{l}{[\mathrm{M}+} \\
\mathrm{FA}-\mathrm{H}]^{-}\end{array}$ & 477.1620 & 477.1614 & 3.610 & $\mathrm{C}_{19} \mathrm{H}_{28} \mathrm{O}_{11}$ & $\begin{array}{l}\text { 431.1563; } \\
269.1034 ; \\
\text { 161.0445; } \\
\text { 113.0231; } \\
\text { 101.0230; } \\
71.0123\end{array}$ & Benzyl- $\beta$-gentiobioside & 1.68 & 12.40 \\
\hline p20 & 14.03 & {$[\mathrm{M}-\mathrm{H}]^{-}$} & 431.1566 & 431.1559 & 4.179 & $\mathrm{C}_{19} \mathrm{H}_{28} \mathrm{O}_{11}$ & $\begin{array}{l}\text { 431.1562; } \\
311.0566 ; \\
\text { 191.0552; } \\
\text { 113.0230; } \\
99.0072 ; \\
59.0123\end{array}$ & Osmanthuside $\mathrm{H}$ or isomer & 0.90 & 6.03 \\
\hline p21 & 14.14 & {$[\mathrm{M}-\mathrm{H}]^{-}$} & 294.0988 & 294.0983 & 5.462 & $\mathrm{C}_{14} \mathrm{H}_{17} \mathrm{NO}_{6}$ & $\begin{array}{l}\text { 294.0988; } \\
\text { 161.0445; } \\
\text { 101.0229; } \\
\text { 85.0279; } \\
\text { 71.0123 }\end{array}$ & Prunasin & 8.31 & 7.16 \\
\hline $\mathrm{p} 22^{\star}$ & 15.27 & {$[\mathrm{M}-\mathrm{H}]^{-}$} & 515.1197 & 515.1195 & 2.499 & $\mathrm{C}_{25} \mathrm{H}_{24} \mathrm{O}_{12}$ & $\begin{array}{l}485.9193 \\
434.3426 \\
353.0880 \\
285.1937 \\
191.0553 \\
161.0233 \\
135.0438\end{array}$ & 1,3-O-Dicaffeoylquinic acid & 0.09 & 2.17 \\
\hline p23 & 15.39 & $\begin{array}{l}{[\mathrm{M}+} \\
\mathrm{H}]^{+}\end{array}$ & 303.0500 & 303.0499 & 0.201 & $\mathrm{C}_{15} \mathrm{H}_{10} \mathrm{O}_{7}$ & $\begin{array}{l}303.0493 \\
283.1068 \\
257.0439 \\
229.0490 \\
207.0285 \\
139.0386 \\
111.0078\end{array}$ & Herbacetin & 1.19 & 5.52 \\
\hline p24 & 16.20 & {$[\mathrm{M}-\mathrm{H}]^{-}$} & 327.1091 & 327.1085 & 5.154 & $\mathrm{C}_{15} \mathrm{H}_{20} \mathrm{O}_{9}$ & $\begin{array}{l}311.9916 ; \\
205.0349 ; \\
165.0548 ; \\
143.0338 ; \\
125.0231 ; \\
121.0646 ; \\
81.0331\end{array}$ & Dihydrocinnacasside & 6.18 & 11.36 \\
\hline
\end{tabular}




\begin{tabular}{|c|c|c|c|c|c|c|c|c|c|c|}
\hline \multirow[t]{2}{*}{ No. } & \multirow{2}{*}{$\begin{array}{l}\mathrm{RT} \\
/ \mathrm{min}\end{array}$} & \multirow{2}{*}{$\begin{array}{l}\text { lon } \\
\text { model }\end{array}$} & \multirow{2}{*}{$\begin{array}{l}\text { Measured } \\
\text { mass /Da }\end{array}$} & \multirow{2}{*}{$\begin{array}{l}\text { Calculated } \\
\text { mass /Da }\end{array}$} & \multirow{2}{*}{$\begin{array}{l}\text { Error } \\
\text { /ppm }\end{array}$} & \multirow{2}{*}{$\begin{array}{l}\text { Molecular } \\
\text { formula }\end{array}$} & \multirow{2}{*}{$\begin{array}{l}\text { Fragment } \\
\text { ions }\end{array}$} & \multirow[t]{2}{*}{ Identification } & \multicolumn{2}{|c|}{ Peak Area $\left(* 10^{6}\right)$} \\
\hline & & & & & & & & & Serum & Lung \\
\hline p25 & 17.00 & {$[\mathrm{M}-\mathrm{H}]^{-}$} & 593.1520 & 593.1512 & 3.770 & $\mathrm{C}_{27} \mathrm{H}_{30} \mathrm{O}_{15}$ & $\begin{array}{l}\text { 593.1522; } \\
503.1204 ; \\
473.1093 ; \\
383.0779 ; \\
353.0672 ; \\
271.0611 \\
191.0342\end{array}$ & Vicenin-li & 1.43 & 8.82 \\
\hline p26 & 17.39 & {$[\mathrm{M}-\mathrm{H}]^{-}$} & 463.0891 & 463.0882 & 4.206 & $\mathrm{C}_{21} \mathrm{H}_{20} \mathrm{O}_{12}$ & $\begin{array}{l}\text { 463.0892; } \\
341.0670 \\
300.0278 \\
271.0252 \\
\text { 178.9975; } \\
\text { 139.0388; } \\
89.0230\end{array}$ & Carthamidin-7-O-glucuronide & 18.23 & 4.24 \\
\hline p27* & 17.43 & {$[\mathrm{M}-\mathrm{H}]^{-}$} & 193.0495 & 195.0652 & 0.485 & $\mathrm{C}_{10} \mathrm{H}_{10} \mathrm{O}_{4}$ & $\begin{array}{l}\text { 193.0493; } \\
177.0542 ; \\
163.0387 ; \\
145.0281 ; \\
135.0439 ; \\
117.0335 ; \\
89.0388\end{array}$ & Ferulic acid & 15.28 & 4.94 \\
\hline p28 & 17.59 & {$[\mathrm{M}-\mathrm{H}]^{-}$} & 623.1632 & 623.1618 & 4.090 & $\mathrm{C}_{28} \mathrm{H}_{32} \mathrm{O}_{16}$ & $\begin{array}{l}\text { 623.1628; } \\
\text { 533.1307; } \\
\text { 503.1204; } \\
\text { 463.0891; } \\
\text { 413.0885; } \\
383.0779 \\
312.0631\end{array}$ & Isorhamnetin-3-O-rutinoside & 0.15 & 3.16 \\
\hline p29 & 17.88 & $\begin{array}{l}{[\mathrm{M}+} \\
\mathrm{H}]^{+}\end{array}$ & 625.1758 & 625.1763 & -0.850 & $\mathrm{C}_{28} \mathrm{H}_{32} \mathrm{O}_{16}$ & $\begin{array}{l}\text { 581.2217; } \\
\text { 419.1717; } \\
\text { 404.1479; } \\
373.1295 \\
233.0816 \\
202.0629 \\
\text { 153.0546 }\end{array}$ & 4'-Methoxy-lucenin-2 & 0.10 & 3.11 \\
\hline p30 & 18.11 & {$[\mathrm{M}-\mathrm{H}]^{-}$} & 581.2245 & 581.2240 & 2.792 & $\mathrm{C}_{28} \mathrm{H}_{38} \mathrm{O}_{13}$ & $\begin{array}{l}\text { 581.2239; } \\
\text { 53.1824; } \\
\text { 419.1716; } \\
\text { 404.1482; } \\
373.1298 \\
\text { 355.1193; } \\
223.0818\end{array}$ & (+)-Lyoniresinol 9'-O-glucoside & 0.13 & 2.06 \\
\hline p31 & 18.17 & {$[\mathrm{M}-\mathrm{H}]^{-}$} & 295.0463 & 295.0459 & 4.800 & $\mathrm{C}_{13} \mathrm{H}_{12} \mathrm{O}_{8}$ & $\begin{array}{l}295.1540 ; \\
259.0247 \\
221.0816 \\
189.0550 \\
163.0760 \\
143.0492 \\
121.0283\end{array}$ & Coumaroyltartaric acid & 1.99 & 5.88 \\
\hline p32* & 18.21 & {$[\mathrm{M}-\mathrm{H}]^{-}$} & 137.0233 & 137.0244 & -0.515 & $\mathrm{C}_{7} \mathrm{H}_{6} \mathrm{O}_{3}$ & $\begin{array}{l}\text { 137.0231; } \\
93.0331 ; \\
84.8779 \\
70.7439\end{array}$ & Salicylic acid & 295.38 & 94.00 \\
\hline p33* & 18.39 & $\begin{array}{l}{[\mathrm{M}+} \\
\mathrm{H}]^{+}\end{array}$ & 433.1130 & 433.1129 & 0.131 & $\mathrm{C}_{21} \mathrm{H}_{20} \mathrm{O}_{10}$ & $\begin{array}{l}\text { 433.1108; } \\
\text { 415.1017; } \\
397.0912 ; \\
337.0700 ; \\
313.0701 ; \\
271.0596 ; \\
206.0546\end{array}$ & Isovitexin & 2.84 & 0.00 \\
\hline p34* & 18.57 & {$[\mathrm{M}-\mathrm{H}]^{-}$} & 417.1197 & 417.1191 & 4.103 & $\mathrm{C}_{21} \mathrm{H}_{22} \mathrm{O}_{9}$ & $\begin{array}{l}\text { 417.1188; } \\
255.0664 ; \\
191.0560 ; \\
153.0183 \\
\text { 153.0183; } \\
\text { 135.0076; } \\
119.0488\end{array}$ & Liquiritin & 2.12 & 99.99 \\
\hline p35 & 18.82 & {$[\mathrm{M}-\mathrm{H}]^{-}$} & 595.1672 & 595.1668 & 2.409 & $\mathrm{C}_{27} \mathrm{H}_{32} \mathrm{O}_{15}$ & $\begin{array}{l}\text { 595.1689; } \\
\text { 435.0936; } \\
315.0723 ; \\
271.0615 \\
177.0186 \\
151.0026 \\
137.0233\end{array}$ & Neoeriocitrin & 0.08 & 11.56 \\
\hline
\end{tabular}




\begin{tabular}{|c|c|c|c|c|c|c|c|c|c|c|}
\hline \multirow[t]{2}{*}{ No. } & \multirow{2}{*}{$\begin{array}{l}\mathrm{RT} \\
/ \mathrm{min}\end{array}$} & \multirow{2}{*}{$\begin{array}{l}\text { lon } \\
\text { model }\end{array}$} & \multirow{2}{*}{$\begin{array}{l}\text { Measured } \\
\text { mass /Da }\end{array}$} & \multirow{2}{*}{$\begin{array}{l}\text { Calculated } \\
\text { mass /Da }\end{array}$} & \multirow{2}{*}{$\begin{array}{l}\text { Error } \\
\text { /ppm }\end{array}$} & \multirow{2}{*}{$\begin{array}{l}\text { Molecular } \\
\text { formula }\end{array}$} & \multirow{2}{*}{$\begin{array}{l}\text { Fragment } \\
\text { ions }\end{array}$} & \multirow[t]{2}{*}{ Identification } & \multicolumn{2}{|c|}{ Peak Area $\left({ }^{*} 10^{6}\right)$} \\
\hline & & & & & & & & & Serum & Lung \\
\hline p36 & 18.84 & $\begin{array}{l}{[\mathrm{M}+} \\
\mathrm{H}]^{+}\end{array}$ & 366.1911 & 366.1911 & -0.038 & $\mathrm{C}_{19} \mathrm{H}_{27} \mathrm{NO}_{6}$ & $\begin{array}{l}366.1905 ; \\
348.1799 \\
320.1843 \\
242.8689 \\
168.1017 \\
150.0912 \\
122.0600\end{array}$ & Senkirkine /isomer & 9.05 & 36.41 \\
\hline p37 & 18.86 & {$[\mathrm{M}-\mathrm{H}]^{-}$} & 741.2265 & 741.2248 & 3.838 & $\mathrm{C}_{33} \mathrm{H}_{42} \mathrm{O}_{19}$ & $\begin{array}{l}\text { 741.2263; } \\
\text { 621.1686; } \\
\text { 417.1567; } \\
\text { 353.1033; } \\
271.0615 ; \\
181.0499 \\
151.0062\end{array}$ & Naringenin-7-O-triglycoside & 0.37 & 8.96 \\
\hline p38 & 19.28 & {$[\mathrm{M}-\mathrm{H}]^{-}$} & 595.1679 & 595.1668 & 3.534 & $\mathrm{C}_{27} \mathrm{H}_{32} \mathrm{O}_{15}$ & $\begin{array}{l}\text { 595.1689; } \\
435.0936 \\
315.0723 \\
271.0615 \\
177.0186 \\
151.0026 \\
137.0233\end{array}$ & Eriocitrin & 0.09 & 46.34 \\
\hline p39 & 19.50 & {$[\mathrm{M}-\mathrm{H}]^{-}$} & 547.1466 & 547.1457 & 3.532 & $\mathrm{C}_{26} \mathrm{H}_{28} \mathrm{O}_{13}$ & $\begin{array}{l}547.1465 ; \\
487.1251 ; \\
457.1147 ; \\
427.1038 ; \\
367.0827 \\
337.0723 \\
255.0665\end{array}$ & chrysin-6-C-arabinosyl-8- $C$-glucoside & 6.88 & 68.09 \\
\hline $\mathrm{p} 40^{*}$ & 19.55 & {$[\mathrm{M}-\mathrm{H}]^{-}$} & 461.0734 & 461.0725 & 4.203 & $\mathrm{C}_{21} \mathrm{H}_{18} \mathrm{O}_{12}$ & $\begin{array}{l}461.1097 \\
446.0860 \\
341.0640 \\
298.0486 \\
255.0664 \\
167.0341\end{array}$ & Scutellarin & 1.28 & 6.20 \\
\hline $\mathrm{p} 41^{*}$ & 19.61 & {$[\mathrm{M}-\mathrm{H}]^{-}$} & 461.1098 & 461.1089 & 4.320 & $\mathrm{C}_{22} \mathrm{H}_{22} \mathrm{O}_{11}$ & $\begin{array}{l}461.1094 \\
371.0776 \\
330.0383 \\
\text { 198.0483; } \\
\text { 185.0407; } \\
129.0179\end{array}$ & Tectoridin & 0.31 & 38.15 \\
\hline $\mathrm{p} 42$ & 19.72 & {$[\mathrm{M}-\mathrm{H}]^{-}$} & 771.2364 & 771.2353 & 2.814 & $\mathrm{C}_{34} \mathrm{H}_{44} \mathrm{O}_{20}$ & $\begin{array}{l}\text { 771.2362; } \\
607.1677 \\
489.1410 \\
301.0719 \\
286.0484 \\
125.0231\end{array}$ & Alpha-glucosyl hesperidin & 0.23 & 6.42 \\
\hline $\mathrm{p} 43^{*}$ & 19.74 & {$[\mathrm{M}-\mathrm{H}]^{-}$} & 515.1202 & 515.1195 & 3.451 & $\mathrm{C}_{25} \mathrm{H}_{24} \mathrm{O}_{12}$ & $\begin{array}{l}353.0883 \\
191.0554 \\
173.0447 \\
135.0439 \\
93.0330\end{array}$ & Isochlorogenic acid B & 0.89 & 91.68 \\
\hline p44 & 19.83 & $\begin{array}{l}{[\mathrm{M}+} \\
\mathrm{H}]^{+}\end{array}$ & 493.1317 & 493.1343 & -4.811 & $\mathrm{C}_{23} \mathrm{H}_{24} \mathrm{O}_{12}$ & $\begin{array}{l}\text { 493.1729; } \\
\text { 417.0955; } \\
375.0841 ; \\
331.0807 ; \\
316.0572 ; \\
245.1202 ; \\
197.0441\end{array}$ & Iristectorin A & 0.68 & 10.92 \\
\hline p45 & 19.89 & $\begin{array}{l}{[\mathrm{M}+} \\
\mathrm{H}]^{+}\end{array}$ & 465.1031 & 465.1028 & 0.726 & $\mathrm{C}_{21} \mathrm{H}_{20} \mathrm{O}_{12}$ & 465.1031 & Hyperin & 0.12 & 37.73 \\
\hline $\mathrm{p} 46$ & 19.98 & {$[\mathrm{M}-\mathrm{H}]^{-}$} & 577.1570 & 577.1563 & 3.219 & $\mathrm{C}_{27} \mathrm{H}_{30} \mathrm{O}_{14}$ & $\begin{array}{l}\text { 577.1570; } \\
\text { 457.1139; } \\
413.0885 ; \\
341.0673 ; \\
293.0461 ; \\
269.0458 \\
59.0124\end{array}$ & Isoviolanthin & 2.38 & 15.29 \\
\hline $\mathrm{p} 47$ & 19.99 & $\begin{array}{l}{[\mathrm{M}+} \\
\mathrm{H}]^{+}\end{array}$ & 449.1078 & 449.1078 & -0.173 & $\mathrm{C}_{21} \mathrm{H}_{20} \mathrm{O}_{11}$ & $\begin{array}{l}426.0634 \\
398.0688 \\
369.0294 \\
353.0887 \\
338.0652 \\
165.0909\end{array}$ & Naringenin-7-O-glucuronide & 39.81 & 6.91 \\
\hline
\end{tabular}




\begin{tabular}{|c|c|c|c|c|c|c|c|c|c|c|}
\hline \multirow[t]{2}{*}{ NO. } & \multirow{2}{*}{$\begin{array}{l}\mathrm{RT} \\
/ \mathrm{min}\end{array}$} & \multirow{2}{*}{$\begin{array}{l}\text { lon } \\
\text { model }\end{array}$} & \multirow{2}{*}{$\begin{array}{l}\text { Measured } \\
\text { mass /Da }\end{array}$} & \multirow{2}{*}{$\begin{array}{l}\text { Calculated } \\
\text { mass } / \mathrm{Da}\end{array}$} & \multirow{2}{*}{$\begin{array}{l}\text { Error } \\
\text { /ppm }\end{array}$} & \multirow{2}{*}{$\begin{array}{l}\text { Molecular } \\
\text { formula }\end{array}$} & \multirow{2}{*}{$\begin{array}{l}\text { Fragment } \\
\text { ions }\end{array}$} & \multirow[t]{2}{*}{ Identification } & \multicolumn{2}{|c|}{ Peak Area $\left({ }^{*} 10^{6}\right)$} \\
\hline & & & & & & & & & Serum & Lung \\
\hline p48* & 19.99 & {$[\mathrm{M}-\mathrm{H}]^{-}$} & 463.0892 & 463.0882 & 4.530 & $\mathrm{C}_{21} \mathrm{H}_{20} \mathrm{O}_{12}$ & $\begin{array}{l}\text { 463.0892; } \\
\text { 341.0670; } \\
\text { 300.0278; } \\
271.0252 ; \\
\text { 178.9975; } \\
\text { 139.0388; } \\
89.0230\end{array}$ & Isoquercitrin & 0.61 & 10.56 \\
\hline p49* & 20.01 & {$[\mathrm{M}-\mathrm{H}]^{-}$} & 609.1467 & 609.1461 & 2.822 & $\mathrm{C}_{27} \mathrm{H}_{30} \mathrm{O}_{16}$ & $\begin{array}{l}609.1473 \\
487.1224 \\
431.0981 \\
300.0278 \\
269.0461 \\
178.9979\end{array}$ & Rutin & 0.16 & 20.92 \\
\hline $\mathrm{p} 50^{*}$ & 20.03 & {$[\mathrm{M}-\mathrm{H}]^{-}$} & 515.1202 & 515.1195 & 3.451 & $\mathrm{C}_{25} \mathrm{H}_{24} \mathrm{O}_{12}$ & $\begin{array}{l}\text { 515.1160; } \\
353.0884 ; \\
191.0554 ; \\
179.0337 \\
161.0234\end{array}$ & Isochlorogenic acid A & 0.87 & 10.05 \\
\hline p51 & 20.18 & $\begin{array}{l}{[\mathrm{M}+} \\
\mathrm{H}]^{+}\end{array}$ & 449.1078 & 449.1078 & -0.173 & $\mathrm{C}_{21} \mathrm{H}_{20} \mathrm{O}_{11}$ & $\begin{array}{l}426.0634 \\
398.0688 \\
369.0294 \\
353.0887 \\
338.0652 \\
165.0909\end{array}$ & Naringenin-4'-O-glucuronide & 39.81 & 2.13 \\
\hline p52 & 20.57 & {$[\mathrm{M}-\mathrm{H}]^{-}$} & 547.1466 & 547.1457 & 3.532 & $\mathrm{C}_{26} \mathrm{H}_{28} \mathrm{O}_{13}$ & $\begin{array}{l}\text { 547.1463; } \\
\text { 457.1144; } \\
\text { 427.1038; } \\
367.0829 ; \\
337.0724 ; \\
300.0280 ; \\
191.0339\end{array}$ & chrysin-6-C-glucosyl-8-C-arabinoside & 2.68 & 42.87 \\
\hline p53* & 20.73 & {$[\mathrm{M}-\mathrm{H}]^{-}$} & 579.1728 & 579.1719 & 3.381 & $\mathrm{C}_{27} \mathrm{H}_{32} \mathrm{O}_{14}$ & $\begin{array}{l}579.1724 ; \\
459.1160 \\
415.1048 \\
313.0718 \\
271.0614 ; \\
235.0247 \\
151.0026\end{array}$ & Naringin & 2.44 & $1402.4 \imath$ \\
\hline p54* & 20.82 & $\begin{array}{l}{[\mathrm{M}+} \\
\mathrm{H}]^{+}\end{array}$ & 229.0860 & 229.0859 & 0.389 & $\mathrm{C}_{14} \mathrm{H}_{12} \mathrm{O}_{3}$ & $\begin{array}{l}229.0855 ; \\
211.0750 ; \\
183.0803 ; \\
145.0648 ; \\
135.0439 ; \\
119.0492 ; \\
107.0494\end{array}$ & Resveratrol & 1.04 & 0.29 \\
\hline p55 & 20.88 & {$[\mathrm{M}-\mathrm{H}]^{-}$} & 581.1899 & 581.1876 & 5.950 & $\mathrm{C}_{27} \mathrm{H}_{34} \mathrm{O}_{14}$ & 581.1895 & $\begin{array}{l}2,4,6 \text {-Trihydroxydihydrochalcone3 }-C \text { - } \\
\beta \text {-D-glucoside- } 6 \text { - } O-\beta \text {-D-glucoside }\end{array}$ & 0.31 & 1.53 \\
\hline p56* & 20.92 & $\begin{array}{l}{[\mathrm{M}+} \\
\mathrm{H}]^{+}\end{array}$ & 493.1342 & 493.1343 & 0.279 & $\mathrm{C}_{23} \mathrm{H}_{24} \mathrm{O}_{12}$ & $\begin{array}{l}\text { 493.2296; } \\
373.0909 ; \\
331.0807 ; \\
271.0594 ; \\
177.0545\end{array}$ & Iristectorin B & 0.21 & 39.56 \\
\hline p57* & 21.21 & {$[\mathrm{M}-\mathrm{H}]^{-}$} & 609.1837 & 609.1825 & 3.699 & $\mathrm{C}_{28} \mathrm{H}_{34} \mathrm{O}_{15}$ & $\begin{array}{l}\text { 609.1832; } \\
489.1412 ; \\
343.0831 ; \\
301.0721 ; \\
217.0510\end{array}$ & Hesperidin & 1.10 & 246.73 \\
\hline p58* & 21.38 & {$[\mathrm{M}-\mathrm{H}]^{-}$} & 515.1202 & 515.1195 & 3.452 & $\mathrm{C}_{25} \mathrm{H}_{24} \mathrm{O}_{12}$ & $\begin{array}{l}515.1160 ; \\
353.0884 ; \\
255.0663 \\
191.0554 \\
173.0447 \\
161.0234\end{array}$ & Isochlorogenic acid C & 0.23 & 66.60 \\
\hline p59* & 21.58 & {$[\mathrm{M}-\mathrm{H}]^{-}$} & 609.1834 & 609.1825 & 3.305 & $\mathrm{C}_{28} \mathrm{H}_{34} \mathrm{O}_{15}$ & $\begin{array}{l}609.1829 \\
489.1410 \\
403.1042 \\
343.0825 \\
301.0720 \\
286.0485\end{array}$ & Neohesperidin & 1.98 & 983.38 \\
\hline
\end{tabular}




\begin{tabular}{|c|c|c|c|c|c|c|c|c|c|c|}
\hline \multirow[t]{2}{*}{ NO. } & \multirow{2}{*}{$\begin{array}{l}\mathrm{RT} \\
\text { /min }\end{array}$} & \multirow{2}{*}{$\begin{array}{l}\text { Ion } \\
\text { model }\end{array}$} & \multirow{2}{*}{$\begin{array}{l}\text { Measured } \\
\text { mass /Da }\end{array}$} & \multirow{2}{*}{$\begin{array}{l}\text { Calculated } \\
\text { mass /Da }\end{array}$} & \multirow{2}{*}{$\begin{array}{l}\text { Error } \\
\text { /ppm }\end{array}$} & \multirow{2}{*}{$\begin{array}{l}\text { Molecular } \\
\text { formula }\end{array}$} & \multirow{2}{*}{$\begin{array}{l}\text { Fragment } \\
\text { ions }\end{array}$} & \multirow[t]{2}{*}{ Identification } & \multicolumn{2}{|c|}{ Peak Area $\left({ }^{*} 10^{6}\right)$} \\
\hline & & & & & & & & & Serum & Lung \\
\hline p60* & 21.92 & {$[\mathrm{M}-\mathrm{H}]^{-}$} & 255.0660 & 255.0663 & 5.715 & $\mathrm{C}_{15} \mathrm{H}_{12} \mathrm{O}_{4}$ & $\begin{array}{l}255.0665 \\
213.4413 \\
153.0183 \\
135.0076 \\
\text { 119.0490; } \\
91.0175\end{array}$ & Liquiritigenin & 0.89 & 0.00 \\
\hline p61 & 22.05 & {$[\mathrm{M}-\mathrm{H}]^{-}$} & 201.0188 & 201.0193 & 2.959 & $\mathrm{C}_{11} \mathrm{H}_{6} \mathrm{O}_{4}$ & $\begin{array}{l}\text { 201.0676; } \\
\text { 183.1021; } \\
\text { 169.0399; } \\
\text { 144.0443; } \\
\text { 131.03664; } \\
\text { 116.0494; } \\
\text { 116.0493 }\end{array}$ & Xanthotoxol & 0.05 & 11.43 \\
\hline p62* & 22.06 & {$[\mathrm{M}-\mathrm{H}]^{-}$} & 417.1199 & 417.1191 & 4.462 & $\mathrm{C}_{21} \mathrm{H}_{22} \mathrm{O}_{9}$ & $\begin{array}{l}\text { 417.1203; } \\
358.4896 \\
297.0761 \\
255.0663 \\
148.0155 \\
135.0076\end{array}$ & Isoliquiritin & 0.27 & 13.09 \\
\hline p63 & 22.12 & {$[\mathrm{M}-\mathrm{H}]^{-}$} & 443.0950 & 443.0983 & -7.6 & $\mathrm{C}_{22} \mathrm{H}_{20} \mathrm{O}_{10}$ & $\begin{array}{l}267.0667 \\
252.0429 \\
227.0188 \\
175.0238\end{array}$ & Formononetin-glucuronide & 4.00 & 1.65 \\
\hline p64 & 22.26 & $\begin{array}{l}{[\mathrm{M}+} \\
\mathrm{FA}-\mathrm{H}]^{-}\end{array}$ & 475.1253 & 475.1267 & 3.898 & $\mathrm{C}_{22} \mathrm{H}_{22} \mathrm{O}_{9}$ & $\begin{array}{l}329.0312 \\
299.0560 \\
267.0666 \\
151.0426 \\
151.0027\end{array}$ & Ononin & 0.10 & 10.49 \\
\hline p65 & 22.39 & $\begin{array}{l}{[\mathrm{M}+} \\
\mathrm{H}]^{+}\end{array}$ & 532.2408 & 532.2402 & 1.222 & $\mathrm{C}_{25} \mathrm{H}_{33} \mathrm{~N}_{5} \mathrm{O}_{8}$ & $\begin{array}{l}479.2282 ; \\
361.0911 ; \\
346.0677 ; \\
273.0752 ; \\
180.1016\end{array}$ & Asterinin A & 0.85 & 1.24 \\
\hline p66* & 22.84 & {$[\mathrm{M}-\mathrm{H}]^{-}$} & 445.0783 & 445.0776 & 3.982 & $\mathrm{C}_{21} \mathrm{H}_{18} \mathrm{O}_{11}$ & $\begin{array}{l}355.0813 ; \\
269.0457 \\
243.0666 ; \\
175 ; 0241 \\
113.0230\end{array}$ & Baicalin & 217.59 & 974.47 \\
\hline p67 & 22.85 & $\begin{array}{l}{[\mathrm{M}+} \\
\mathrm{H}]^{+}\end{array}$ & 287.0548 & 287.0550 & -0.817 & $\mathrm{C}_{15} \mathrm{H}_{10} \mathrm{O}_{6}$ & $\begin{array}{l}287.0545 ; \\
269.0440 ; \\
241.0492 ; \\
225.0545 ; \\
197.0594 ; \\
139.0022 ; \\
103.0546\end{array}$ & Luteolin & 13.44 & 65.73 \\
\hline p68 & 22.96 & {$[\mathrm{M}-\mathrm{H}]^{-}$} & 263.1292 & 263.1289 & 5.299 & $\mathrm{C}_{15} \mathrm{H}_{20} \mathrm{O}_{4}$ & $\begin{array}{l}263.0962 ; \\
219.1387 \\
204.1150 ; \\
151.0754 ; \\
139.0753 \\
79.9559\end{array}$ & $(+)$-Abscisic acid & 1.68 & 4.82 \\
\hline p69 & 23.13 & $\begin{array}{l}{[\mathrm{M}+} \\
\mathrm{H}]^{+}\end{array}$ & 361.0916 & 361.0930 & -0.675 & $\mathrm{C}_{18} \mathrm{H}_{16} \mathrm{O}_{8}$ & $\begin{array}{l}361.0910 ; \\
346.0677 ; \\
301.0700 ; \\
286.0466 ; \\
219.1172 ; \\
174.0911 ; \\
117.0700\end{array}$ & Isoirigenin & 56.14 & 91.11 \\
\hline p70 & 23.92 & {$[\mathrm{M}-\mathrm{H}]^{-}$} & 271.0616 & 271.0612 & 5.350 & $\mathrm{C}_{15} \mathrm{H}_{12} \mathrm{O}_{5}$ & $\begin{array}{l}271.0614 \\
227.0712 \\
177.0184 \\
151.0025 \\
119.0489 \\
93.03331\end{array}$ & Naringenin & 1.33 & 102.41 \\
\hline p71 & 24.00 & {$[\mathrm{M}-\mathrm{H}]^{-}$} & 445.1147 & 445.1140 & 3.969 & $\mathrm{C}_{22} \mathrm{H}_{22} \mathrm{O}_{10}$ & $\begin{array}{l}445.1147 \\
430.0911 \\
325.0717 \\
283.0615 \\
268.0374 \\
113.0230\end{array}$ & Wogonin-5-O- $\beta-D$-glucoside & 7.33 & 1.44 \\
\hline
\end{tabular}




\begin{tabular}{|c|c|c|c|c|c|c|c|c|c|c|}
\hline \multirow[t]{2}{*}{ NO. } & \multirow{2}{*}{$\begin{array}{l}\mathrm{RT} \\
\text { /min }\end{array}$} & \multirow{2}{*}{$\begin{array}{l}\text { lon } \\
\text { model }\end{array}$} & \multirow{2}{*}{$\begin{array}{l}\text { Measured } \\
\text { mass /Da }\end{array}$} & \multirow{2}{*}{$\begin{array}{l}\text { Calculated } \\
\text { mass /Da }\end{array}$} & \multirow{2}{*}{$\begin{array}{l}\text { Error } \\
\text { /ppm }\end{array}$} & \multirow{2}{*}{$\begin{array}{l}\text { Molecular } \\
\text { formula }\end{array}$} & \multirow{2}{*}{$\begin{array}{l}\text { Fragment } \\
\text { ions }\end{array}$} & \multirow[t]{2}{*}{ Identification } & \multicolumn{2}{|c|}{ Peak Area $\left({ }^{*} 10^{6}\right)$} \\
\hline & & & & & & & & & Serum & Lung \\
\hline p72 & 24.28 & {$[\mathrm{M}-\mathrm{H}]^{-}$} & 429.0832 & 429.0827 & 3.582 & $\mathrm{C}_{21} \mathrm{H}_{18} \mathrm{O}_{10}$ & $\begin{array}{l}429.2076 \\
342.4299 \\
253.0507 \\
223.1342 \\
175.0239 \\
113.0229 \\
85.0279\end{array}$ & Chrysin-7-O- $\beta$-D-glucuronide & 11.43 & 65.77 \\
\hline p73 & 24.28 & {$[\mathrm{M}-\mathrm{H}]^{-}$} & 461.1099 & 461.1089 & 4.451 & $\mathrm{C}_{22} \mathrm{H}_{22} \mathrm{O}_{11}$ & $\begin{array}{l}461.1095 \\
440.0857 \\
299.0558 \\
283.0251 \\
268.0379 \\
211.0398 \\
113.0230\end{array}$ & $\begin{array}{l}\text { (2S)-5,7-Dihydroxy-6- } \\
\text { methoxyflavanone7-O- } \beta \text {-D-glucuronide }\end{array}$ & 9.36 & 20.24 \\
\hline p74 & 24.40 & {$[\mathrm{M}-\mathrm{H}]^{-}$} & 473.0732 & 473.0725 & 3.715 & $\mathrm{C}_{22} \mathrm{H}_{18} \mathrm{O}_{12}$ & $\begin{array}{l}473.1093 \\
413.0858 \\
349.1303 \\
307.0831 \\
268.0380 \\
\text { 165.0547; } \\
59.0123\end{array}$ & Cichoric acid & 0.22 & 0.57 \\
\hline p75* & 24.67 & {$[\mathrm{M}-\mathrm{H}]^{-}$} & 459.0940 & 459.0933 & 3.991 & $\mathrm{C}_{22} \mathrm{H}_{20} \mathrm{O}_{11}$ & $\begin{array}{l}299.0563 \\
283.0613 \\
268.0379 \\
175.0238 \\
113.0230\end{array}$ & Oroxylin A 7-O-glucuronide & 33.80 & 290.88 \\
\hline p76 & 24.74 & $\begin{array}{l}{[\mathrm{M}+} \\
\mathrm{H}]^{+}\end{array}$ & 301.0706 & 301.0711 & -0.248 & $\mathrm{C}_{16} \mathrm{H}_{12} \mathrm{O}_{6}$ & $\begin{array}{l}301.1040 \\
269.0439 \\
197.0448 \\
139.0021\end{array}$ & Tectorigenin & 15.18 & 46.57 \\
\hline p77 & 24.77 & {$[\mathrm{M}-\mathrm{H}]^{-}$} & 301.0722 & 301.0718 & 4.701 & $\mathrm{C}_{16} \mathrm{H}_{14} \mathrm{O}_{6}$ & $\begin{array}{l}301.0717 \\
284.0328 \\
257.0478 \\
\text { 164.0106; } \\
\text { 151.0026; } \\
\text { 123.0440; } \\
80.9637\end{array}$ & Hesperetin & 0.06 & 29.45 \\
\hline p78 & 24.87 & {$[\mathrm{M}-\mathrm{H}]^{-}$} & 475.0887 & 475.0882 & 3.258 & $\mathrm{C}_{22} \mathrm{H}_{20} \mathrm{O}_{12}$ & $\begin{array}{l}\text { 475.0868; } \\
\text { 433.8798; } \\
299.0565 ; \\
284.0330 ; \\
153.0180 \\
113.0231\end{array}$ & $\begin{array}{l}\text { 5,7,8-Trihydroxy-6-methoxy flavone-7-0- } \\
\text { glucuronide }\end{array}$ & 86.81 & 29.17 \\
\hline p79* & 24.89 & $\begin{array}{l}{[\mathrm{M}+} \\
\mathrm{FA}-\mathrm{H}]^{-}\end{array}$ & 491.1202 & 491.1195 & 3.741 & $\mathrm{C}_{22} \mathrm{H}_{22} \mathrm{O}_{10}$ & $\begin{array}{l}351.9693 ; \\
283.0614 ; \\
268.0377 ; \\
130.2955 ; \\
91.9576\end{array}$ & Tilianin & 0.76 & 237.40 \\
\hline p80* & 25.06 & {$[\mathrm{M}-\mathrm{H}]^{-}$} & 459.0939 & 459.0933 & 3.795 & $\mathrm{C}_{22} \mathrm{H}_{20} \mathrm{O}_{11}$ & $\begin{array}{l}283.0610 ; \\
268.0379 \\
175.0235 \\
129.0180 \\
113.0230\end{array}$ & Wogonoside & 114.52 & 610.36 \\
\hline p81 & 25.43 & $\begin{array}{l}{[\mathrm{M}+} \\
\mathrm{Na}]^{+}\end{array}$ & 231.0627 & 231.0649 & -0.217 & $\mathrm{C}_{11} \mathrm{H}_{12} \mathrm{O}_{4}$ & $\begin{array}{l}231.1370 ; \\
220.4256 ; \\
185.1321 ; \\
173.0960 ; \\
145.0645 ; \\
131.0851 ; \\
105.0696\end{array}$ & Ethyl caffeate & 0.38 & 26.29 \\
\hline p82* & 25.54 & {$[\mathrm{M}-\mathrm{H}]^{-}$} & 445.0782 & 445.0776 & 3.645 & $\mathrm{C}_{21} \mathrm{H}_{18} \mathrm{O}_{11}$ & $\begin{array}{l}445.0753 \\
283.0615 \\
269.0458 \\
225.0559 \\
175.0245 \\
113.0231\end{array}$ & Baicalein $6-\beta$-D-glucuronide & 142.76 & 25.71 \\
\hline p83* & 25.63 & {$[\mathrm{M}-\mathrm{H}]^{-}$} & 359.0781 & 359.0772 & 5.336 & $\mathrm{C}_{18} \mathrm{H}_{16} \mathrm{O}_{8}$ & $\begin{array}{l}359.0777 \\
344.0541 \\
329.0308 \\
314.0082 \\
285.0403 \\
127.6942\end{array}$ & Rosmarinic acid & 0.68 & 82.92 \\
\hline
\end{tabular}




\begin{tabular}{|c|c|c|c|c|c|c|c|c|c|c|}
\hline \multirow[t]{2}{*}{ NO. } & \multirow{2}{*}{$\begin{array}{l}\mathrm{RT} \\
\text { /min }\end{array}$} & \multirow{2}{*}{$\begin{array}{l}\text { Ion } \\
\text { model }\end{array}$} & \multirow{2}{*}{$\begin{array}{l}\text { Measured } \\
\text { mass /Da }\end{array}$} & \multirow{2}{*}{$\begin{array}{l}\text { Calculated } \\
\text { mass /Da }\end{array}$} & \multirow{2}{*}{$\begin{array}{l}\text { Error } \\
\text { /ppm }\end{array}$} & \multirow{2}{*}{$\begin{array}{l}\text { Molecular } \\
\text { formula }\end{array}$} & \multirow{2}{*}{$\begin{array}{l}\text { Fragment } \\
\text { ions }\end{array}$} & \multirow[t]{2}{*}{ Identification } & \multicolumn{2}{|c|}{ Peak Area $\left({ }^{*} 10^{6}\right)$} \\
\hline & & & & & & & & & Serum & Lung \\
\hline p84 & 25.73 & {$[\mathrm{M}-\mathrm{H}]^{-}$} & 269.0458 & 269.0455 & 4.944 & $\mathrm{C}_{15} \mathrm{H}_{10} \mathrm{O}_{5}$ & $\begin{array}{l}\text { 269.0457; } \\
\text { 239.0349; } \\
\text { 197.0600; } \\
\text { 164.9820; } \\
\text { 136.9867; } \\
85.0282\end{array}$ & Norwogonin & 15.91 & 74.86 \\
\hline p85 & 25.93 & {$[\mathrm{M}-\mathrm{H}]^{-}$} & 489.1046 & 489.1039 & 3.675 & $\mathrm{C}_{23} \mathrm{H}_{22} \mathrm{O}_{12}$ & $\begin{array}{l}\text { 489.2673; } \\
453.2310 \\
313.0721 \\
298.0486 \\
283.0254 \\
211.0406 \\
113.0232\end{array}$ & $\begin{array}{l}\text { 5,7-Dihydroxy-8,2'-dimethoxyflavone7- } \\
0-\beta \text {-D-glucuronide }\end{array}$ & 1.83 & 18.42 \\
\hline p86* & 26.11 & {$[\mathrm{M}-\mathrm{H}]^{-}$} & 255.0665 & 255.0663 & 5.311 & $\mathrm{C}_{15} \mathrm{H}_{12} \mathrm{O}_{4}$ & $\begin{array}{l}255.0663 \\
237.1504 \\
221.0815 \\
171.1495 \\
153.0182 \\
135.0182 \\
119.0489\end{array}$ & Isoliquiritigenin & 0.57 & 17.94 \\
\hline p87 & 27.73 & $\begin{array}{l}{[\mathrm{M}+} \\
\mathrm{H}]^{+}\end{array}$ & 387.1073 & 387.1074 & -0.449 & $\mathrm{C}_{20} \mathrm{H}_{18} \mathrm{O}_{8}$ & $\begin{array}{l}387.1065 ; \\
372.0831 \\
357.0619 \\
341.0647 \\
326.0788\end{array}$ & Irisflorentin & 2.18 & 108.69 \\
\hline p88 & 28.52 & {$[\mathrm{M}-\mathrm{H}]^{-}$} & 283.0615 & 283.0612 & 5.071 & $\mathrm{C}_{16} \mathrm{H}_{12} \mathrm{O}_{5}$ & $\begin{array}{l}\text { 283.0613; } \\
268.0380 ; \\
255.0301 ; \\
215.1290 ; \\
\text { 164.01117; } \\
\text { 133.0653; } \\
87.0084\end{array}$ & Wogonin & 6.53 & 200.24 \\
\hline p89 & 28.77 & {$[\mathrm{M}-\mathrm{H}]^{-}$} & 313.0722 & 313.0718 & 4.809 & $\mathrm{C}_{17} \mathrm{H}_{14} \mathrm{O}_{6}$ & $\begin{array}{l}313.0720 \\
298.0485 \\
283.0250 \\
253.0506 \\
211.0397 \\
173.0594 \\
131.0705\end{array}$ & 3',4'-Dihydroxy-7,5'-dime thoxyflavone & 1.11 & 4.21 \\
\hline p90 & 29.06 & {$[\mathrm{M}-\mathrm{H}]^{-}$} & 983.4514 & 983.4493 & -5.459 & $\mathrm{C}_{48} \mathrm{H}_{72} \mathrm{O}_{21}$ & $\begin{array}{l}\text { 983.4497; } \\
\text { 821.3976; } \\
\text { 803.3886; } \\
645.3657 ; \\
351.0573 \\
289.0569 \\
193.0347\end{array}$ & Licorice saponin A3 & 1.02 & 2.54 \\
\hline p91 & 29.31 & $\begin{array}{l}{[\mathrm{M}+} \\
\mathrm{H}]^{+}\end{array}$ & 403.1387 & 403.1387 & -0.109 & $\mathrm{C}_{21} \mathrm{H}_{22} \mathrm{O}_{8}$ & $\begin{array}{l}403.1381 ; \\
373.0911 \\
355.0809 \\
211.0220 \\
151.0752 \\
79.7506\end{array}$ & Nobiletin & 1.56 & 148.43 \\
\hline p92 & 29.37 & $\begin{array}{l}{[\mathrm{M}+} \\
\mathrm{FA}-\mathrm{H}]^{-}\end{array}$ & 549.3441 & 549.3433 & 3.395 & $\mathrm{C}_{30} \mathrm{H}_{48} \mathrm{O}_{6}$ & $\begin{array}{l}549.3024 \\
485.3278 \\
467.3163 \\
367.2645 \\
249.1490 \\
191.1072 \\
139.0753\end{array}$ & 16-Oxo-alisol A & 0.17 & 8.36 \\
\hline p93 & 29.42 & $\begin{array}{l}{[\mathrm{M}+} \\
\mathrm{H}]^{+}\end{array}$ & 285.0757 & 285.0758 & -0.351 & $\mathrm{C}_{16} \mathrm{H}_{12} \mathrm{O}_{5}$ & $\begin{array}{l}285.0751 \\
270.0519 \\
242.0569 \\
153.0182 \\
133.0648 \\
62.4481\end{array}$ & Oroxylin A & 7.78 & 130.51 \\
\hline p94 & 29.53 & {$[\mathrm{M}-\mathrm{H}]^{-}$} & 329.2338 & 329.2333 & 4.706 & $\mathrm{C}_{18} \mathrm{H}_{34} \mathrm{O}_{5}$ & $\begin{array}{l}329.2338 \\
311.2223 \\
229.1443 \\
211.1335 \\
171.1015 \\
127.1015\end{array}$ & Pinellic acid & 2.95 & 74.22 \\
\hline
\end{tabular}




\begin{tabular}{|c|c|c|c|c|c|c|c|c|c|c|}
\hline \multirow[t]{2}{*}{ No. } & \multirow{2}{*}{$\begin{array}{l}\mathrm{RT} \\
\text { /min }\end{array}$} & \multirow{2}{*}{$\begin{array}{l}\text { lon } \\
\text { model }\end{array}$} & \multirow{2}{*}{$\begin{array}{l}\text { Measured } \\
\text { mass /Da }\end{array}$} & \multirow{2}{*}{$\begin{array}{l}\text { Calculated } \\
\text { mass /Da }\end{array}$} & \multirow{2}{*}{$\begin{array}{l}\text { Error } \\
\text { /ppm }\end{array}$} & \multirow{2}{*}{$\begin{array}{l}\text { Molecular } \\
\text { formula }\end{array}$} & \multirow{2}{*}{$\begin{array}{l}\text { Fragment } \\
\text { ions }\end{array}$} & \multirow[t]{2}{*}{ Identification } & \multicolumn{2}{|c|}{ Peak Area $\left({ }^{*} 10^{6}\right)$} \\
\hline & & & & & & & & & Serum & Lung \\
\hline p95 & 29.80 & {$[\mathrm{M}-\mathrm{H}]^{-}$} & 329.2339 & 329.2333 & 5.071 & $\mathrm{C}_{18} \mathrm{H}_{34} \mathrm{O}_{5}$ & $\begin{array}{l}329.2337 \\
311.2232 \\
\text { 293.2121; } \\
\text { 211.1335; } \\
\text { 171.1017; } \\
\text { 139.1116; } \\
99.0801\end{array}$ & Pinellic acid isomer & 2.46 & 35.22 \\
\hline p96 & 30.33 & {$[\mathrm{M}-\mathrm{H}]^{-}$} & 329.2338 & 329.2333 & 4.797 & $\mathrm{C}_{18} \mathrm{H}_{34} \mathrm{O}_{5}$ & $\begin{array}{l}329.2337 \\
311.2227 \\
251.1648 \\
211.1126 \\
\text { 171.1017; } \\
\text { 139.1116; } \\
99.0801\end{array}$ & Pinellic acid isomer & 2.46 & 13.65 \\
\hline p97 & 30.38 & {$[\mathrm{M}-\mathrm{H}]^{-}$} & 353.1033 & 353.1031 & 3.867 & $\mathrm{C}_{20} \mathrm{H}_{18} \mathrm{O}_{6}$ & $\begin{array}{l}353.1033 \\
285.1136 \\
285.1136 \\
254.0584 \\
193.0856 \\
125.0232\end{array}$ & Licoisoflavone A & 0.45 & 19.92 \\
\hline p98 & 30.76 & $\begin{array}{l}{[\mathrm{M}+} \\
\mathrm{H}]^{+}\end{array}$ & 373.1282 & 373.1282 & -0.025 & $\mathrm{C}_{20} \mathrm{H}_{20} \mathrm{O}_{7}$ & $\begin{array}{l}373.1271 ; \\
343.0806 \\
325.0700 \\
297.0748 \\
217.0815 \\
121.0648\end{array}$ & Tangeretin & 3.84 & 101.45 \\
\hline p99 & 30.96 & {$[\mathrm{M}-\mathrm{H}]^{-}$} & 837.3931 & 837.3914 & 3.276 & $\mathrm{C}_{42} \mathrm{H}_{62} \mathrm{O}_{17}$ & $\begin{array}{l}\text { 837.3947; } \\
775.3915 ; \\
\text { 661.3590; } \\
\text { 485.3269; } \\
\text { 351.0573; } \\
\text { 193.0347; } \\
\text { 71.0123 }\end{array}$ & Licorice saponin Q2 & 2.55 & 8.08 \\
\hline p100* & 31.84 & {$[\mathrm{M}-\mathrm{H}]^{-}$} & 821.3980 & 821.3965 & 3.090 & $\mathrm{C}_{42} \mathrm{H}_{62} \mathrm{O}_{16}$ & $\begin{array}{l}\text { 821.3978; } \\
\text { 645.3635; } \\
351.0574 ; \\
193.0347\end{array}$ & Glycyrrhizic acid & 14.12 & 40.48 \\
\hline p101* & 33.42 & {$[\mathrm{M}-\mathrm{H}]$} & 779.4603 & 779.4576 & 3.464 & $\mathrm{C}_{42} \mathrm{H}_{68} \mathrm{O}_{13}$ & $\begin{array}{l}779.4603 \\
\text { 617.4066; } \\
\text { 471.3486; } \\
\text { 179.0548; } \\
\text { 161.0445; } \\
\text { 145.0495; } \\
101.0230\end{array}$ & Saikosaponin A & 0.08 & 14.77 \\
\hline p102 & 38.23 & $\begin{array}{l}{[\mathrm{M}+} \\
\mathrm{H}]^{+}\end{array}$ & 513.3551 & 513.3575 & -4.580 & $\mathrm{C}_{32} \mathrm{H}_{48} \mathrm{O}_{5}$ & $\begin{array}{l}\text { 513.3542; } \\
496.3501 ; \\
446.9593 \\
316.2172 ; \\
255.7422 \\
169.3950\end{array}$ & Alisol 0 & 46.16 & 106.65 \\
\hline p103* & 38.24 & $\begin{array}{l}{[\mathrm{M}+} \\
\mathrm{FA}-\mathrm{H}]^{-}\end{array}$ & 535.3648 & 535.3640 & 3.399 & $\mathrm{C}_{30} \mathrm{H}_{50} \mathrm{O}_{5}$ & $\begin{array}{l}535.3384 \\
475.3167 \\
415.0358 \\
329.1729 \\
299.0611 \\
269.1505 \\
167.0551\end{array}$ & Alisol A & 37.75 & 91.35 \\
\hline p104* & 39.37 & $\begin{array}{l}{[\mathrm{M}+} \\
\mathrm{FA}-\mathrm{H}]^{-}\end{array}$ & 517.3539 & 517.3535 & 3.043 & $\mathrm{C}_{30} \mathrm{H}_{48} \mathrm{O}_{4}$ & $\begin{array}{l}\text { 517.3328; } \\
\text { 311.2019; } \\
\text { 253.1591; } \\
\text { 169.5029; } \\
108.0066\end{array}$ & Alisol B & 31.79 & 17.44 \\
\hline
\end{tabular}

p- is expressed as prototype; ${ }^{*}$-is expressed as prototype and confirmed by reference substance; RT-retention time

Flavonoids were major constituents in several herbs for preparing QFPDD, it is necessary to identify the absorbed flavonoids in serum from QFPDD-treated mice. In this study, a total of 54 flavonoids (as the absorbed prototype constituents) were detected in mice serum. Take compound p87 for example, this peak generated a quasi-molecular ion $[\mathrm{M}+\mathrm{H}]^{+}$at $m / z 387.1073$, along with characteristic fragment ions at $m / z 372.0831\left[\mathrm{M}+\mathrm{H}-\mathrm{CH}_{3}\right]^{+}, 357.0619\left[\mathrm{M}+\mathrm{H}-\mathrm{CH}_{3}-\mathrm{CH}_{3}\right]^{+}$, $341.0747\left[\mathrm{M}+\mathrm{H}-\mathrm{CH}_{3}-\mathrm{CH}_{3} \mathrm{O}\right]^{+}, 326.0788\left[\mathrm{M}+\mathrm{H}-\mathrm{CH}_{3}-\mathrm{CH}_{2} \mathrm{O}_{2}\right]^{+}$, which was highly consistent with the product ions of compound 302 in QFPDD. As a result, compound $\mathbf{p} \mathbf{8 7}$ was identified as the prototype of compound $\mathbf{3 0 2}$ (irisflorentin). Similarly, compound p57 shared analogical elution time and identical ions in 
$\mathrm{MS}^{2}$ spectra with compound 188 in the extract of QFPDD, such as $\mathrm{m} / z 301.07\left[\mathrm{M}-\mathrm{H}-\mathrm{C}_{12} \mathrm{H}_{20} \mathrm{O}_{9}\right]^{-}, 286.05\left[\mathrm{M}-\mathrm{H}-\mathrm{C}_{12} \mathrm{H}_{20} \mathrm{O}_{9}-\mathrm{CH}_{3}\right]^{-}, 257.08\left[\mathrm{M}-\mathrm{H}-\mathrm{C}_{12} \mathrm{H}_{20} \mathrm{O}_{9}-\mathrm{CO}_{2}\right]^{-}$, 242.06[ $\left[\mathrm{M}-\mathrm{H}-\mathrm{C}_{12} \mathrm{H}_{20} \mathrm{O}_{9}-\mathrm{CH}_{3}-\mathrm{CO}_{2}\right]^{-}$. For this reason, compound $\mathbf{p 5 7}$ was then deemed to be hesperidin, i.e., the prototype of compound 188.

Triterpene saponins were another major group of bioactive components in QFPDD, and it was found that the majority of them could be absorbed into serum. For example, compound $\mathbf{p} 103$ gave molecular formula $\mathrm{C}_{30} \mathrm{H}_{50} \mathrm{O}_{5}$ and deprotonated molecule at $\mathrm{m} / \mathrm{z} 535.3648$ [M-H] ${ }^{-}$, along with distinctive fragment ions at $\mathrm{m} / \mathrm{z}$ generated deprotonated molecule $[\mathrm{M}-\mathrm{H}]^{-}$at $\mathrm{m} / \mathrm{z} 475.3167,415.0358,329.1729,299.0611,269.1505,167.0551$, which was in keeping with compound 396 in the extract of QFPDD. Then, compound p103 was regarded as prototype of compound 396 Alisol A.

Similarly, compound p50 (an organic acid) occurring in rat serum showed identical retention times and quasi-molecular ion $\left(\mathrm{m} / \mathrm{z} 515.1202[\mathrm{M}-\mathrm{H}]^{-}\right.$) to compound 158 in QFPDD. Furthermore, they also produced near identical fragment ions in $\mathrm{MS}^{2}$ spectra, e.g., $m / z \mathrm{~m} / z 353.0884\left[\mathrm{M}-\mathrm{H}-\mathrm{C}_{9} \mathrm{H}_{6} \mathrm{O}_{3}\right]^{-}, 191.0554[\mathrm{M}-\mathrm{H}-$ $\left.\mathrm{C}_{9} \mathrm{H}_{6} \mathrm{O}_{3}-\mathrm{C}_{9} \mathrm{H}_{6} \mathrm{O}_{3}\right]^{-}, 179.0337\left[\mathrm{M}-\mathrm{H}-\mathrm{C}_{9} \mathrm{H}_{7} \mathrm{O}_{4}-\mathrm{C}_{9} \mathrm{H}_{7} \mathrm{O}_{4}\right]^{-}, 161.0234\left[\mathrm{M}-\mathrm{H}-\mathrm{C}_{9} \mathrm{H}_{6} \mathrm{O}_{3}-\mathrm{C}_{7} \mathrm{H}_{13} \mathrm{O}_{5}\right]^{-}$. Thus, compound p50 was identified as the prototype of compound 158, namely isochlorogenic acid $\mathrm{A}$.

\subsubsection{Identification of the metabolites in mice serum}

Next, the metabolites in serum from QFPDD-treated mice that are derived from the constituents in QFPDD were tentatively characterized. To discriminate the endogenous metabolites from QFPDD-related metabolites, the TIC chromatograms of QFPDD-treated mice serum were compared with that of the blank serum. After then, the endogenous substances could be excluded, while the exogenous compounds could be identified by comprehensive analysis. By contrasting these compounds with already known compounds in vitro, searching for the same compounds and validating them via comparison of the retention time and the product ions. TraceFinder software incorporated in UHPLC-Q-Exactive-Orbitrap HRMS could process the data acquired, provide key information about group changes from prototypes to metabolites by means of exact molecular weight, and provide elementary compositions thereof. These analyses were beneficial to determine the prototypes and the metabolites in biological samples, and to conjecture potential metabolic pathways. Furthermore, in this study, parts of in vivo metabolites of the major constituents in QFPDD were identified via comparison with the retention times and MS/MS spectra of the in vitro metabolites generated in MLMs.

Taking metabolites M27, M30 and $\mathbf{M} 65$ as the examples, these three compounds gave similar quasi-molecular ion [M-H] ${ }^{-}$at $m / z 431.0983$, as well as characteristic fragment ions at $m / z 255.0665\left[-176 \mathrm{Da}, \mathrm{M}-\mathrm{H}_{-} \mathrm{C}_{6} \mathrm{H}_{8} \mathrm{O}_{6}\right]^{-}, m / z 135.0090\left[\mathrm{M}-\mathrm{H}-\mathrm{C}_{6} \mathrm{H}_{8} \mathrm{O}_{6}-\mathrm{C}_{8} \mathrm{H}_{8} \mathrm{O}\right]^{-}, m / z 119.0501\left[\mathrm{M}-\mathrm{H}-\mathrm{C}_{6} \mathrm{H}_{8} \mathrm{O}_{6}-\mathrm{C}_{7} \mathrm{H}_{4} \mathrm{O}_{3}\right]^{-}$in the $\mathrm{MS}^{2}$ spectrum. The fragment ions of these three metabolites were identical with that of compound $\mathbf{2 0 5}$ (liquiritigenin), a major constituent identified from the extract of QFPDD. In vitro metabolism of liquiritigenin in MLMs incubated with both NADPH and UDPGA, three mono- $O$-glucuronides could be easily detected. The retention times, product ions were consistent with the metabolites M27, M30 and M65. In these cases, these three compounds were identified as liquiritigenin-O-glucuronide. Similarly, the in vivo metabolites $\mathbf{M 3 4}$ and $\mathbf{M 7 0}$ are identified as typical sulfates, both compounds displayed similar quasimolecular ion $[\mathrm{M}-\mathrm{H}]^{-}$at $m / z 335.0231$, as well as characteristic fragment ions at $m / z 255.0665\left[-80 \mathrm{Da}, \mathrm{M}-\mathrm{H}-\mathrm{SO}_{3}\right]^{-}, m / z 135.0090\left[\mathrm{M}^{-} \mathrm{H}-\mathrm{SO}_{3}-\mathrm{C}_{8} \mathrm{H}_{8} \mathrm{O}\right]^{-}, m / z$ $119.0501\left[\mathrm{M}-\mathrm{H}-\mathrm{SO}_{3}-\mathrm{C}_{7} \mathrm{H}_{4} \mathrm{O}_{3}\right]^{-}$in the $\mathrm{MS}^{2}$ spectrum, the fragment ions were identical with that of the authentic standard liquiritigenin. In vitro metabolism demonstrated that following incubation of liquiritigenin in MLMs in the presence of both NADPH and PAPS, two mono- $O$-sulfates could be easily detected. The retention times, product ions were well-matched with the metabolites M34 and M70. Thus, these two peaks were identified as liquiritigenin- 0 -sulfate.

The metabolite $\mathbf{M} 21$ and $\mathbf{M} 25$ were produced from an organic acid. They gave similar quasi-molecular ion [M- $\mathrm{H}^{-}$at $\mathrm{m} / \mathrm{z} 367.1034$, as well as characteristic fragment ions at $m / z 353.0883\left[-15 \mathrm{Da}, \mathrm{M}-\mathrm{H}-\mathrm{CH}_{3}\right]^{-}, m / z 191.0549\left[\mathrm{M}-\mathrm{H}_{-} \mathrm{CH}_{3}-\mathrm{C}_{9} \mathrm{H}_{6} \mathrm{O}_{3}\right]^{-}, m / z 179.0337\left[\mathrm{M}-\mathrm{H}_{-} \mathrm{CH}_{3}-\mathrm{C}_{7} \mathrm{H}_{10} \mathrm{O}_{5}\right]^{-}, m / z 173.0442\left[\mathrm{M}-\mathrm{H}-\mathrm{C}_{9} \mathrm{H}_{6} \mathrm{O}_{3}-\mathrm{H}_{2} \mathrm{O}\right]^{-}$, $\mathrm{m} / \mathrm{z} 161.0228\left[\mathrm{M}-\mathrm{H}_{-} \mathrm{CH}_{3}-\mathrm{C}_{7} \mathrm{H}_{10} \mathrm{O}_{5}-\mathrm{H}_{2} \mathrm{O}\right]^{-}$in the $\mathrm{MS}^{2}$ spectrum, the fragment ions were identical with the compound $\mathbf{5 9}$ (neochlorogenic acid), 80 (chlorogenic acid), 87 (cryptochlorogenic acid) in the extract of QFPDD. In vitro metabolism demonstrated that following incubation of chlorogenic acid in MLMs in the presence of NADPH and SAM, two $O$-methylated metabolites were detected. The retention times, product ions were well-matched with that of the metabolites M21 and M25. Thus, these two metabolites were identified as $O$-methylated chlorogenic acid. Previous study have reported that two $O$-methylated metabolites of chlorogenic acid were found in urine samples after intramuscular administration of chlorogenic acid injection to healthy adults, and the pure $O$-methylated metabolites were synthesized by chlorogenic acid and followed by isolation, purification and identification [22]. According to the chromatographic behavior of them, M21 was tentatively identified as 3'- methyl-chlorogenic acid, and M25 was tentatively identified as 4'- methyl-chlorogenic acid.

Following carefully metabolite identification as mentioned above, a total of 91 metabolites were tentatively identified (Table 3). A variety of metabolic reactions, such as hydroxylation, $O$-methylation, $O$-glucuronidation, $O$-sulfation, demethylation, were involved in the metabolism of the major constituents in QFPDD. Among them, 20 metabolites are fully confirmed by the in vitro metabolites generated in RLM. 
Table 3

Identification of metabolites of QFPDD in rat serum and multiple tissues by UHPLC-Q-Exactive Orbitrap HRMS

\begin{tabular}{|c|c|c|c|c|c|c|c|c|c|c|c|c|}
\hline \multirow[t]{2}{*}{ NO } & \multirow{2}{*}{$\begin{array}{l}\mathrm{RT} \\
/ \mathrm{min}\end{array}$} & \multirow{2}{*}{$\begin{array}{l}\text { lon } \\
\text { model }\end{array}$} & \multirow{2}{*}{$\begin{array}{l}\text { Measured } \\
\text { mass /Da }\end{array}$} & \multirow{2}{*}{$\begin{array}{l}\text { Calculated } \\
\text { mass /Da }\end{array}$} & \multirow{2}{*}{$\begin{array}{l}\text { Error } \\
\text { /ppm }\end{array}$} & \multirow{2}{*}{$\begin{array}{l}\text { Molecular } \\
\text { formula }\end{array}$} & \multirow{2}{*}{$\begin{array}{l}\text { Fragment } \\
\text { ions }\end{array}$} & \multirow[t]{2}{*}{ Identification } & \multicolumn{4}{|c|}{ Peak Area $\left(* 10^{6}\right)$} \\
\hline & & & & & & & & & Serum & Lung & Liver & $\mathrm{He}$ \\
\hline M1 & 4.45 & $\begin{array}{l}{[\mathrm{M}+} \\
\mathrm{H}]^{+}\end{array}$ & 134.0590 & 134.0600 & -7.8 & $\mathrm{C}_{8} \mathrm{H}_{7} \mathrm{NO}$ & $\begin{array}{l}\text { 106.0629; } \\
79.0536\end{array}$ & Mandelonitrile & 83.46 & 43.02 & 96.52 & 70 \\
\hline M2 & 8.16 & {$[\mathrm{M}-\mathrm{H}]^{-}$} & 477.0648 & 477.0674 & -5.4 & $\mathrm{C}_{21} \mathrm{H}_{18} \mathrm{O}_{13}$ & $\begin{array}{l}477.1811 ; \\
343.0462 \\
301.0357 \\
193.0138 \\
151.0025 \\
125.0232\end{array}$ & Quercetin-glucuronide & 0.85 & 0.00 & 0.00 & $0 . c$ \\
\hline M3 & 8.49 & {$[\mathrm{M}-\mathrm{H}]^{-}$} & 371.0970 & 371.0983 & -3.7 & $\mathrm{C}_{16} \mathrm{H}_{20} \mathrm{O}_{10}$ & $\begin{array}{l}\text { 371.0987; } \\
\text { 309.0988; } \\
248.9853 ; \\
223.0609 ; \\
195.0657 ; \\
157.0131 ; \\
113.0231\end{array}$ & $\begin{array}{l}\text { Caffeic acid- } \\
\text { methylation- } \\
\text { glucuronide-reduction }\end{array}$ & 1.18 & 0.00 & 0.25 & $0 . c$ \\
\hline M4\# & 10.37 & {$[\mathrm{M}-\mathrm{H}]^{-}$} & 258.9918 & 258.9918 & 0.0 & $\mathrm{C}_{9} \mathrm{H}_{8} \mathrm{SO}_{7}$ & $\begin{array}{l}\text { 179.0342; } \\
\text { 135.0439; } \\
96.9587 ; \\
79.9558\end{array}$ & Caffeic acid-sulfate & 234.36 & 1.52 & 39.90 & 19 \\
\hline M5 & 11.25 & {$[\mathrm{M}-\mathrm{H}]^{-}$} & 637.1031 & 637.1046 & -2.4 & $\mathrm{C}_{27} \mathrm{H}_{26} \mathrm{O}_{18}$ & 285.0395 & $\begin{array}{l}\text { Scutellarein-di- } \\
\text { glucuronide }\end{array}$ & 0.02 & 0.00 & 0.00 & $0 . c$ \\
\hline M6 & 12.05 & $\begin{array}{l}{[\mathrm{M}+} \\
\mathrm{FA}-\mathrm{H}]^{-}\end{array}$ & 293.1248 & 293.1242 & 5.786 & $\mathrm{C}_{11} \mathrm{H}_{20} \mathrm{O}_{6}$ & $\begin{array}{l}293.1243 \\
232.1332 \\
187.1077 \\
131.0687 \\
119.0332 \\
89.0217\end{array}$ & $\begin{array}{l}\text { Pent-4-enyl-D- } \\
\text { glucopyranoside } \\
\text { isomer }\end{array}$ & 4.73 & 4.53 & 8.86 & 3.1 \\
\hline M7 & 12.68 & {$[\mathrm{M}-\mathrm{H}]^{-}$} & 477.0670 & 477.0674 & -0.8 & $\mathrm{C}_{21} \mathrm{H}_{18} \mathrm{O}_{13}$ & $\begin{array}{l}477.1844 \\
372.8266 \\
301.0358 \\
283.0250 \\
193.0136 \\
151.0026\end{array}$ & Quercetin-glucuronide & 1.64 & 0.00 & 0.00 & $0 . c$ \\
\hline M8 & 13.09 & {$[\mathrm{M}-\mathrm{H}]^{-}$} & 256.9764 & 256.9761 & 1.2 & $\mathrm{C}_{9} \mathrm{H}_{6} \mathrm{SO}_{7}$ & $\begin{array}{l}\text { 239.2304; } \\
\text { 198.4493; } \\
\text { 177.0186; } \\
\text { 159.1671; } \\
133.0649\end{array}$ & $\begin{array}{l}5,7- \\
\text { Dihydroxychromone- } \\
\text { sulfate }\end{array}$ & 18.35 & 1.95 & 1.41 & 2.c \\
\hline M9 & 14.01 & $\begin{array}{l}{[\mathrm{M}+} \\
\mathrm{FA}-\mathrm{H}]^{-}\end{array}$ & 293.1247 & 293.1242 & 5.445 & $\mathrm{C}_{11} \mathrm{H}_{20} \mathrm{O}_{6}$ & $\begin{array}{l}293.1245 \\
232.1336 \\
187.1082 \\
131.0701 \\
119.0337 \\
89.0229\end{array}$ & $\begin{array}{l}\text { Pent-4-enyl-D- } \\
\text { glucopyranoside isomer }\end{array}$ & 3.14 & 3.79 & 20.25 & $0 . c$ \\
\hline M10 & 14.40 & {$[\mathrm{M}-\mathrm{H}]^{-}$} & 294.0989 & 294.0983 & 5.564 & $\mathrm{C}_{14} \mathrm{H}_{17} \mathrm{NO}_{6}$ & $\begin{array}{l}251.0562 ; \\
161.0446 \\
121.0282 \\
113.0231 \\
85.0280 \\
71.0123\end{array}$ & Prunasin isomer & 8.31 & 1.17 & 8.43 & 8.4 \\
\hline M11 & 14.53 & {$[\mathrm{M}-\mathrm{H}]^{-}$} & 369.0820 & 369.0827 & -2.0 & $\mathrm{C}_{16} \mathrm{H}_{18} \mathrm{O}_{10}$ & $\begin{array}{l}\text { 193.0543; } \\
164.9292 \\
120.9383\end{array}$ & $\begin{array}{l}\text { Chlorogenic acid- } \\
\text { methylation }\end{array}$ & 0.60 & 0.00 & 1.65 & $0 . c$ \\
\hline M12 & 14.59 & $\begin{array}{l}{[\mathrm{M}+} \\
\mathrm{FA}-\mathrm{H}]^{-}\end{array}$ & 293.1247 & 293.1242 & 5.581 & $\mathrm{C}_{11} \mathrm{H}_{20} \mathrm{O}_{6}$ & $\begin{array}{l}293.1244 ; \\
247.0244 ; \\
213.1745 ; \\
173.0812 ; \\
131.0701 ; \\
119.0336 ; \\
89.0229\end{array}$ & $\begin{array}{l}\text { Pent-4-enyl-D- } \\
\text { glucopyranoside isomer }\end{array}$ & 2.32 & 0.68 & 0.80 & $0 . c$ \\
\hline M13 & 14.97 & {$[\mathrm{M}-\mathrm{H}]^{-}$} & 593.1498 & 593.1512 & -2.4 & $\mathrm{C}_{27} \mathrm{H}_{30} \mathrm{O}_{15}$ & $\begin{array}{l}593.1498 \\
417.1192 \\
369.0989 \\
255.0665 \\
175.0240 \\
135.0075\end{array}$ & Liquiritin-glucuronide & 3.71 & 0.17 & 0.38 & $0 . c$ \\
\hline M14 & 15.38 & {$[\mathrm{M}-\mathrm{H}]^{-}$} & 167.0342 & 167.0350 & 1.585 & $\mathrm{C}_{8} \mathrm{H}_{8} \mathrm{O}_{4}$ & 167.0341 & Vanillic acid isomer & 0.55 & 5.52 & 1.18 & $0 . c$ \\
\hline
\end{tabular}




\begin{tabular}{|c|c|c|c|c|c|c|c|c|c|c|c|c|}
\hline \multirow[t]{2}{*}{ NO } & \multirow{2}{*}{$\begin{array}{l}\mathrm{RT} \\
/ \mathrm{min}\end{array}$} & \multirow{2}{*}{$\begin{array}{l}\text { lon } \\
\text { model }\end{array}$} & \multirow{2}{*}{$\begin{array}{l}\text { Measured } \\
\text { mass /Da }\end{array}$} & \multirow{2}{*}{$\begin{array}{l}\text { Calculated } \\
\text { mass / } \mathrm{Da}\end{array}$} & \multirow{2}{*}{$\begin{array}{l}\text { Error } \\
\text { /ppm }\end{array}$} & \multirow{2}{*}{$\begin{array}{l}\text { Molecular } \\
\text { formula }\end{array}$} & \multirow{2}{*}{$\begin{array}{l}\text { Fragment } \\
\text { ions }\end{array}$} & \multirow[t]{2}{*}{ Identification } & \multicolumn{4}{|c|}{ Peak Area $\left(* 10^{6}\right)$} \\
\hline & & & & & & & & & Serum & Lung & Liver & $\mathrm{He}$ \\
\hline M15 & 15.41 & {$[\mathrm{M}-\mathrm{H}]^{-}$} & 327.1090 & 327.1085 & 4.787 & $\mathrm{C}_{15} \mathrm{H}_{20} \mathrm{O}_{8}$ & $\begin{array}{l}\text { 327.1416; } \\
\text { 266.1181; } \\
\text { 211.1447; } \\
\text { 164.0706; } \\
\text { 147.0440; } \\
\text { 113.0230; } \\
89.0229\end{array}$ & $\begin{array}{l}\text { Dihydrocinnacasside } \\
\text { isomer }\end{array}$ & 3.76 & 3.71 & 4.81 & $0 . C$ \\
\hline M16 & 15.82 & {$[\mathrm{M}-\mathrm{H}]^{-}$} & 167.0342 & 167.0350 & 1.645 & $\mathrm{C}_{8} \mathrm{H}_{8} \mathrm{O}_{4}$ & 167.0342 & Vanillic acid isomer & 3.04 & 0.00 & 0.78 & $0 . c$ \\
\hline M17 & 15.91 & {$[\mathrm{M}-\mathrm{H}]^{-}$} & 637.1017 & 637.1046 & -4.6 & $\mathrm{C}_{27} \mathrm{H}_{26} \mathrm{O}_{18}$ & 637.1015 & $\begin{array}{l}\text { Scutellarein-di- } \\
\text { glucuronide }\end{array}$ & 0.13 & 0.00 & 0.00 & $0 . c$ \\
\hline M18 & 16.05 & {$[\mathrm{M}-\mathrm{H}]^{-}$} & 511.0535 & 511.0552 & -3.3 & $\mathrm{C}_{21} \mathrm{H}_{20} \mathrm{O}_{13} \mathrm{~S}$ & $\begin{array}{l}431.0993 \\
335.0238 \\
255.0665 \\
217.0175 \\
175.0241 \\
135.0077\end{array}$ & Liquiritin-sulfate & 0.65 & 0.00 & 3.29 & $0 . c$ \\
\hline M19 & 16.27 & {$[\mathrm{M}-\mathrm{H}]^{-}$} & 371.0960 & 371.0983 & -6.4 & $\mathrm{C}_{16} \mathrm{H}_{20} \mathrm{O}_{10}$ & $\begin{array}{l}\text { 371.0990; } \\
293.0681 ; \\
243.1061 ; \\
195.1021 ; \\
180.0782 ; \\
129.0182 ; \\
113.0232\end{array}$ & $\begin{array}{l}\text { Caffeic acid- } \\
\text { methylation- } \\
\text { glucuronide-reduction }\end{array}$ & 3.19 & 9.47 & 15.90 & $1 . \mathrm{C}$ \\
\hline M20 & 16.28 & {$[\mathrm{M}-\mathrm{H}]^{-}$} & 497.0743 & 497.0759 & -3.2 & $\mathrm{C}_{21} \mathrm{H}_{22} \mathrm{O}_{12} \mathrm{~S}$ & $\begin{array}{l}\text { 497.0767; } \\
417.1199 ; \\
323.0241 ; \\
255.0665 ; \\
243.0664 ;\end{array}$ & Liquiritin-sulfate & 0.07 & 0.00 & 1.06 & $0 . c$ \\
\hline M21\# & 16.48 & {$[\mathrm{M}-\mathrm{H}]^{-}$} & 367.1039 & 367.1034 & 1.2 & $\mathrm{C}_{17} \mathrm{H}_{20} \mathrm{O}_{9}$ & $\begin{array}{l}367.1052 \\
353.0871 \\
191.0543 \\
179.0315 \\
173.0444 \\
135.0424\end{array}$ & $\begin{array}{l}\text { 3'- Methyl-chlorogenic } \\
\text { acid }\end{array}$ & 4.12 & 36.78 & 54.07 & $9 . \mathrm{C}$ \\
\hline M22 & 16.52 & {$[\mathrm{M}-\mathrm{H}]^{-}$} & 137.0233 & 137.0244 & -0.369 & $\mathrm{C}_{7} \mathrm{H}_{6} \mathrm{O}_{3}$ & $\begin{array}{l}\text { 137.0231; } \\
93.0331 ; \\
84.8779 \\
70.7439\end{array}$ & $\begin{array}{l}\text { 3,4- } \\
\text { Dihydroxybenzaldehyde } \\
\text { isomer }\end{array}$ & 0.23 & 0.96 & 0.78 & $0 . c$ \\
\hline M23 & 16.85 & {$[\mathrm{M}-\mathrm{H}]^{-}$} & 379.0108 & 379.0129 & -5.6 & $\mathrm{C}_{16} \mathrm{H}_{12} \mathrm{O}_{9} \mathrm{~S}$ & $\begin{array}{l}299.0566 ; \\
284.0330 ; \\
216.1235 ; \\
203.1289 ; \\
169.0320\end{array}$ & $\begin{array}{l}\text { Wogonin-sulfate- } \\
\text { hydroxylation }\end{array}$ & 0.23 & 0.00 & 0.00 & $0 . c$ \\
\hline M24 & 16.97 & {$[\mathrm{M}-\mathrm{H}]^{-}$} & 629.1170 & 629.1182 & -1.9 & $\mathrm{C}_{26} \mathrm{H}_{30} \mathrm{O}_{16} \mathrm{~S}$ & $\begin{array}{l}\text { 629.1162; } \\
255.0603\end{array}$ & $\begin{array}{l}\text { Liquiritin apioside- } \\
\text { sulfate }\end{array}$ & 0.03 & 0.00 & 0.82 & $0 . C$ \\
\hline M25\# & 17.41 & {$[\mathrm{M}-\mathrm{H}]^{-}$} & 367.1039 & 367.1034 & 1.2 & $\mathrm{C}_{17} \mathrm{H}_{20} \mathrm{O}_{9}$ & $\begin{array}{l}367.1043 ; \\
353.0889 \\
191.0537 \\
179.0321 \\
173.0451 \\
135.0435\end{array}$ & $\begin{array}{l}\text { 4'- Methyl-chlorogenic } \\
\text { acid }\end{array}$ & 1.16 & 20.25 & 6.17 & $4 . \varepsilon$ \\
\hline M26 & 17.43 & {$[\mathrm{M}-\mathrm{H}]^{-}$} & 369.0801 & 369.0827 & -7.1 & $\mathrm{C}_{16} \mathrm{H}_{18} \mathrm{O}_{10}$ & $\begin{array}{l}\text { 193.0467; } \\
120.9373\end{array}$ & $\begin{array}{l}\text { Chlorogenic acid- } \\
\text { methylation- } \\
\text { glucuronide }\end{array}$ & 3.11 & 1.32 & 1.05 & $0 . C$ \\
\hline M27\# & 17.79 & {$[\mathrm{M}-\mathrm{H}]^{-}$} & 431.0995 & 431.0983 & 2.8 & $\mathrm{C}_{21} \mathrm{H}_{20} \mathrm{O}_{10}$ & $\begin{array}{l}\text { 255.0700; } \\
\text { 135.0110; } \\
119.0506\end{array}$ & $\begin{array}{l}\text { Liquiritigenin- } \\
\text { glucuronide }\end{array}$ & 51.44 & 4.01 & 33.19 & 7.2 \\
\hline M28 & 17.87 & {$[\mathrm{M}-\mathrm{H}]^{-}$} & 637.1033 & 637.1046 & -2.0 & $\mathrm{C}_{27} \mathrm{H}_{26} \mathrm{O}_{18}$ & $\begin{array}{l}\text { 285.0425; } \\
113.0312\end{array}$ & $\begin{array}{l}\text { Scutellarein-di- } \\
\text { glucuronide }\end{array}$ & 0.25 & 0.00 & 0.00 & $0 . c$ \\
\hline M29 & 18.21 & {$[\mathrm{M}-\mathrm{H}]^{-}$} & 557.0587 & 557.0606 & -3.4 & $\mathrm{C}_{22} \mathrm{H}_{22} \mathrm{O}_{15} \mathrm{~S}$ & $\begin{array}{l}557.0699 \\
477.1009 \\
301.0731 \\
284.9333 \\
216.9426 \\
148.9576\end{array}$ & $\begin{array}{l}\text { Hesperetin-glucuronide- } \\
\text { sulfate }\end{array}$ & 3.20 & 0.00 & 3.42 & $0 . C$ \\
\hline M30\# & 18.53 & {$[\mathrm{M}-\mathrm{H}]^{-}$} & 431.0968 & 431.0983 & -3.5 & $\mathrm{C}_{21} \mathrm{H}_{20} \mathrm{O}_{10}$ & $\begin{array}{l}431.0992 \\
255.0700 \\
135.0118 \\
119.0515\end{array}$ & $\begin{array}{l}\text { Liquiritigenin- } \\
\text { glucuronide }\end{array}$ & 15.45 & 3.21 & 15.38 & 1.2 \\
\hline
\end{tabular}




\begin{tabular}{|c|c|c|c|c|c|c|c|c|c|c|c|c|}
\hline \multirow[t]{2}{*}{ NO } & \multirow{2}{*}{$\begin{array}{l}\mathrm{RT} \\
\text { /min }\end{array}$} & \multirow{2}{*}{$\begin{array}{l}\text { lon } \\
\text { model }\end{array}$} & \multirow{2}{*}{$\begin{array}{l}\text { Measured } \\
\text { mass /Da }\end{array}$} & \multirow{2}{*}{$\begin{array}{l}\text { Calculated } \\
\text { mass / } \mathrm{Da}\end{array}$} & \multirow{2}{*}{$\begin{array}{l}\text { Error } \\
\text { /ppm }\end{array}$} & \multirow{2}{*}{$\begin{array}{l}\text { Molecular } \\
\text { formula }\end{array}$} & \multirow{2}{*}{$\begin{array}{l}\text { Fragment } \\
\text { ions }\end{array}$} & \multirow[t]{2}{*}{ Identification } & \multicolumn{4}{|c|}{ Peak Area $\left({ }^{*} 10^{6}\right)$} \\
\hline & & & & & & & & & Serum & Lung & Liver & $\mathrm{He}$ \\
\hline M31 & 18.94 & {$[\mathrm{M}-\mathrm{H}]^{-}$} & 653.1336 & 653.1359 & -3.5 & $\mathrm{C}_{28} \mathrm{H}_{30} \mathrm{O}_{18}$ & $\begin{array}{l}\text { 477.1069; } \\
415.1058 \\
301.0727 \\
175.0241 \\
113.0231\end{array}$ & $\begin{array}{l}\text { Hesperetin-O- } \\
\text { diglucuronides }\end{array}$ & 0.53 & 0.00 & 0.00 & $0 . C$ \\
\hline M32 & 19.06 & {$[\mathrm{M}-\mathrm{H}]^{-}$} & 785.2153 & 785.2146 & 0.9 & $\mathrm{C}_{34} \mathrm{H}_{42} \mathrm{O}_{21}$ & $\begin{array}{l}\text { 785.2206; } \\
\text { 707.3138; } \\
\text { 609.1837; } \\
\text { 489.1429; } \\
\text { 415.1046; } \\
343.0828 \\
301.0722\end{array}$ & $\begin{array}{l}\text { Hesperidin-glucuronide } \\
\text { or neohesperidin- } \\
\text { glucuronide }\end{array}$ & 0.45 & 0.00 & 0.24 & $0 . C$ \\
\hline M33 & 19.2 & {$[\mathrm{M}-\mathrm{H}]^{-}$} & 333.0057 & 333.0074 & -5.2 & $\mathrm{C}_{15} \mathrm{H}_{10} \mathrm{O}_{7} \mathrm{~S}$ & $\begin{array}{l}333.0082 \\
253.0508 \\
203.1109 \\
135.0076 \\
91.0180\end{array}$ & Daidzein-sulfate & 25.28 & 3.98 & 17.62 & $5 . C$ \\
\hline M34\# & 19.24 & {$[\mathrm{M}-\mathrm{H}]^{-}$} & 335.0215 & 335.0231 & -4.8 & $\mathrm{C}_{15} \mathrm{H}_{12} \mathrm{O}_{7} \mathrm{~S}$ & $\begin{array}{l}255.0699 \\
135.0102 \\
119.0498\end{array}$ & Liquiritigenin-sulfate & 9.25 & 6.15 & 0.00 & $2 . c$ \\
\hline M35 & 19.36 & {$[\mathrm{M}-\mathrm{H}]^{-}$} & 475.0858 & 475.0882 & -5.1 & $\mathrm{C}_{22} \mathrm{H}_{20} \mathrm{O}_{12}$ & $\begin{array}{l}475.1029 \\
299.0536 \\
256.0377 \\
211.0419 \\
133.0297\end{array}$ & $\begin{array}{l}\text { Trihydroxy- } \\
\text { methoxyflavone- } \\
\text { glucuronide }\end{array}$ & 33.79 & 24.02 & 54.73 & 9.6 \\
\hline M36 & 19.37 & $\begin{array}{l}{[\mathrm{M}+} \\
\mathrm{H}]^{+}\end{array}$ & 477.1012 & 477.1028 & -3.3 & $\mathrm{C}_{22} \mathrm{H}_{20} \mathrm{O}_{12}$ & $\begin{array}{l}301.1382 \\
286.0488 \\
168.0059\end{array}$ & $\begin{array}{l}\text { Wogonin-hydroxylation- } \\
\text { glucuronide }\end{array}$ & 22.12 & 15.13 & 52.41 & 5.5 \\
\hline M37 & 19.44 & {$[\mathrm{M}-\mathrm{H}]^{-}$} & 651.1204 & 651.1203 & 0.2 & $\mathrm{C}_{28} \mathrm{H}_{28} \mathrm{O}_{18}$ & $\begin{array}{l}\text { 599.3586; } \\
\text { 475.0865; } \\
299.0565 ; \\
284.0330 \\
175.0241\end{array}$ & $\begin{array}{l}\text { Trihydroxy- } \\
\text { methoxyflavone-di- } \\
\text { glucuronide }\end{array}$ & 4.73 & 0.62 & 0.36 & $0 . c$ \\
\hline M38 & 19.5 & {$[\mathrm{M}-\mathrm{H}]^{-}$} & 785.2146 & 785.2146 & 0.0 & $\mathrm{C}_{34} \mathrm{H}_{42} \mathrm{O}_{21}$ & $\begin{array}{l}\text { 785.2145; } \\
\text { 609.1829; } \\
\text { 489.1393; } \\
\text { 415.1045; } \\
301.0721 \\
284.0343 \\
\text { 175.0241; }\end{array}$ & $\begin{array}{l}\text { Hesperidin-glucuronide } \\
\text { or neohesperidin- } \\
\text { glucuronide }\end{array}$ & 0.34 & 0.00 & 0.00 & $0 . C$ \\
\hline M39 & 19.58 & {$[\mathrm{M}-\mathrm{H}]^{-}$} & 621.1097 & 621.1097 & 0.0 & $\mathrm{C}_{27} \mathrm{H}_{26} \mathrm{O}_{17}$ & $\begin{array}{l}549.3765 \\
445.0777 \\
311.0574 \\
269.0459 \\
193.0349 \\
113.0231\end{array}$ & Baicalein-di-glucuronide & 2.08 & 0.18 & 0.00 & $0 . C$ \\
\hline M40 & 19.6 & {$[\mathrm{M}-\mathrm{H}]^{-}$} & 637.1021 & 637.1046 & -3.9 & $\mathrm{C}_{27} \mathrm{H}_{26} \mathrm{O}_{18}$ & $\begin{array}{l}637.1035 \\
461.0659 \\
285.0406\end{array}$ & $\begin{array}{l}\text { Scutellarein-di- } \\
\text { glucuronide }\end{array}$ & 0.36 & 0.00 & 0.00 & $0 . C$ \\
\hline M41 & 19.63 & {$[\mathrm{M}-\mathrm{H}]^{-}$} & 333.0057 & 333.0074 & -5.2 & $\mathrm{C}_{15} \mathrm{H}_{10} \mathrm{O}_{7} \mathrm{~S}$ & $\begin{array}{l}333.0080 \\
270.1216 \\
253.0508 \\
205.0901 \\
171.1019\end{array}$ & Daidzein-sulfate & 25.28 & 1.08 & 17.59 & $0 . c$ \\
\hline M42 & 19.81 & {$[\mathrm{M}-\mathrm{H}]^{-}$} & 525.0313 & 525.0344 & -6.0 & $\mathrm{C}_{21} \mathrm{H}_{18} \mathrm{O}_{14} \mathrm{~S}$ & $\begin{array}{l}525.0321 \\
445.0763 \\
349.0031 \\
269.0460 \\
175.0241\end{array}$ & Baicalin-sulfate & 8.52 & 0.00 & 11.93 & $0 . c$ \\
\hline M43\# & 19.88 & {$[\mathrm{M}-\mathrm{H}]^{-}$} & 477.1003 & 477.1038 & -7.3 & $\mathrm{C}_{22} \mathrm{H}_{22} \mathrm{O}_{12}$ & $\begin{array}{l}301.0721 \\
286.0483 \\
242.0571 \\
151.0239\end{array}$ & Hesperetin-glucuronide & 0.39 & 0.19 & 2.02 & $0 . c$ \\
\hline M44\# & 19.93 & {$[\mathrm{M}-\mathrm{H}]^{-}$} & 477.0657 & 477.0674 & -3.6 & $\mathrm{C}_{21} \mathrm{H}_{18} \mathrm{O}_{13}$ & $\begin{array}{l}475.2026 \\
301.0720 \\
283.0614 \\
269.0459 \\
204.0074 \\
161.0234\end{array}$ & Quercetin-glucuronide & 3.31 & 0.00 & 0.00 & $0 . C$ \\
\hline
\end{tabular}




\begin{tabular}{|c|c|c|c|c|c|c|c|c|c|c|c|c|}
\hline \multirow[t]{2}{*}{ No } & \multirow{2}{*}{$\begin{array}{l}\mathrm{RT} \\
\text { /min }\end{array}$} & \multirow{2}{*}{$\begin{array}{l}\text { lon } \\
\text { model }\end{array}$} & \multirow{2}{*}{$\begin{array}{l}\text { Measured } \\
\text { mass /Da }\end{array}$} & \multirow{2}{*}{$\begin{array}{l}\text { Calculated } \\
\text { mass /Da }\end{array}$} & \multirow{2}{*}{$\begin{array}{l}\text { Error } \\
\text { /ppm }\end{array}$} & \multirow{2}{*}{$\begin{array}{l}\text { Molecular } \\
\text { formula }\end{array}$} & \multirow{2}{*}{$\begin{array}{l}\text { Fragment } \\
\text { ions }\end{array}$} & \multirow[t]{2}{*}{ Identification } & \multicolumn{4}{|c|}{ Peak Area $\left(* 10^{6}\right)$} \\
\hline & & & & & & & & & Serum & Lung & Liver & $\mathrm{He}$ \\
\hline M45 & 20.06 & {$[\mathrm{M}-\mathrm{H}]^{-}$} & 651.1191 & 651.1203 & -1.8 & $\mathrm{C}_{28} \mathrm{H}_{28} \mathrm{O}_{18}$ & $\begin{array}{l}475.0876 ; \\
413.0883 \\
299.0565 ; \\
284.0330 \\
175.0240\end{array}$ & $\begin{array}{l}\text { Trihydroxy- } \\
\text { methoxyflavone-di- } \\
\text { glucuronide }\end{array}$ & 0.28 & 0.00 & 0.00 & $0 . c$ \\
\hline M46 & 20.28 & {$[\mathrm{M}-\mathrm{H}]^{-}$} & 579.1727 & 579.1719 & 3.277 & $\mathrm{C}_{27} \mathrm{H}_{32} \mathrm{O}_{14}$ & $\begin{array}{l}\text { 417.1561; } \\
\text { 295.0616; } \\
\text { 271.0614; } \\
\text { 181.0498; } \\
\text { 151.0026; } \\
93.0332\end{array}$ & $\begin{array}{l}\text { Liquiritigenin-7,4'- } \\
\text { diglucoside isomer }\end{array}$ & 1.87 & 211.94 & 404.51 & $0 . c$ \\
\hline M47 & 20.77 & {$[\mathrm{M}-\mathrm{H}]^{-}$} & 367.0109 & 367.0129 & -5.5 & $\mathrm{C}_{15} \mathrm{H}_{12} \mathrm{O}_{9} \mathrm{~S}$ & $\begin{array}{l}367.0128 ; \\
287.0570 ; \\
269.0465 ; \\
193.0859 ; \\
151.0027\end{array}$ & $\begin{array}{l}\text { Hesperetin- } \\
\text { demethylation-sulfate }\end{array}$ & 0.48 & 0.21 & 4.16 & $0 . c$ \\
\hline M48 & 20.86 & $\begin{array}{l}{[\mathrm{M}+} \\
\mathrm{H}]^{+}\end{array}$ & 375.1033 & 375.1074 & -11.0 & $\mathrm{C}_{19} \mathrm{H}_{18} \mathrm{O}_{8}$ & $\begin{array}{l}375.1071 ; \\
360.0836 \\
345.0601 \\
329.0645 ; \\
163.0752\end{array}$ & $\begin{array}{l}\text { Nobiletin-di- } \\
\text { demethylation }\end{array}$ & 1.60 & 0.00 & 0.82 & $0 . c$ \\
\hline M49\# & 21.15 & {$[\mathrm{M}-\mathrm{H}]^{-}$} & 477.1007 & 477.1038 & -6.5 & $\mathrm{C}_{22} \mathrm{H}_{22} \mathrm{O}_{12}$ & $\begin{array}{l}301.0722 ; \\
286.0484 ; \\
242.0589 \\
175.0241\end{array}$ & Hesperetin-glucuronide & 7.46 & 7.52 & 25.25 & $2 . \approx$ \\
\hline M50 & 21.17 & {$[\mathrm{M}-\mathrm{H}]^{-}$} & 621.1130 & 621.1097 & 5.3 & $\mathrm{C}_{27} \mathrm{H}_{26} \mathrm{O}_{17}$ & $\begin{array}{l}\text { 445.0783; } \\
383.0786 ; \\
269.0459 \\
175.0243 \\
113.0232\end{array}$ & Baicalein-di-glucuronide & 70.49 & 11.47 & 26.47 & 1.C \\
\hline M51 & 21.17 & {$[\mathrm{M}-\mathrm{H}]^{-}$} & 379.0120 & 379.0129 & -2.4 & $\mathrm{C}_{16} \mathrm{H}_{12} \mathrm{O}_{9} \mathrm{~S}$ & $\begin{array}{l}380.1751 \\
299.0564 \\
284.0330 \\
229.0178 \\
201.1126 \\
124.0060\end{array}$ & $\begin{array}{l}\text { Wogonin-sulfate- } \\
\text { hydroxylation }\end{array}$ & 26.81 & 32.52 & 143.23 & 15 \\
\hline M52 & 21.17 & {$[\mathrm{M}-\mathrm{H}]^{-}$} & 635.1234 & 635.1253 & -3.0 & $\mathrm{C}_{28} \mathrm{H}_{28} \mathrm{O}_{17}$ & $\begin{array}{l}\text { 459.0945; } \\
325.0716 ; \\
283.0615 \\
268.0378 \\
175.0239\end{array}$ & $\begin{array}{l}\text { Wogonin-di-glucuronide } \\
\text { or Oroxylin A-di- } \\
\text { glucuronide }\end{array}$ & 3.56 & 0.29 & 3.36 & $0 . c$ \\
\hline M53 & 21.20 & {$[\mathrm{M}-\mathrm{H}]^{-}$} & 167.0341 & 167.0350 & 1.286 & $\mathrm{C}_{8} \mathrm{H}_{8} \mathrm{O}_{4}$ & 167.0341 & Vanillic acid isomer & 4.30 & 0.00 & 2.86 & $0 . \mathrm{C}$ \\
\hline M54 & 21.35 & {$[\mathrm{M}-\mathrm{H}]^{-}$} & 461.0702 & 461.0725 & -5.1 & $\mathrm{C}_{21} \mathrm{H}_{18} \mathrm{O}_{12}$ & $\begin{array}{l}285.0400 \\
229.0507 \\
\text { 195.0445; } \\
85.0288\end{array}$ & $\begin{array}{l}\text { Trihydroxyflavone- } \\
\text { glucuronide }\end{array}$ & 2.12 & 0.88 & 2.19 & $0 . c$ \\
\hline M55 & 21.42 & $\begin{array}{l}{[\mathrm{M}+} \\
\mathrm{H}]^{+}\end{array}$ & 609.1439 & 609.1509 & -11.5 & $\mathrm{C}_{27} \mathrm{H}_{28} \mathrm{O}_{16}$ & $\begin{array}{l}573.6403 \\
447.0903 \\
314.0520 \\
271.0596 \\
226.4313\end{array}$ & Baicalein-di-glucuronide & 1.42 & 1.46 & 9.22 & $0 . c$ \\
\hline M56 & 21.46 & $\begin{array}{l}{[\mathrm{M}+} \\
\mathrm{H}]^{+}\end{array}$ & 623.1241 & 623.1243 & -0.3 & $\mathrm{C}_{27} \mathrm{H}_{26} \mathrm{O}_{17}$ & $\begin{array}{l}\text { 447.0887; } \\
285.0754 \\
271.0595\end{array}$ & $\begin{array}{l}\text { Norwogonin-di- } \\
\text { glucuronide }\end{array}$ & 9.74 & 1.76 & 2.22 & $0 . c$ \\
\hline M57\# & 21.72 & {$[\mathrm{M}-\mathrm{H}]^{-}$} & 381.0270 & 381.0286 & -4.2 & $\mathrm{C}_{16} \mathrm{H}_{14} \mathrm{O}_{9} \mathrm{~S}$ & $\begin{array}{l}381.0279 ; \\
301.0725 ; \\
286.0487 ; \\
229.0175 ; \\
193.0347\end{array}$ & Hesperetin-sulfate & 0.80 & 3.40 & 8.25 & $0 . c$ \\
\hline M58 & 21.73 & {$[\mathrm{M}-\mathrm{H}]^{-}$} & 525.0308 & 525.0344 & -7.0 & $\mathrm{C}_{21} \mathrm{H}_{18} \mathrm{O}_{14} \mathrm{~S}$ & $\begin{array}{l}525.2744 ; \\
445.0775 ; \\
349.0027 ; \\
269.0459 ; \\
175.0241\end{array}$ & Baicalin-sulfate & 12.25 & 0.00 & 52.63 & $0 . C$ \\
\hline M59\# & 22.04 & {$[\mathrm{M}-\mathrm{H}]^{-}$} & 477.1038 & 477.1038 & 0.0 & $\mathrm{C}_{22} \mathrm{H}_{22} \mathrm{O}_{12}$ & $\begin{array}{l}477.1039 \\
415.1037 \\
301.0722 \\
257.0823 \\
215.0710 \\
162.0221\end{array}$ & Hesperetin-glucuronide & 4.03 & 9.80 & 24.65 & 2.4 \\
\hline
\end{tabular}




\begin{tabular}{|c|c|c|c|c|c|c|c|c|c|c|c|c|}
\hline \multirow[t]{2}{*}{ NO } & \multirow{2}{*}{$\begin{array}{l}\mathrm{RT} \\
\text { /min }\end{array}$} & \multirow{2}{*}{$\begin{array}{l}\text { lon } \\
\text { model }\end{array}$} & \multirow{2}{*}{$\begin{array}{l}\text { Measured } \\
\text { mass /Da }\end{array}$} & \multirow{2}{*}{$\begin{array}{l}\text { Calculated } \\
\text { mass / } \mathrm{Da}\end{array}$} & \multirow{2}{*}{$\begin{array}{l}\text { Error } \\
\text { /ppm }\end{array}$} & \multirow{2}{*}{$\begin{array}{l}\text { Molecular } \\
\text { formula }\end{array}$} & \multirow{2}{*}{$\begin{array}{l}\text { Fragment } \\
\text { ions }\end{array}$} & \multirow[t]{2}{*}{ Identification } & \multicolumn{4}{|c|}{ Peak Area $\left({ }^{\star} 10^{6}\right)$} \\
\hline & & & & & & & & & Serum & Lung & Liver & $\mathrm{He}$ \\
\hline M60 & 22.04 & {$[\mathrm{M}-\mathrm{H}]^{-}$} & 503.1166 & 503.1195 & -5.8 & $\mathrm{C}_{24} \mathrm{H}_{24} \mathrm{O}_{12}$ & $\begin{array}{l}354.9995 \\
269.0461\end{array}$ & $\begin{array}{l}\text { Isoscutellarein- } \\
\text { glucuronide }\end{array}$ & 1.03 & 0.62 & 2.30 & $0 . c$ \\
\hline M61 & 22.14 & {$[\mathrm{M}-\mathrm{H}]^{-}$} & 635.1256 & 635.1253 & 0.5 & $\mathrm{C}_{28} \mathrm{H}_{28} \mathrm{O}_{17}$ & $\begin{array}{l}\text { 459.0936; } \\
325.0712 ; \\
283.0615 ; \\
268.0380 ; \\
175.0240\end{array}$ & $\begin{array}{l}\text { Isowogonin-di- } \\
\text { glucuronide }\end{array}$ & 10.28 & 1.19 & 3.36 & $0 . c$ \\
\hline M62 & 22.36 & {$[\mathrm{M}-\mathrm{H}]^{-}$} & 461.0698 & 461.0725 & -6.0 & $\mathrm{C}_{21} \mathrm{H}_{18} \mathrm{O}_{12}$ & $\begin{array}{l}\text { 285.0441; } \\
\text { 139.0062; } \\
61.9885\end{array}$ & $\begin{array}{l}\text { Trihydroxyflavone- } \\
\text { glucuronide }\end{array}$ & 0.65 & 0.12 & 0.00 & $0 . C$ \\
\hline M63 & 22.45 & $\begin{array}{l}{[\mathrm{M}+} \\
\mathrm{H}]^{+}\end{array}$ & 609.1440 & 609.1509 & -11.3 & $\mathrm{C}_{20} \mathrm{H}_{32} \mathrm{O}_{21}$ & $\begin{array}{l}573.6403 \\
447.0903 ; \\
314.0520 ; \\
271.0596 \\
226.4313\end{array}$ & $\begin{array}{l}\text { Baicalein-glucosyl- } \\
\text { glucuronide }\end{array}$ & 0.27 & 7.93 & 4.34 & $0 . c$ \\
\hline M64 & 22.63 & {$[\mathrm{M}-\mathrm{H}]^{-}$} & 651.1189 & 651.1203 & -2.2 & $\mathrm{C}_{28} \mathrm{H}_{28} \mathrm{O}_{18}$ & $\begin{array}{l}\text { 475.0886; } \\
475.0886 ; \\
299.0565 ; \\
284.0330 ; \\
175.0241\end{array}$ & $\begin{array}{l}\text { Trihydroxy- } \\
\text { methoxyflavone-di- } \\
\text { glucuronide }\end{array}$ & 3.37 & 0.20 & 0.18 & $0 . C$ \\
\hline M65\# & 22.64 & {$[\mathrm{M}-\mathrm{H}]^{-}$} & 431.0969 & 431.0983 & -3.2 & $\mathrm{C}_{21} \mathrm{H}_{20} \mathrm{O}_{10}$ & $\begin{array}{l}255.0703 \\
135.0099 \\
119.0503\end{array}$ & $\begin{array}{l}\text { Liquiritigenin- } \\
\text { glucuronide }\end{array}$ & 4.76 & 0.00 & 17.17 & $0 . c$ \\
\hline M66 & 22.85 & {$[\mathrm{M}-\mathrm{H}]^{-}$} & 505.0960 & 505.0987 & -5.3 & $\mathrm{C}_{23} \mathrm{H}_{22} \mathrm{O}_{13}$ & $\begin{array}{l}505.0964 ; \\
428.0726 ; \\
329.0672 ; \\
314.0437 ; \\
299.0199\end{array}$ & $\begin{array}{l}\text { Trihydroxy- } \\
\text { dimethoxyflavone- } \\
\text { glucuronide }\end{array}$ & 47.70 & 24.23 & 72.26 & 9.5 \\
\hline M67 & 22.87 & {$[\mathrm{M}-\mathrm{H}]^{-}$} & 349.0008 & 349.0023 & -4.5 & $\mathrm{C}_{15} \mathrm{H}_{10} \mathrm{O}_{8} \mathrm{~S}$ & $\begin{array}{l}307.1511 ; \\
269.0459 \\
252.0426 \\
207.1397\end{array}$ & Baicalein-sulfate & 25.59 & 30.98 & 305.56 & 10 \\
\hline M68 & 23.62 & {$[\mathrm{M}-\mathrm{H}]^{-}$} & 533.1306 & 533.1300 & 1.1 & $\mathrm{C}_{25} \mathrm{H}_{26} \mathrm{O}_{13}$ & $\begin{array}{l}357.0987 \\
327.0515 \\
309.0418 \\
175.0238\end{array}$ & $\begin{array}{l}\text { Sinensetin-glucuronide } \\
\text { demethylation }\end{array}$ & 3.42 & 4.15 & 20.20 & 1.7 \\
\hline M69\# & 23.74 & {$[\mathrm{M}-\mathrm{H}]^{-}$} & 347.0221 & 347.0231 & -2.9 & $\mathrm{C}_{16} \mathrm{H}_{12} \mathrm{O}_{7} \mathrm{~S}$ & $\begin{array}{l}347.1715 ; \\
269.0457 \\
232.0168 \\
\text { 193.0348; } \\
\text { 173.0997; } \\
113.0232\end{array}$ & Formononetin-sulfate & 17.66 & 1.36 & 7.99 & $0 . c$ \\
\hline M70\# & 23.74 & {$[\mathrm{M}-\mathrm{H}]^{-}$} & 335.0216 & 335.0231 & -4.5 & $\mathrm{C}_{15} \mathrm{H}_{12} \mathrm{O}_{7} \mathrm{~S}$ & $\begin{array}{l}255.0897 \\
135.0104 \\
119.0511\end{array}$ & Liquiritigenin-sulfate & 3.63 & 2.50 & 2.04 & 4.2 \\
\hline M71 & 24.06 & {$[\mathrm{M}-\mathrm{H}]^{-}$} & 563.1377 & 563.1406 & -5.1 & $\mathrm{C}_{26} \mathrm{H}_{28} \mathrm{O}_{14}$ & $\begin{array}{l}387.1091 ; \\
372.0855 ; \\
357.0622 ; \\
342.0382 ; \\
215.0739\end{array}$ & $\begin{array}{l}\text { Nobiletin-glucuronide- } \\
\text { demethylation }\end{array}$ & 2.55 & 5.71 & 27.24 & 2.4 \\
\hline M72\# & 24.16 & {$[\mathrm{M}-\mathrm{H}]^{-}$} & 349.0003 & 349.0023 & -5.9 & $\mathrm{C}_{15} \mathrm{H}_{10} \mathrm{O}_{8} \mathrm{~S}$ & $\begin{array}{l}269.0516 \\
229.0295 \\
117.0386\end{array}$ & Baicalein-sulfate & 20.92 & 25.15 & 95.15 & $2 . \epsilon$ \\
\hline M73 & 24.38 & $\begin{array}{l}{[\mathrm{M}+} \\
\mathrm{H}]^{+}\end{array}$ & 285.0739 & 285.0758 & -6.5 & $\mathrm{C}_{16} \mathrm{H}_{12} \mathrm{O}_{5}$ & $\begin{array}{l}285.0753 ; \\
270.0518 ; \\
249.1475 ; \\
221.1528 ; \\
183.0287\end{array}$ & $\begin{array}{l}\text { Baicalein- } \\
\text { methoxylation }\end{array}$ & 5.36 & 0.29 & 23.62 & 2.5 \\
\hline M74 & 24.6 & {$[\mathrm{M}-\mathrm{H}]^{-}$} & 475.0858 & 475.0882 & -5.1 & $\mathrm{C}_{22} \mathrm{H}_{20} \mathrm{O}_{12}$ & $\begin{array}{l}\text { 299.0565; } \\
284.0330 ; \\
204.0077 ; \\
113.0231 ; \\
85.0281\end{array}$ & $\begin{array}{l}\text { Trihydroxy- } \\
\text { methoxyflavone- } \\
\text { glucuronide }\end{array}$ & 6.49 & 20.09 & 21.93 & 1.2 \\
\hline M75 & 24.64 & $\begin{array}{l}{[\mathrm{M}+} \\
\mathrm{H}]^{+}\end{array}$ & 521.1271 & 521.1290 & -3.6 & $\mathrm{C}_{24} \mathrm{H}_{24} \mathrm{O}_{13}$ & $\begin{array}{l}521.1315 \\
345.0964 ; \\
330.0723 \\
284.0662 \\
205.0488 \\
85.0286\end{array}$ & $\begin{array}{l}\text { Skullcapflavon I- } \\
\text { glucuronide }\end{array}$ & 1.27 & 0.29 & 3.13 & $0 . c$ \\
\hline
\end{tabular}




\begin{tabular}{|c|c|c|c|c|c|c|c|c|c|c|c|c|}
\hline \multirow[t]{2}{*}{ No } & \multirow{2}{*}{$\begin{array}{l}\mathrm{RT} \\
/ \mathrm{min}\end{array}$} & \multirow{2}{*}{$\begin{array}{l}\text { lon } \\
\text { model }\end{array}$} & \multirow{2}{*}{$\begin{array}{l}\text { Measured } \\
\text { mass / } \mathrm{Da}\end{array}$} & \multirow{2}{*}{$\begin{array}{l}\text { Calculated } \\
\text { mass /Da }\end{array}$} & \multirow{2}{*}{$\begin{array}{l}\text { Error } \\
\text { /ppm }\end{array}$} & \multirow{2}{*}{$\begin{array}{l}\text { Molecular } \\
\text { formula }\end{array}$} & \multirow{2}{*}{$\begin{array}{l}\text { Fragment } \\
\text { ions }\end{array}$} & \multirow[t]{2}{*}{ Identification } & \multicolumn{4}{|c|}{ Peak Area $\left({ }^{*} 10^{6}\right)$} \\
\hline & & & & & & & & & Serum & Lung & Liver & $\mathrm{He}$ \\
\hline M76 & 24.69 & $\begin{array}{l}{[\mathrm{M}+} \\
\mathrm{H}]^{+}\end{array}$ & 491.1160 & 491.1184 & -4.9 & $\mathrm{C}_{23} \mathrm{H}_{22} \mathrm{O}_{12}$ & $\begin{array}{l}\text { 491.0766; } \\
316.0604 \\
285.0440\end{array}$ & $\begin{array}{l}\text { Wogonoside- } \\
\text { methoxylation }\end{array}$ & 0.59 & 0.00 & 0.00 & $0 . c$ \\
\hline M77 & 24.75 & {$[\mathrm{M}-\mathrm{H}]^{-}$} & 299.0560 & 299.0561 & -0.4 & $\mathrm{C}_{16} \mathrm{H}_{12} \mathrm{O}_{6}$ & $\begin{array}{l}299.0567 ; \\
284.0331 \\
236.9977 \\
219.0774 \\
175.0756\end{array}$ & Wogonin-hydroxylation & 8.84 & 49.84 & 261.36 & 15 \\
\hline M78 & 25.07 & $\begin{array}{l}{[\mathrm{M}+} \\
\mathrm{H}]^{+}\end{array}$ & 461.1075 & 461.1078 & -0.7 & $\mathrm{C}_{22} \mathrm{H}_{20} \mathrm{O}_{11}$ & $\begin{array}{l}285.0753 \\
270.0517 \\
153.0180 \\
113.0235\end{array}$ & $\begin{array}{l}\text { Baicalein-glucuronide } \\
\text { methylation }\end{array}$ & 98.36 & 0.00 & 1328.19 & 21 \\
\hline M79 & 25.07 & $\begin{array}{l}{[\mathrm{M}+} \\
\mathrm{H}]^{+}\end{array}$ & 285.0741 & 285.0758 & -5.8 & $\mathrm{C}_{16} \mathrm{H}_{12} \mathrm{O}_{5}$ & $\begin{array}{l}285.0753 \\
270.0518 ; \\
183.0287 \\
167.0936 \\
133.1007\end{array}$ & $\begin{array}{l}\text { Baicalein- } \\
\text { methoxylation }\end{array}$ & 11.72 & 87.37 & 137.38 & 10 \\
\hline M80\# & 25.73 & {$[\mathrm{M}-\mathrm{H}]^{-}$} & 363.0180 & 363.0180 & 0.0 & $\mathrm{C}_{16} \mathrm{H}_{12} \mathrm{O}_{8} \mathrm{~S}$ & $\begin{array}{l}283.0616 \\
268.0381 \\
163.0034 \\
137.0236\end{array}$ & Wogonin-sulfate & 35.90 & 54.16 & 98.83 & 25 \\
\hline M81\# & 26.31 & {$[\mathrm{M}-\mathrm{H}]^{-}$} & 387.1066 & 387.1085 & -4.9 & $\mathrm{C}_{20} \mathrm{H}_{20} \mathrm{O}_{8}$ & $\begin{array}{l}357.0632 ; \\
342.0382 ; \\
268.0370 ; \\
162.9988 \\
78.9593\end{array}$ & Nobiletin-demethylation & 0.56 & 4.11 & 38.96 & 2.2 \\
\hline M82\# & 26.36 & {$[\mathrm{M}-\mathrm{H}]^{-}$} & 363.0176 & 363.0180 & -1.1 & $\mathrm{C}_{16} \mathrm{H}_{12} \mathrm{O}_{8} \mathrm{~S}$ & $\begin{array}{l}283.0610 \\
268.0375 \\
239.0374\end{array}$ & Wogonin-sulfate & 35.94 & 35.58 & 193.63 & 13 \\
\hline M83 & 26.76 & {$[\mathrm{M}-\mathrm{H}]^{-}$} & 529.1335 & 529.1351 & -3.1 & $\mathrm{C}_{26} \mathrm{H}_{26} \mathrm{O}_{12}$ & $\begin{array}{l}353.1036 \\
329.1607 \\
273.0777 \\
125.0232\end{array}$ & $\begin{array}{l}\text { Licoisoflavone A- } \\
\text { glucuronide }\end{array}$ & 5.92 & 1.46 & 8.46 & $0 . c$ \\
\hline M84 & 27.39 & {$[\mathrm{M}-\mathrm{H}]^{-}$} & 529.1343 & 529.1351 & -1.6 & $\mathrm{C}_{26} \mathrm{H}_{26} \mathrm{O}_{12}$ & $\begin{array}{l}353.1011 \\
\text { 125.0248; } \\
61.9896\end{array}$ & $\begin{array}{l}\text { Licoisoflavone A- } \\
\text { glucuronide }\end{array}$ & 0.20 & 0.19 & 0.00 & $0 . c$ \\
\hline M85 & 27.76 & {$[\mathrm{M}-\mathrm{H}]^{-}$} & 527.1169 & 527.1195 & -4.9 & $\mathrm{C}_{26} \mathrm{H}_{24} \mathrm{O}_{12}$ & $\begin{array}{l}527.2500 \\
351.0862 \\
323.0813 \\
151.0048\end{array}$ & $\begin{array}{l}\text { Semilicoisoflavone B- } \\
\text { glucuronide }\end{array}$ & 0.15 & 0.88 & 1.60 & $0 . c$ \\
\hline M86\# & 27.89 & {$[\mathrm{M}-\mathrm{H}]^{-}$} & 387.1066 & 387.1085 & -4.9 & $\mathrm{C}_{20} \mathrm{H}_{20} \mathrm{O}_{8}$ & $\begin{array}{l}357.0632 \\
342.0382 \\
268.0370 \\
162.9988 \\
78.9593\end{array}$ & Nobiletin-demethylation & 2.11 & 0.00 & 1.58 & 1.1 \\
\hline M87 & 28.17 & {$[\mathrm{M}-\mathrm{H}]^{-}$} & 527.1160 & 527.1195 & -6.6 & $\mathrm{C}_{26} \mathrm{H}_{24} \mathrm{O}_{12}$ & $\begin{array}{l}527.2577 \\
351.0877 \\
283.1005\end{array}$ & $\begin{array}{l}\text { Semilicoisoflavone B- } \\
\text { glucuronide }\end{array}$ & 0.39 & 0.00 & 0.71 & $0 . c$ \\
\hline M88 & 29.1 & {$[\mathrm{M}-\mathrm{H}]^{-}$} & 253.0511 & 253.0506 & 2.0 & $\mathrm{C}_{15} \mathrm{H}_{10} \mathrm{O}_{4}$ & $\begin{array}{l}\text { 253.0508; } \\
\text { 207.1388; } \\
\text { 189.1281; } \\
\text { 163.0760; } \\
143.0488\end{array}$ & $\begin{array}{l}\text { Liquiritigenin- } \\
\text { dehydrogenation }\end{array}$ & 0.42 & 33.04 & 297.05 & 10 \\
\hline M89\# & 29.32 & $\begin{array}{l}{[\mathrm{M}+} \\
\mathrm{H}]^{+}\end{array}$ & 285.0749 & 285.0758 & -3.0 & $\mathrm{C}_{16} \mathrm{H}_{12} \mathrm{O}_{5}$ & $\begin{array}{l}\text { 285.0753; } \\
270.0517 \\
201.1260 ; \\
183.0276 ; \\
159.1165\end{array}$ & $\begin{array}{l}\text { Baicalein- } \\
\text { methoxylation }\end{array}$ & 7.78 & 90.10 & 526.55 & 84 \\
\hline M90 & 29.36 & $\begin{array}{l}{[\mathrm{M}+} \\
\mathrm{H}]^{+}\end{array}$ & 527.3324 & 527.3367 & -8.2 & $\mathrm{C}_{32} \mathrm{H}_{46} \mathrm{O}_{6}$ & $\begin{array}{l}\text { 527.3339; } \\
\text { 509.3266; } \\
\text { 407.1786; } \\
\text { 375.1550; } \\
290.3741\end{array}$ & $\begin{array}{l}\text { Alisol C-23 acetate- } \\
\text { dehydrogenation }\end{array}$ & 0.20 & 8.71 & 48.49 & $0 . c$ \\
\hline M91 & 34.45 & {$[\mathrm{M}-\mathrm{H}]^{-}$} & 485.3241 & 485.3272 & -6.5 & $\mathrm{C}_{30} \mathrm{H}_{46} \mathrm{O}_{5}$ & $\begin{array}{l}485.3303 \\
467.2944 \\
439.3218 \\
423.3270\end{array}$ & $\begin{array}{l}22 \text {-acetoxyl } \\
\text { glycyrrhizin- } \\
\text { deacetylated- } \\
\text { dediglucuronide }\end{array}$ & 2.79 & 0.52 & 9.36 & $0 . c$ \\
\hline
\end{tabular}


M- means metabolite, \#- means the metabolite that has been confirmed by microsomal incubation in vitro; RT-retention time.

\subsection{Characterization of the absorbed prototypes and metabolites in mice tissues}

With the help of the information about the absorbed prototypes and metabolites in mice serum, the absorbed prototypes and metabolites in various tissues from QFPDD-treated mice were explored. Subsequently, total of $165,177,112,120,44$ and 53 prototypes and metabolites were identified in the lung, liver, heart, kidney, brain, and spleen of QFPDD-treated mice, respectively. The peak areas of absorbed prototypes and metabolites were summarized in Table 2 and Table 3, while Fig. 6 showed the heat map of the peak areas of both the absorbed components and the metabolites occurring in serum or six different tissues of QFPDD-treated mice.

On the basis of the peak area, the top ten components in the mice serum were ranked as follows, stachydrine (p3), salicylic acid (p32), ephedrine (p7), caffeic acid-sulfate (M4), baicalin (p66), baicalein 6-O- $\beta$-D-glucuronide (p82), wogonoside (p80), methylephedrine (p10), baicalein-glucuronide methylation (M78), quinic acid (p2).

The top ten components in the lung tissue were ranked as follows: ephedrine (p7), naringin (p53), neohesperidin (p59), baicalin (p66), wogonoside (p80), stachydrine (p3), quinic acid (p2), methylephedrine (p10), oroxylin A 7-O-glucuronide (p75), pseudoephedrine (p9).

The top ten components in the liver tissue were ranked as follows: ephedrine (p7), naringin (p53), neohesperidin (p59), baicalein-glucuronide methylation (M78), irisflorentin (p87), stachydrine (p3), wogonoside (p80), wogonin (p88), baicalin (p66), methylephedrine (p10).

The top ten components in the heart tissue were ranked as follows: ephedrine (p7), naringin (p53), stachydrine (p3), methylephedrine (p10), neohesperidin (p59), alisol 0 (p102), wogonoside (p80), pseudoephedrine (p9), baicalein-methoxylation (M79), alisol A (p103).

The top ten components in the kidney tissue were ranked as follows: ephedrine (p7), naringin (p53), neohesperidin (p59), baicalin (p66), wogonoside (p80), stachydrine (p3), methylephedrine (p10), quinic acid (p2), oroxylin A 7-0-glucuronide (p75), pseudoephedrine (p9).

The top ten components in the brain tissue were ranked as follows: ephedrine (p7), pseudoephedrine (p9), methylephedrine (p10), stachydrine (p3), mandelonitrile (M1), phenylpropanolamine (p4), salicylic acid (p32), alisol O (p102), D-demethyl pseudoephedrine (p5), oroxylin A (p93).

The top ten components in the spleen tissue were ranked as follows: ephedrine (p7), stachydrine (p3), methylephedrine (p10), pseudoephedrine (p9), oroxylin A (p93), baicalein -methoxylation (M89), pinellic acid isomer (p96), alisol 0 (p102), wogonin (p88).

From the above results, it was obvious that ephedrine (p7) and methylephedrine (p10) from Herba Ephedrae, and stachydrine (p3) from Fructus Aurantii Immaturus displayed relatively high exposure levels in mice serum and all tested tissues. Additionally, baicalin (p66), oroxylin A 7-0-glucuronide (p75), wogonoside (p80), wogonin (p88) and oroxylin A (p93) from Radix Scutellariae, alisol 0 (p102) and alisol A (p103) from Rhizoma Alismatis also showed relatively high exposure levels in most of mice tissues.

\section{Discussion}

To fight against COVID-19, some Chinese medicines were recommended by National Health Commission of the People's Republic of China or the local government in China, for alleviating the major symptoms of COVID-19 or preventing disease deterioration [9]. As the first Chinese medicine compound formula recommended for combating COVID-19, QFPDD is made by 20 herbs and a mineral drug, which is composed by hundreds of ingredients. In most cases, for treating COVID-19, the absorbed constituents (including the prototypes and the metabolites) into blood circulation can be delivered to the target organ (such as the lung) and then exerts various pharmacological effects, such as anti-inflammatory and anticoagulant effects [23, 24]. Therefore, it is necessary to investigate the absorbed constituents of QFPDD following oral administration of this super Chinese medicine compound formula, which will be very helpful for better understanding the key material basis of QFPDD, as well as for guiding the quality control and clinical applications of this formula.

In this study, an UHPLC-Q-Orbitrap HRMS based method was established for profiling the constituents in QFPDD and the absorbed constituents in QFPDDtreated mice. The research strategy and the key findings for clarifying the chemical composition and absorption components of QFPDD have been summarized in Fig. 7. The chemical constituents in QFPDD were tentatively identified via comparison with exact molecular weight, the retention times and MS/MS spectra of the standards or refereed by TCM databases and literature. The absorbed components (including the prototypes and the metabolites) occurring in mice serum and tissues were identified via profiling the serum and tissue samples from QFPDD-treated mice. The absorbed prototype components were tentatively identified via comparison with the retention times and MS/MS spectra of components in QFPDD. The in vivo metabolites were tentatively identified via comparison with fragment ions of the parent compounds or refereed by literature, while parts of in vivo metabolites of the major constituents were identified via comparison with the retention times and MS/MS spectra of the in vitro metabolites of each constituent generated in MLMs. Finally, a total of 405 chemicals (including alkaloids, flavonoids, organic acids, triterpene saponins and other compounds) in the extract of QFPDD were identified, while 195, 165, 177, 112, 120, 44, 53 constituents were identified in the serum, lung, liver, heart, kidney, brain, and spleen of QFPDD-treated mice, respectively. All these findings might be very helpful for deep understanding the fates of the constituents in QFPDD, as well as offered key information of the tissue distribution of the absorbed components of this anti-COVID-19 Chinese medicine.

It is well known that it is always a challenging task for identification of the prototypes and the metabolites of TCM prescription. For example, oroxylin A 7-Oglucuronide (p75) is a trace amount prototype constituent occurring in QFPDD, but this agent is a highly exposed component in the serum and various tissues of QFPDD-treated mice. The reasons may include i) produced by the O-glucuronidation of oroxylin A (p103) in vivo; ii) produced by baicalein (p66) through 6O-methylation and $O$-glucuronidation. It is also suggested that it is very difficult to differentiate some components detected in QFPDD-treated mice as the prototypes or the metabolites, owing to that some in vivo components are derived from both the prototypes and the metabolites of the homologous 
compounds occurring in this TCM prescription. Meanwhile, the exposure of some prototype constituents in blood are extremely low and thus are difficult to be detected, since these natural constituents could be readily transformed into the corresponding metabolites (glucuronides or sulfates) in vivo. For instances, trihydroxy-methoxyflavone-O-glucuronides (M35 and M60) and daidzein-sulfates (M33 and M41) can be easily detected in mice serum, but the related prototypes are hardly detected in mice serum. In addition, many nature products have isomers that cannot be distinguished by MS spectral analysis alone, thus it is hard to correlate the parent compounds with the metabolites, such as vanillic acid and its isomer.

Modern pharmacological studies have found that some of absorbed components of QFPDD displayed a wide range of benificical effects or biological activities. Some pharmacological activities of them are considered to be beneficial for COVID-19 patients. For example, ephedrine, pseudoephedrine and amygdalin displayed significant anti-cough and anti-asthmatic effects [25], as well as anti-inflammatory [26, 27] and immunomodulatory effects [28, 29], which are considered as the major active components of TCM prescriptions for treating respiratory diseases. Zhang et al. [30] report that amygdalin can induce LPS by inhibiting NF-KB and NLRP3 signaling pathways, while baicalin has antiviral, anti-inflammatory, antioxidant, and immunomodulatory pharmacological activities [31]. A recent study has reported that baicalin can significantly inhibit the catalytic activity of SARS-CoV-2 3CL pro and then exert antiviral activity [32]. The virtual screening of active ingredients of SARS-CoV-2 virus also suggests that hesperidin and baicalin have potential inhibitory effects [33]. The glycyrrhizin and glycyrrhizic acid in licorice have been found with extensive anti-inflammatory [34] and anti-damage effects [35]. Some previous studies have shown that glycyrrhizin can serve as an alternative agent for treating COVID-19 infection and its associated respiratory syndrome [3638]. The extract of Rhizoma Alismatis processed exerts anti-inflammatory activity via inhibiting cytochrome P450 enzymes [39], the key enzymes responsible for the metabolism of arachidonic acid, thereby reducing the production of inflammatory factors [40-42].

Furthermore, some in vivo metabolites of QFPDD are more likely to be the active ingredients. Previous studies clearly demonstrated that a panel of $O$ glucuronides of flavonoids displayed good anticoagulant activities, and their anticoagulant activities are better than that of the prototypes [43]. Recent studies have shown that the blood of COVID-19 patients was in a hypercoagulable state, thus the O-glucuronides of flavonoids were more likely to promote blood circulation through anticoagulation and then relieve the symptoms of patients [44-46]. This study was not only found a considerable number of prototypes and metabolites in serum, but also found a large number of chemical components in lung, liver and other tissues. Among them, the active ingredients derived from Herba Ephedrae (ephedrine, pseudoephedrine, methylephedrine, phenylpropanolamine etc.), Fructus Aurantii Immaturus (stachydrine, naringin, hesperidin, neohesperidin, quinic acid etc.), Radix Scutellariae (baicalin, oroxylin A 7-O-glucuronide, wogonoside, wogonin, oroxylin A etc.) and Rhizoma Alismatis (alisol $\mathrm{O}$, alisol A etc.) displayed relatively high exposure levels in most of organs. However, the kinds and exposure levels of each component in various tissues are much different. These findings suggest that these in vivo metabolites of QFPDD may play potential role in the treatment of COVID-19 via targeting multiple targets or pathways. In future, the bioactive ingredients and mechanisms of action can be explored.

\section{Conclusion}

In summary, a practical and sensitive UHPLC-Q-Exactive-Orbitrap HRMS approach was developed for chemical profiling of the constituents in QFPDD, as well as the absorbed prototypes and the metabolites in mice serum and tissues following oral administration of QFPDD. With the help of the authentic standards and in vitro metabolites generated by MLM, a total of 405 constituents (including 40 kinds of alkaloids, 162 kinds of flavonoids, 44 kinds of organic acids, 71 kinds of triterpene saponins and 88 kinds of other compounds) were identified from the water extract of QFPDD, while 195 components (including 104 prototypes and 91 metabolites) were identified from mice serum after oral administration of QFPDD to mice. Meanwhile, 165, 177, 112, 120, 44, 53 components were identified from the lung, liver, heart, kidney, brain, and spleen of QFPDD-treated mice, respectively. Additionally, the metabolic pathways of some major absorbed components occurring in the serum of QFPDD-treated mice were also described. All these findings provided key information and guidance for further investigations on the pharmacologically active substances and quality control of QFPDD.

\section{Abbreviations}

CMs, Chinese Medicines; COVID-19, Corona Virus Disease 2019; CYPs/P450s, cytochrome P450 enzymes; ESI, electrospray ionization; G-6-P, D-Glucose-6phosphate; G-6-PDH, glucose-6-phosphate dehydrogenase;

MLMs, mice liver microsomes; $\beta$-NADP ${ }^{+}$, nicotinamide adenine dinucleotide phosphate disodium salt; PAPS, 3-phosphoadenosine 5-phosphosulfate; PBS, potassium phosphate buffer; QFPDD, Qingfei Paidu decoction; SAM, Sadenosyl-L-methionine; SARS, Severe Acute Respiratory Syndrome; UDPGA, uridine diphosphate glucuronic acid; UHPLC-Q-Orbitrap HRMS, ultra-high-performance liquid chromatography-Q exactive hybrid quadrupole orbitrap high-resolution accurate mass spectrometric.

\section{Declarations}

\section{Acknowledgements}

We thank Asso. Prof. Caisheng Wu from Xiamen University, for his help in identification of prototype constituents and metabolites in mice serum following oral administration of QFPDD to mice.

\section{Authors' contributions}

WL and JH carried out the experiments; WL, FZ, and GG wrote the manuscript and analyzed the data; WL, FZ, CZ and RL analyzed the data; $C W$ prepared the extract of QFPDD; GG revised the manuscript; GG, WZ and PL conceived of the study, contributed to the design and interpretation of the research. All authors read and approved the fnal manuscript. 


\section{Funding}

This work was supported by the National Key Research and Development Program of China (2020YFC0845400, 2017YFC1700200, 2017YFC1702000), the NSF of China (81922070, 81973286, 8170368, 81530101), Shanghai Science and Technology Innovation Action Plans (20S21901500ه20S21900900) supported by Shanghai Science and Technology Committee, the Three-year Action Plan of Shanghai TCM Development (ZY-(2018-2020)-CCCX-5001), Program of Shanghai Academic/Technology Research Leader (18XD1403600), Shuguang Program (18SG40) \& the Project on the Prevention and Treatment of COVID-19 with Chinese and Western Medicines that supported by Shanghai Education Development Foundation and Shanghai Municipal Education Commission.

\section{Availability of data and materials}

The research data generated from this study is included within the article.

\section{Ethics approval and consent to participate}

Animal experimental was ratified by the Animal Care and Use Committee of Shanghai Institute of Food and Drug Control.

\section{Consent for publication}

Not applicable.

\section{Competing interests}

The authors declare that they have no competing interests.

\section{Author details}

${ }^{1}$ Key Laboratory of Liver and Kidney Diseases (Ministry of Education), Institute of Liver Diseases, Shanghai Key Laboratory of Traditional Chinese Clinical Medicine, Shuguang Hospital Affiliated to Shanghai University of Traditional Chinese Medicine, Shanghai 201203, China. ${ }^{2}$ Institute of Interdisciplinary Integrative Medicine Research, Shanghai University of Traditional Chinese Medicine, Shanghai 201203, China. ${ }^{3}$ Institute of Chinese Materia Medica, Shanghai University of Traditional Chinese Medicine, Shanghai 201203, China. ${ }^{4}$ Dalian Institute of Chemical Physics, Chinese Academy of Sciences, Dalian, 116023 , China.

\section{References}

1. Hamidian Jahromi A, Hamidianjahromi A. Why African Americans Are a Potential Target for COVID-19 Infection in the United States. J Med Internet Res. 2020;22:e19934.

2. Dima M, Enatescu I, Craina M, Petre I, lacob ER, lacob D. First neonates with severe acute respiratory syndrome coronavirus 2 infection in Romania: Three case reports. Medicine (Baltimore). 2020;99:e21284.

3. Niu S, Tian S, Lou J, Kang X, Zhang L, Lian H, Zhang J. Clinical characteristics of older patients infected with COVID-19: A descriptive study. Arch Gerontol Geriatr. 2020;89:104058.

4. Luo E, Zhang D, Luo H, Liu B, Zhao K, Zhao Y, et al. Treatment efficacy analysis of traditional Chinese medicine for novel coronavirus pneumonia (COVID19): an empirical study from Wuhan, Hubei Province, China. Chin Med. 2020;15:34.

5. Chen J, Wang YK, Gao Y, Hu LS, Yang JW, Wang JR, et al. Protection against COVID-19 injury by qingfei paidu decoction via anti-viral, anti-inflammatory activity and metabolic programming. Biomed Pharmacother. 2020;129:110281.

6. Li H, Lu WL, Sun YN, Xiao Y, Yang M, Yang HJ, et al. Real world clinical study of Chinese medicine treatment of 749 patients with coronavirus disease 2019. Chinese Journal of Traditional Chinese Medicine. 2020;35:3194-3198.

7. Ling CQ. Traditional Chinese medicine is a resource for drug discovery against 2019 novel coronavirus (SARS-CoV-2). J Integr Med. 2020;18(2):87-88.

8. Zhang Y, Xie H, Li Y, Li T, Yuan H, Fu X, et al. Qingfei Paidu decoction for treating COVID-19: A protocol for a meta-analysis and systematic review of randomized controlled trials. Medicine (Baltimore). 2020;99:e22040.

9. Ni L, Chen L, Huang X, Han C, Xu J, Zhang H, et al. Combating COVID-19 with integrated traditional Chinese and Western medicine in China. Acta Pharm Sin B. 2020;10:1149-1162.

10. Meng JH, He Y, Chen Q, Gao Q, Chen YG, An Jing. A retrospective study of Qingfei Paidu Decoction in the treatment of common/severe new coronavirus pneumonia. Chinese Journal of Hospital Pharmacy. 2020; 40(20):2152-2157.

11. Yang R, Liu H, Bai C, Wang Y, Zhang X, Guo R, et al. Chemical composition and pharmacological mechanism of Qingfei Paidu Decoction and Ma Xing Shi Gan Decoction against Coronavirus Disease 2019 (COVID-19): In silico and experimental study. Pharmacol Res. 2020;157:104820.

12. Shi NN, Liu B, Liang N, Ma Y, Ge YW, Yi HG, et al. Association between early treatment with Qingfei Paidu decoction and favorable clinical outcomes in patients with COVID-19: A retrospective multicenter cohort study. Pharmacological Research, 2020; 161:105290.

13. Ge GB. Deciphering the metabolic fates of herbal constituents and the interactions of herbs with human metabolic system. 2019; 17:801-802.

14. Zhou QH, Zhu YD, Zhang F, Song YQ, Jia SN, Zhu L, et al. Interactions of drug-metabolizing enzymes with the Chinese herb Psoraleae Fructus. Chin J Nat Med. 2019;17:858-70.

15. Li CH. Analysis of Qingfei Paidu Decoction in the treatment of new coronavirus pneumonia. Chinese Folk Therapy, 2020;28: 6-8.

16. Chen J, Wang YK, Gao Y, Hu LS, Yang JW, Wang JR, et al. Protection against COVID-19 injury by qingfei paidu decoction via anti-viral, anti-inflammatory activity and metabolic programming. Biomed Pharmacother. 2020;129:110281.

17. Zhao J, Tian SS, Lu D, Yang J, Zeng HW, Zhang F, et al. Systems pharmacological study illustrates the immune regulation, anti-infection, antiinflammation, and multi-organ protection mechanism of Qing-Fei-Pai-Du decoction in the treatment of COVID-19. Phytomedicine. 2020;9:153315. 
18. Fang SQ, Huang J, Zhang F, Ni HM, Chen QL, Zhu JR, et al. Pharmacokinetic interaction between a Chinese herbal formula Huosu Yangwei oral liquid and apatinib in vitro and in vivo. J Pharm Pharmacol. 2020;72:979-989.

19. Zhang F, Huang J, He RJ, Wang L, Huo PC, Guan XQ, et al. Herb-drug interaction between Styrax and warfarin: Molecular basis and mechanism. Phytomedicine. 2020;77:153287.

20. Chen PY, Yu JW,Lu FL, Lin MC, Cheng HF. Differentiating parts of Cinnamomum cassia using LC-qTOF-MS in conjunction with principal component analysis. Biomedical Chromatography, 2016; 30: 1449-57.

21. Zhang SS, Wu DD, Li H, Zhu JH, Hu WP, Lu MH, et al. Rapid identification of a-glucosidase inhibitors from Dioscorea opposita Thunb peel extract by enzyme functionalized Fe3 04 magnetic nanoparticles coupled with HPLC-MS/MS. Food \& Function, 2017; 8:3219-3227.

22. Ren T, Wang Y, Wang C, Zhang M, Huang W, Jiang J, et al. Isolation and identification of human metabolites from a novel anti-tumor candidate drug 5chlorogenic acid injection by HPLC-HRMS/MSn and HPLC-SPE-NMR. Analytical and Bioanalytical Chemistry, 2017; 409:7035-7048

23. Zhang Z , Jiang MY, Wei XY, Shi JF, Geng Z, Yang SS, et al. Rapid discovery of chemical constituents and absorbed components in rat serum after oral administration of Fuzi-Lizhong pill based on high-throughput HPLC-Q-TOF/MS analysis. Chinese Medicine, 2019; 14:6

24. Chen LL, Chen CH, Zhang XX, Wang Y, Wang SF. Identification of constituents in Gui-Zhi-Jia-Ge-Gen-Tang by LC-IT-MS combined with LC-Q-TOF-MS and elucidation of their metabolic networks in rat plasma after oral administration. Chinese Journal of Natural Medicines, 2019; 17(11): 803-821.

25. Miyagoshi M , Amagaya S, Ogihara Y. Antitussive effects of L-ephedrine, amygdalin, and Makyokansekito (Chinese traditional medicine) using a cough model induced by sulfur dioxide gas in mice. Planta Med, 1986;52(4):275-278.

26. Wu Z, Kong X, Zhang T, Ye J, Fang ZQ,Yang XJ. Pseudoephedrine/ephedrine shows potent anti-inflammatory activity against TNF-a-mediated acute liver failure induced by lipopolysaccharide/d-galactosamine. Eur J Pharmacol, 2014;724:112-121.

27. Paoletti I, Gregorio V, Baroni A, Tufano MA, Donnarumma G, Perez JJ. Amygdalin analogues inhibit IFN-ץ signalling and reduce the inflammatory response in human epidermal keratinocytes. Inflammation, 2013;36(6):1316-1326.

28. Fiebich B L, Collado J A, Stratz C, Valina C, Hochholzer W, Muoz E, et al. Pseudoephedrine inhibits T-cell activation by targeting NF-KB, NFAT and AP-1 signaling pathways. Immunopharm Immunot, 2011;34(1):98-106.

29. Deng JG, Li CY, Wang HL, Hao E, Wang Y. Amygdalin mediates relieved atherosclerosis in apolipoprotein E deficient mice through the induction of regulatory T cells. Biochem Biophys Res Commun, 2011;411(3):523-529.

30. Zhang A, Pan W, Lv J, Wu H. Protective effect of amygdalin on LPS-induced acute lung injury by inhibiting NF-KB and NLRP3 signaling pathways. Inflammation, 2017; 40(3):745-751.

31. Kant N M, Agrawal A S, Sudeshna B, Shaon N, Rahul B, Saikat C, et et al. Antiviral activity of baicalin against influenza virus H1N1-pdm09 is due to modulation of NS1-mediated cellular innate immune responses. J Antimicrob Chemother, 2014; 69(5):1298-1310.

32. Su HX, Yao S, Zhao WF, Li MJ, Xu YC. Anti-SARS-CoV-2 activities in vitro of Shuanghuanglian preparations and bioactive ingredients. Acta Pharmacologica Sinica, 2020; 41: 1167-1177

33. Wu C R, Liu Y, Yang Y Y, Zhang P, Zhong W, Wang YL, et al. Analysis of therapeutic targets for SARS-CoV-2 and discovery of potential drugs by computational methods. Acta Pharm Sin B, 2020; 10(5):766-788.

34. Yu J Y, Ha J Y, Kim K M, Young-Suk J, Jae-Chul J, Seikwan O. Anti-inflammatory activities of licorice extract and its active compounds, glycyrrhizic acid, liquiritin and liquiritigenin, in BV2 cells and mice liver. Molecules, 2015; 20(7):13041-13054.

35. Zhang Y, Zhang L, Zhang Y, Xu JJ, Sun LL, Li SZ. The protective role of liquiritin in high fructose-induced myocardial fibrosis via inhibiting NF-kB and MAPK signaling pathway. Biomed Pharmacother, 2016; 84:1337-1349.

36. Luoliu P D , Li J . Pharmacologic perspective: glycyrrhizin may be an efficacious therapeutic agent for COVID-19. International Journal of Antimicrobial Agents, 2020; 55(6):105995.

37. Bailly C , Gérard Vergoten. Glycyrrhizin: An alternative drug for the treatment of COVID-19 infection and the associated respiratory syndrome?. Pharmacology and Therapeutics, 2020, 214:107618.

38. Murck H. Symptomatic Protective Action of Glycyrrhizin (Licorice) in COVID-19 Infection?. Frontiers in Immunology, 2020; 11:1239.

39. Huang Y, Zheng SL, Xu ZS, Hou X. Effects of Alismatis rhizome on rat cytochrome P450 enzymes. Pharmaceutical Biology, $2014 ; 52(6): 681-687$.

40. Arnold C, Markovic M, Blossey K, Wallukat G, Fischer R, Dechend R, et al. Arachidonic acid-metabolizing cytochrome P450 enzymes are targets of \{omega\}-3 fatty acids. J Biol Chem. 2020;285:32720-33.

41. Kroetz DL, Zeldin DC. Cytochrome P450 pathways of arachidonic acid metabolism. Curr Opin Lipidol. 2020;13:273-83.

42. Tallima H, Ridi R. Arachidonic acid: Physiological roles and potential health benefits-A review. J Adv Res. 2017;11:33-41.

43. Lee W, Ku SK, Bae JS. Antiplatelet, anticoagulant, and profibrinolytic activities of baicalin. Arch. Pharm. Res. 2015; 38, 893-903

44. Kruse Jan Matthias, Magomedov Abakar, Kurreck Annika, et al. Thromboembolic complications in critically ill COVID-19 patients are associated with impaired fibrinolysis. Crit Care, 2020; 24: 676.

45. Cremer S, Jakob C, Berkowitsch A, Borgmann S, Pilgram L, Tometten L, et al. Elevated markers of thrombo-inflammatory activation predict outcome in patients with cardiovascular comorbidities and COVID-19 disease: insights from the LEOSS registry. Clin Res Cardiol. 2020;19:1-12.

46. Casale M, Dattilo G, Imbalzano E, Gigliotti De Fazio M, Morabito C, Mezzetti M, et al. The thromboembolism in COVID-19: the unsolved problem. Panminerva Med. 2020;16.

\section{Figures}



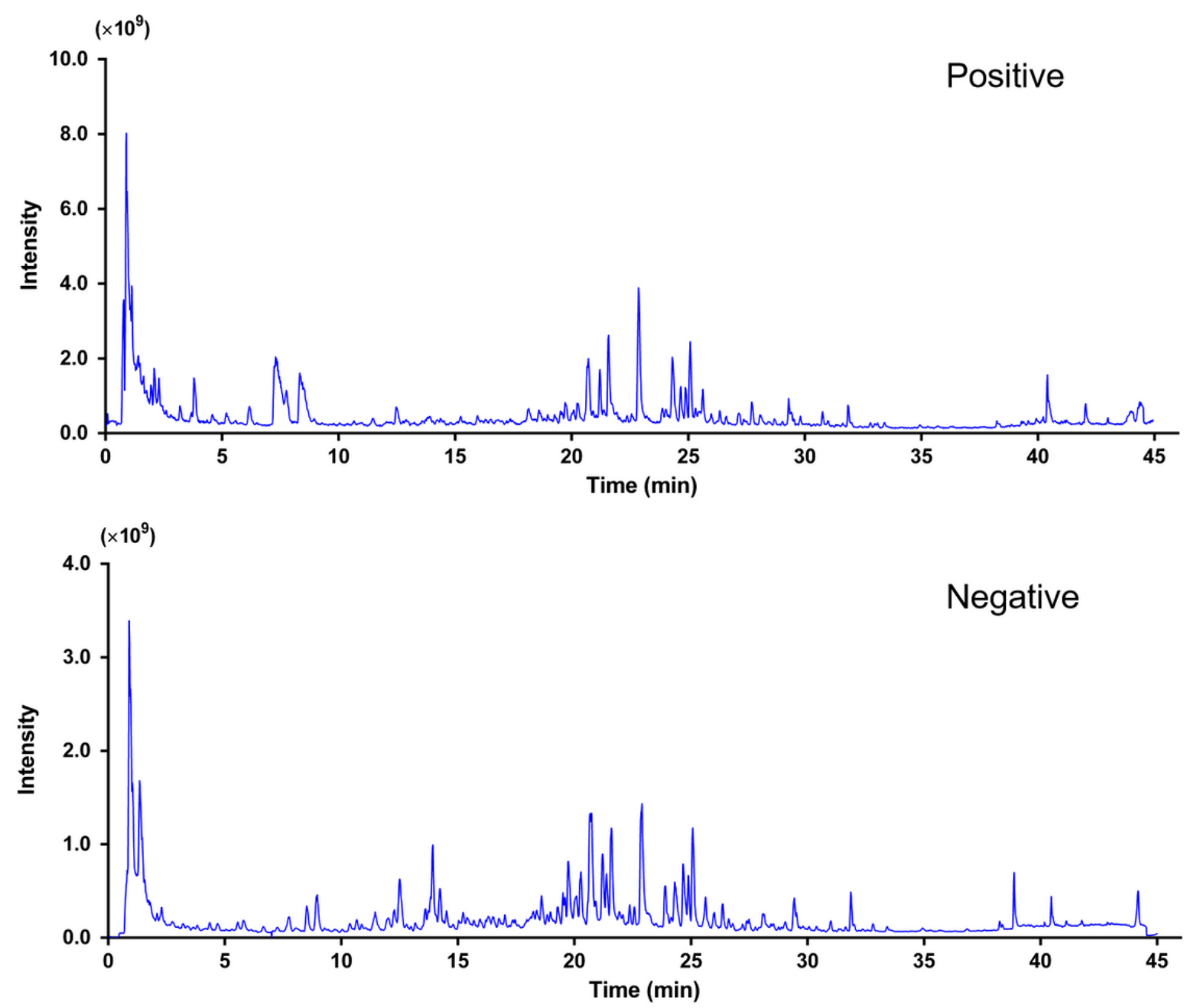

Figure 1

The total ion chromatograms (TICs) of the extract of Qingfei Paidu decoction by UHPLC-Q-Exactive Orbitrap HRMS 

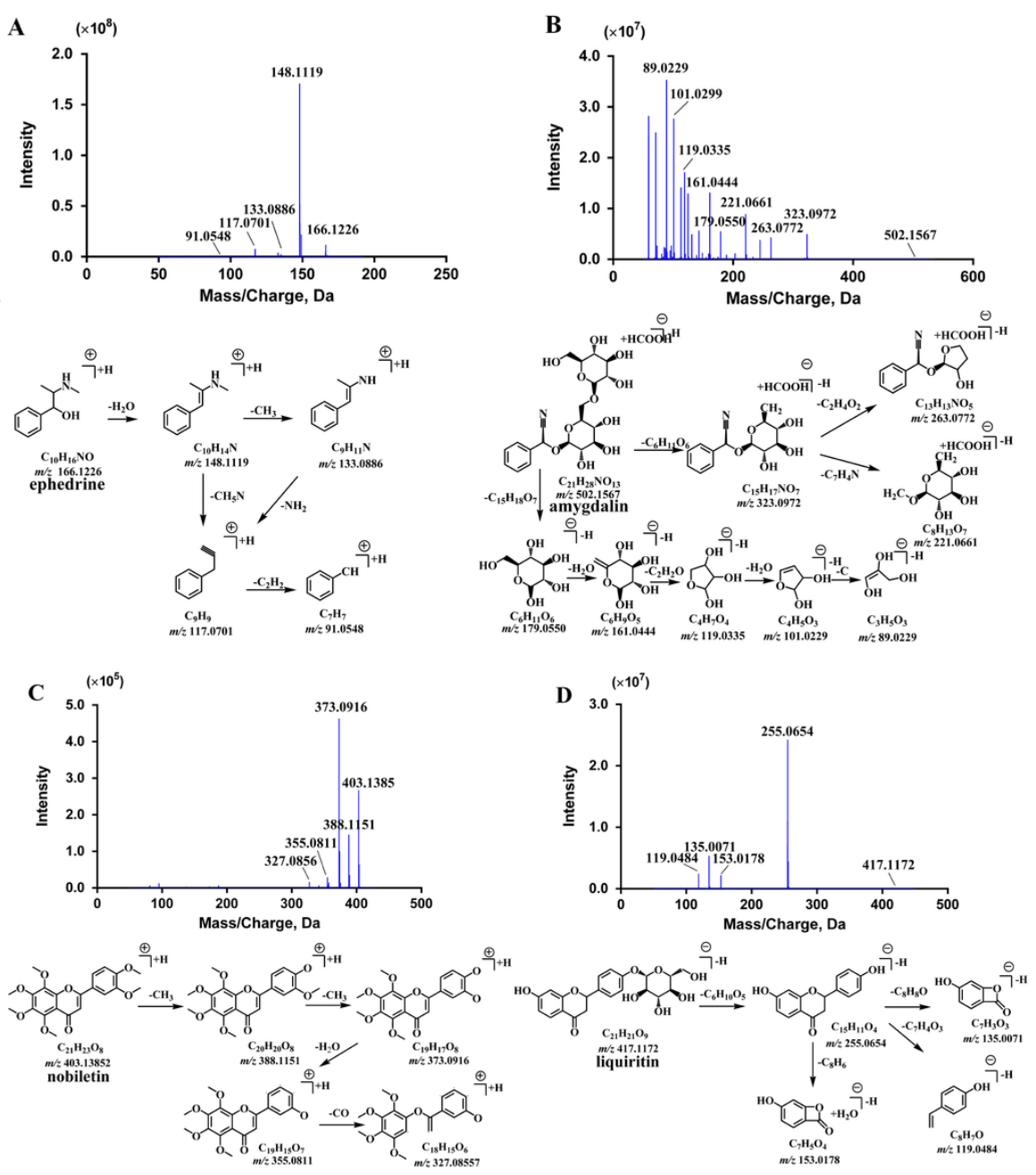

\section{Figure 2}

The mass fragment and fragmentation pathway of ephedrine (A), amygdalin (B), nobiletin (C) and liquiritin (D) 

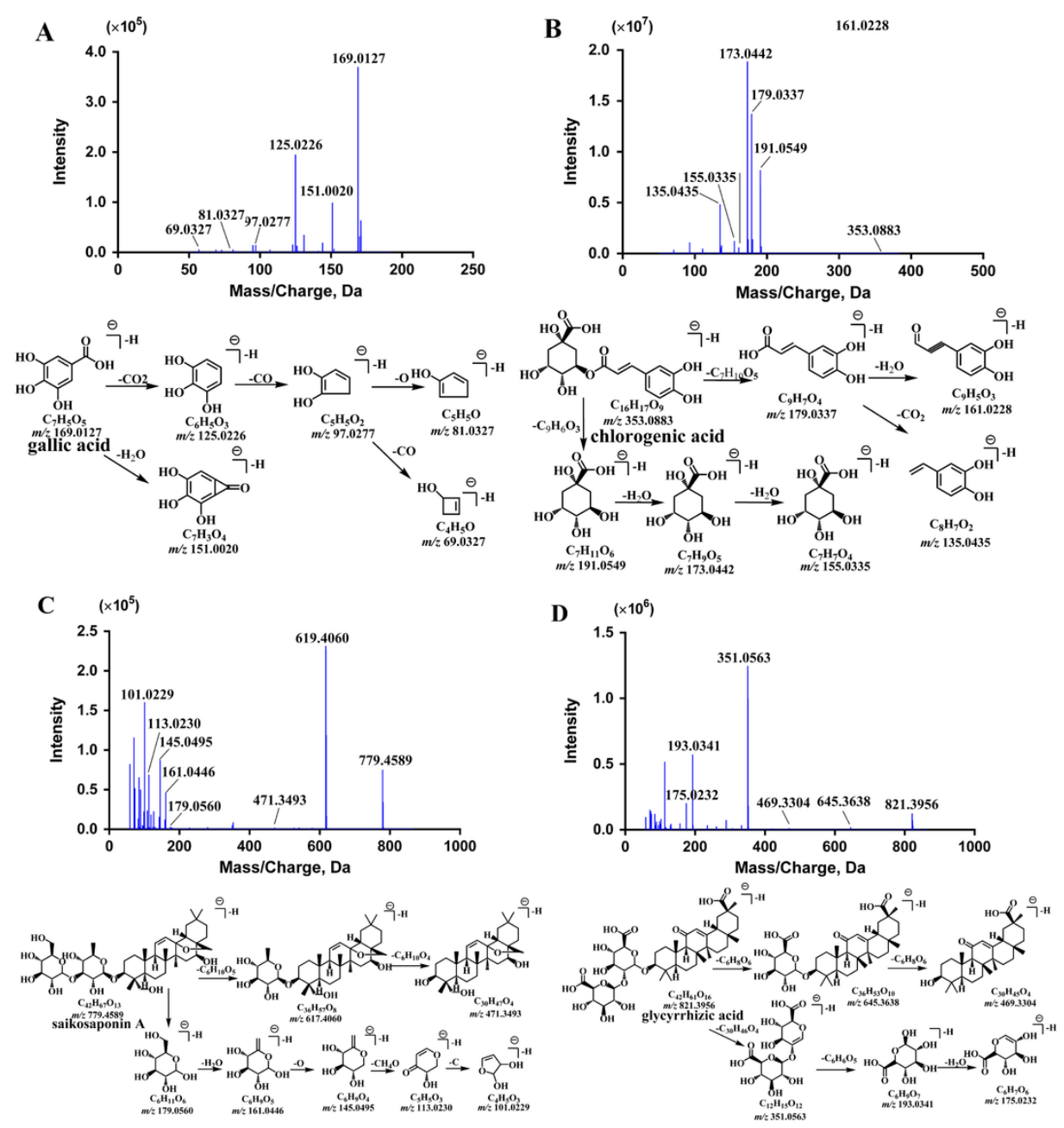

\section{Figure 3}

The mass fragment and fragmentation pathway of gallic acid (A), chlorogenic acid (B), saikosaponin A (C) and glycyrrhizic acid (D) 展

Figure 4

Structures of the absorbed prototype compounds (alkaloids and flavonoids) in mice serum after oral administration of Qingfei Paidu decoction 层

\section{Figure 5}

Structures of the absorbed prototype compounds (organic acids, triterpene saponins, and others) in mice serum after oral administration of Qingfei Paidu decoction 

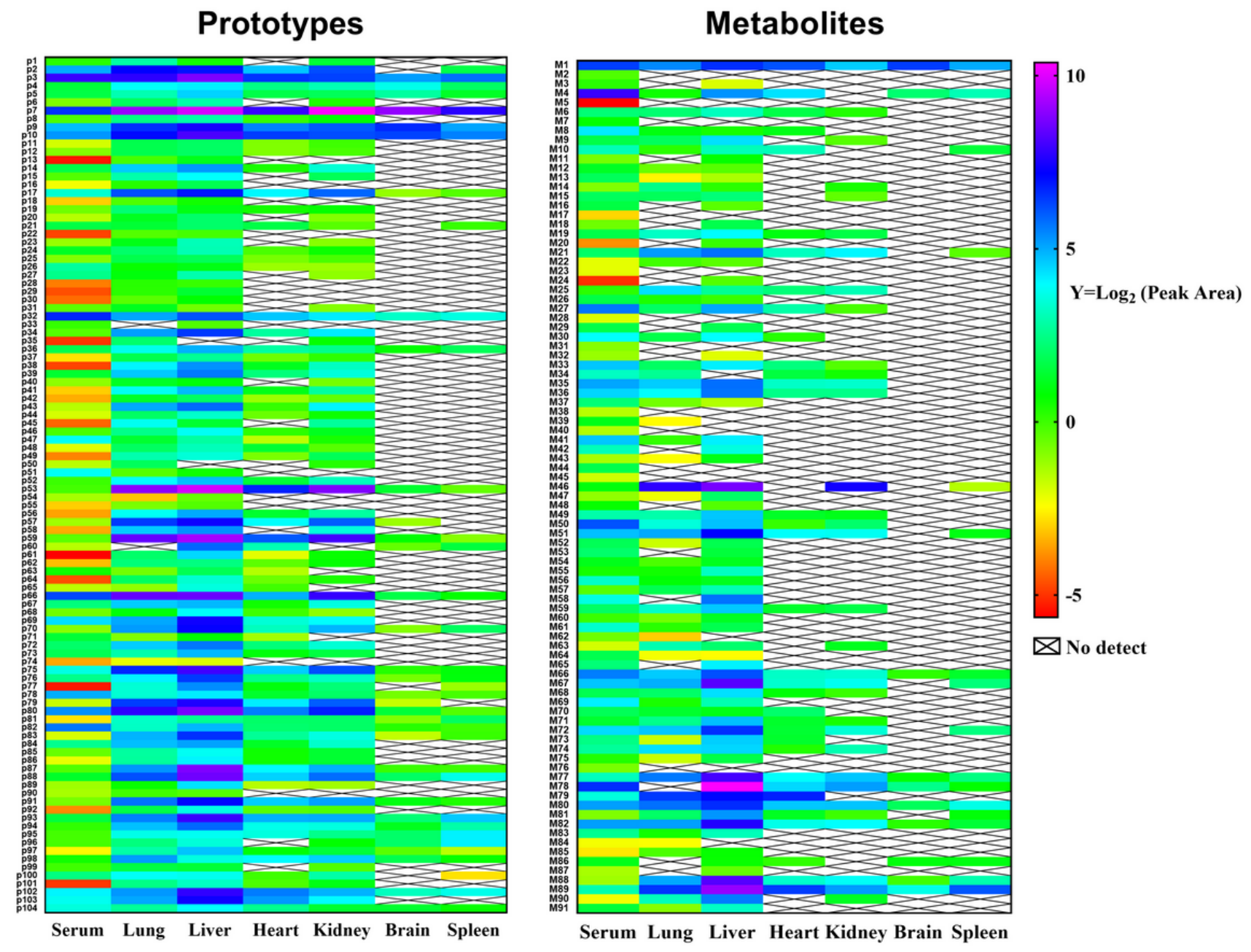

Figure 6

The heat map of the peak areas of absorbed components and metabolites in mice serum and tissues after oral administration Qingfei Paidu decoction 


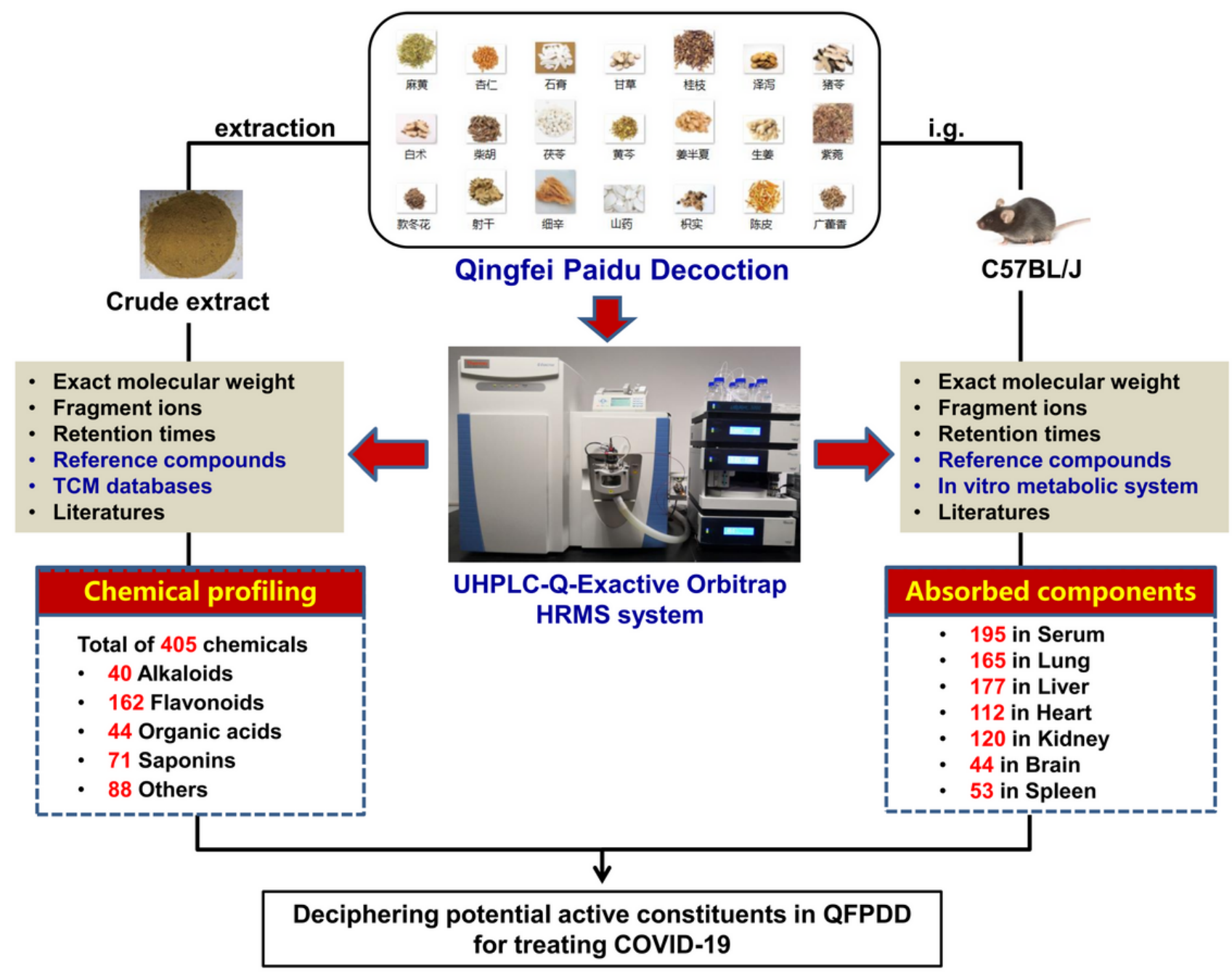

Figure 7

Research strategy for clarifying the chemical composition and absorption components of Qingfei Paidu decoction based on UHPLC-Q-Exactive Orbitrap HRMS technology 